FEDERAL

RESERVE

BANK of

ST. LOUIS

\section{RESEARCH DIVISION} Working Paper Series

\title{
The Analytics of Technology News Shocks
}

\author{
Bill Dupor \\ and \\ M. Saif Mehkari \\ Working Paper 2013-036A \\ https://doi.org/10.20955/wp.2013.036
}

November 2013

FEDERAL RESERVE BANK OF ST. LOUIS

Research Division

P.O. Box 442

St. Louis, MO 63166

The views expressed are those of the individual authors and do not necessarily reflect official positions of the Federal Reserve Bank of St. Louis, the Federal Reserve System, or the Board of Governors.

Federal Reserve Bank of St. Louis Working Papers are preliminary materials circulated to stimulate discussion and critical comment. References in publications to Federal Reserve Bank of St. Louis Working Papers (other than an acknowledgment that the writer has had access to unpublished material) should be cleared with the author or authors. 


\title{
The Analytics of Technology News Shocks*
}

\author{
Bill Dupor ${ }^{\dagger}$ and M. Saif Mehkari ${ }^{\ddagger}$
}

November 2013

\begin{abstract}
This paper constructs several models in which, unlike the standard neoclassical growth model, positive news about future technology generates an increase in current consumption, hours and investment. These models are said to exhibit procyclical news shocks. We find that all models that exhibit procyclical news shocks in our paper have two commonalities. There are mechanisms to ensure that: (I) consumption does not crowd out investment, or vice versa; (II) the benefit of forgoing leisure in response to news shocks outweighs the cost. Among the models we consider, we believe, one model holds the greatest potential for explaining procyclical news shocks. Its critical assumption is that news of the future technology also illuminates the nature of this technology. This illumination in turn permits economic actors to invest in capital that is forward-compatible, i.e. adapted to the new technology. On the technical side, our paper reintroduces the Laplace transform as a tool for studying dynamic economies analytically. Using Laplace transforms we are able to study and prove results about the full dynamics of the model in response to news shocks.
\end{abstract}

\footnotetext{
${ }^{*}$ The authors would like to thank Paul Evans, Jang Ting Guo, Jing Han, Alok Johri, Aubhik Khan, Nan Li, Masao Ogaki, Hammad Qureshi, Julia Thomas, Yi-Chan Tsai, and conference and seminar participants at the Federal Reserve Bank of St. Louis, the University of Western Ontario and the Midwest Macroeconomics Meetings. The analysis and conclusions set forth do not reflect the views of the Federal Reserve Bank of St. Louis, the Federal Reserve System or the Board of Governors.

${ }^{\dagger}$ Research Division, Federal Reserve Bank of St. Louis, USA. Email: william.d.dupor@stls.frb.org; billdupor@gmail.com

${ }^{\ddagger}$ Department of Economics, University of Richmond, USA. Email: smehkari@richmond.edu
} 


\section{Introduction}

The optimal response of aggregate consumption, investment and hours in the neoclassical growth model to an unanticipated permanent (or near permanent) technology increase is well-understood. For most specifications used by researchers, all three variables increase. ${ }^{1}$ A technology improvement increases capital's efficiency; thus, the desired capital stock increases. The increase in the actual capital stock twoards its desired level is achieved by greater investment. Importantly, greater investment need not come at the cost of a drop in consumption. Rather, since the technology improvement shifts out the production frontier immediately, creating additional consumption and investment is feasible. Moreover, an hours increase is optimal because a higher marginal product of labor induces a substitution effect away from leisure that outweighs the wealth effect, which pushes in the opposite direction.

Next, consider the standard growth model's response to news of a future technology increase. The responses of these variables and the incentives that drive these responses are different. In the standard model, all three variables will not increase. Typically, labor falls upon the arrival of the news. The above-described wealth effect on leisure is operative; however, there is no offsetting substitution effect because the technology increase has not materialized immediately.

With a labor decline, the only way consumption can increase in response to the news is if investment falls. An investment decline is optimal because there is incentive to delay building additional capital stock until technology actually increases. Thus, in the standard model, positive news about future technology can cause a decline in labor and investment, and an increase in consumption (see Figure 1)..$^{2,3}$

This paper studies variants on the standard model that are capable of generating procyclical responses. Each model has mechanism(s) to ensure that: (I) consumption does not

\footnotetext{
${ }^{1}$ Campbell (1994) establishes this by simulation using several functional forms of preferences and model parameterizations. He does provide cases where, when preferences are non-separable in consumption and labor, that consumption declines in response to technology shocks.

${ }^{2}$ An alternative, but equaling puzzling, response to good news about future technology is that labor hours increase, while consumption declines. This occurs for a small region of growth model's parameter space.

${ }^{3}$ There is some support for procyclical news shocks in U.S. data. Systematic empirical work supporting the news shock explanation includes Schmitt-Grohe and Uribe (2012) and Beaudry and Portier (2006). The former estimate a business cycle model with news (anticipated) and current (unanticipated) shocks and find that news shocks explain a greater fraction of output volatility than current shocks. The latter estimate that the component of innovations to stock prices, not correlated with current productivity, is correlated with expected future productivity. Barsky and Sims (2009), using a different identification scheme, deliver an opposite result (i.e. news shocks are not procyclical). Other relevant empirical research supporting this explanation includes Beaudry, Dupaigne,and Portier (2008), Beaudry and Lucke (2010), and Khan and Tsoukalas (2012) as well as Leeper, Walker and Yang (2012).
} 
crowd out investment, or vice versa; (II) the return to forgoing leisure is sufficiently high.

In our first model, we modify the neoclassical production function to have a convex production frontier between consumption and investment, i.e. production complementarity. In the standard model, the marginal rate of transformation between consumption and investment is fixed at one. Here, this marginal rate of transformation depends upon the consumption-investment ratio. We provide both sufficient and necessary conditions for the model to exhibit procyclical technology news shocks. These conditions depend upon the values of the model's underlying parameters.

With a convex production frontier, greater consumption (investment) increases the marginal product of labor towards the production of investment (consumption). This effect tends to increase investment and labor upon the arrival of the news if there is a consumption boom. ${ }^{4}$ This achieves Condition I: consumption does not crowd out investment.

Consumption-investment complementarity causes the two variables to comove; however, it is possible that the two variables might fall rather than increase in response to positive news. In this case, the planner takes leisure over consumption (and capital accumulation) in the short run. This arises if there is too much curvature in the utility function because, in this case, the intertemporal smoothing motive for leisure becomes too strong. As such, there must be sufficiently low curvature in order that hours, consumption and investment comove procyclically in response to positive news. Restricting the curvature to be low ensures our Condition II, that the relative benefit of forgoing leisure is sufficiently strong.

Our second model contains a preference-based mechanism for generating procyclical technology news shocks. ${ }^{5}$ Here, we assume a preference externality such that the marginal disutility of own hours worked is falling in the economy-wide average hours worked. ${ }^{6}$ The preference externality acts, from each household's perspective, as a preference shock that expands each household's willingness to supply labor. This endogenous labor supply mechanism directly decreases the household utility cost of forgoing leisure (satisfying Condition II) and the increase in labor expands output sufficiently for both consumption and investment to increase (satisfying Condition I).

After proving a theorem for each model to exhibit procyclical news shocks, we conduct a quantitative analysis. Here, we find a drawback with both models. Each generates quan-

\footnotetext{
${ }^{4}$ In an extreme but illustrative case, if consumption and investment are produced in a Leontief manner, then consumption and investment comove perfectly.

${ }^{5}$ That is, the production side of this second model is neoclassical.

${ }^{6}$ Despite being non-standard, the preferences are consistent with balanced growth and both consumption and leisure are normal goods from each household's perspective.
} 
titatively small consumption booms in response to positive news. Consumption is nearly acyclical.

As a result of the near acyclicality of consumption, we develop two distinct extensions of the production complementarities model. First, we replace balanced-growth preferences with GHH preferences. Second, we add investment adjustment costs to the model. With either of these additions we are able to generate quantitatively larger consumption booms and also support procyclical news shocks with greater curvature in the utility function.

In our view, the greatest promise for explaining the phenomenon is a situation where Condition II, i.e. a strong return to forgoing leisure, is achieved because there is a benefit to starting investing early beyond that inherent in the basic neoclassical model. In our view, it is more plausible that this benefit is production-based rather than preference-based. To this end, we construct the final model of the paper, which introduces the concept of "forward-compatible investment."

The starting point for the forward-compatibility model is a neoclassical economy with investment-specific technology (IST) shocks and production complementarity. In addition, we assume investment made between the news arrival and the actual IST increase is partially forward-compatible; investment can take partial advantage of future technology improvements. This causes an investment boom upon the arrival of the news as the social planner builds capital in anticipation of the IST increase, even though there is no immediate technological improvement. This "preparatory-phase" investment is optimal because it allows the planner to smooth consumption while accumulating capital towards the eventual higher steady state.

It is beneficial, in our view, for a modification of the neoclassical model to be as minimal as possible. This makes it less likely that the resulting model does violence to the existing theory of unanticipated shocks. To this end, we note that adding forward compatibility of investment is not a significant departure from the neoclassical model along two key dimensions. First, our solution adds no new state variables to the neoclassical model. This is useful because the lack of any new state variables allows for a thorough examination of the mechanism in a two dimensional space; each additional state variable would add two more dimensions to the model (a state and a co-state). Second, our model collapses to the standard neoclassical model with production complementarities when the exogenous driving process is a contemporaneous shock. That is, the forward compatibility does not operate when the business cycle is driven by contemporaneous shocks. This allows our model to be directly comparable to the basic neoclassical model. 
To allow for a complete theoretical analysis, we use a continuous time model. A continuous time framework allows us to use the method of Laplace transforms. The Laplace transform is useful for studying linear differential equations with constant coefficients and exogenous (non-homogeneous) terms with discontinuities. ${ }^{7}$ Once we log-linearize the growth model, our differential equations take exactly this form. The discontinuity in our model is present because of the forecastable jump in future technology.

There are several existing papers on news-driven cycles in dynamic general equilibrium models. Beaudry and Portier (2007) study the difficulty that the neoclassical model has in exhibiting procyclical news shocks. They provide necessary condition on production sets for news shocks to create consumption and investment comovement. Importantly, they observe that many production technologies used in macro do not satisfy this necessary condition. Also, they calibrate a model with one feature capable of generating news-driven cycles: production complementarities of the kind studied in our paper. Their theoretical work does not explore the analytics underlying the dynamics of news-driven cycles. Beaudry and Portier (2004) generate news-driven cycles by modeling final consumption as a function of non-durables and the capital stock. Jaimovich and Rebelo (2009) generate large responses to news shocks, by adding variable capital utilization and two dynamic state variables to the neoclassical model: lagged investment through adjustment costs and time non-separable preferences. Christiano et. al. (2007) use investment adjustment costs and habit persistence to generate news-driven business cycles. Wang (2012) analyzes and compares three existing models generating procyclical news shocks via a labor market diagram. This graphical analysis is very useful for understanding the static relationships in these models, but not as useful for understanding the models' dynamics. In the same paper, Wang develops a model where an endogenous markup resolves this comovement puzzle.

Nah (2009) uses production complementarities and financial frictions to support procyclical news shocks. Gunn and Johri (2011) and Qureshi (2009) each develop a learning-by-doing model. In response to news about future technological improvement, forward-looking agents increase hours worked and investment immediately in order to build up their stock of knowledge. This amplifies the benefit of the future technology increase. Gunn and Johri (2009) show that learning-by-doing combined with variable capital utilization can generate procyclical stock prices. Qureshi (2009) shows that learning-by-doing along with an intratemporal adjustment cost can generate sectoral comovement in response to news about neutral and

\footnotetext{
${ }^{7}$ Several introductory textbooks on differential equations describe the $\mathrm{L}$ aplace transform, including Boyce and DiPrima (1969) and Tenenbaum and Pollard (1985). Early applications of the transform to economics include Judd $(1982,1985)$.
} 
sector-specific technologies. Comin, Gertler and Santacreu (2009) develop a model with shocks to the number of new ideas capable of increasing the efficiency of capital and labor. However, resources must be allocated to transform ideas into actual technologies. In their model, news is the arrival of new ideas; whereas in our model, news is (in part) the arrival of useful information about the nature of future technologies. Tsai (2009) uses variable capital utilization and preferences designed to minimize wealth effects on labor supply, along with fixed costs to adopt new vintages of capital. The latter feature in his model has a feel very similar to the forward-compatibility assumption in Section 5 of our paper.

Our paper differs from the above numerical/simulation-based results, along with the theoretical results in Beaudry and Portier (2007), in that, to the best of our knowledge, it is the first paper to study the full dynamics of news shocks analytically. This allows us to shed light on how news shocks in general work.

In the next section, we describe the production complementarity model, characterize its optimal allocation and provide conditions under which the model supports procyclical technology news shocks. In Section 3, we do the analogous examination of a preference-based mechanism capable of supporting these type of news shocks. Section 4 analyzes quantitative and calibration issues with the baseline production complementary model, and develops modifications to address these issues. Section 5 studies a model of forward-compatible investment, and Section 6 concludes.

\section{Procyclical News Shocks via Production Comple- mentarity}

Consider the following variant of the neoclassical growth model.

\section{The Model}

Consumption, $C(t)$, and investment, $I(t)$ are produced according to:

$$
F[C(t), I(t)]=K(t)^{\alpha}(A(t) N(t))^{1-\alpha}
$$

where $K(t)$ and $N(t)$ represent capital and hours respectively. Assume

$$
F(C, I)=\left[\theta C^{v}+(1-\theta) I^{v}\right]^{1 / v}
$$

where $\alpha, \theta \in(0,1), t \in[0, \infty]$ and $v \geq 1$. 
Our sole departure from the neoclassical model pertains to the definition of $F(C, I)$, which represents the production possibility frontier for consumption and investment given the amount of inputs. We allow for the possibility of complementarities between the production of consumption and investment goods. If $v=1$, the equation collapses to the standard model. As $v$ increases, the complementarity between the production of the two goods increases. If $v=\infty$, the production frontier takes a Leontief form. Figure 2 shows how the complementarity between consumption and investment changes as $v$ changes.

We can interpret $v$ as measuring the factor substitutability between the consumption and investment sectors of a more general model. In the basic neoclassical model $(v=1)$, factors are equally productive in both the consumption and investment sectors. As a result, the relative price of consumption to investment remains constant irrespective of how much resources are being devoted to producing consumption versus investment. In our model, factors are not equally productive in both sectors. As $v$ increases, a factor productive in one sector is less and less productive in the other sector. For example, a worker that produces goods in the consumption sector, when moved to the investment sector will become less productive. $^{8}$

The law of motion of capital is:

$$
\dot{K}(t)=I(t)-\delta K(t)
$$

where $\delta$ is the capital depreciation rate.

A social planner ranks utility over different consumption and hours time paths using:

$$
U=(1-\sigma)^{-1} \int_{0}^{\infty} e^{-\rho t}[C(t) \exp (-N(t))]^{1-\sigma} d t
$$

where $\sigma \geq 0$ is the curvature parameter in the utility function and $\rho>0$ is the discount rate. $^{9}$

Next, a positive technology news shock is an increase in technology arriving at time $T$

\footnotetext{
${ }^{8}$ An alternative mechanism to the bowed-out production frontier in equation (1) is intersectoral adjustment costs. Suppose that in the planner problem we replace $F(C, I)$ with

$$
F^{A D J}(C, I) \equiv(C+I)\left[1+\frac{\psi_{Y}}{2}\left(\frac{1}{\theta} \frac{C}{I}-1\right)^{2}\right]
$$

Given the above form, the intersectoral adjustment cost and production complementarity models are isomorphic up to the log-linearization.

${ }^{9}$ These preferences exhibit balanced growth. Holding fixed hours, $\sigma$ is the inverse of the intertemporal elasticity of substitution for consumption.
} 
that becomes anticipated at time zero. Thus, at time zero, the perfect foresight time path for technology becomes:

$$
A(t)=\left\{\begin{array}{ccrl}
\bar{A} & \text { for } t & \in[0, T) \\
\bar{A}=\bar{A}+\epsilon & t \geq T
\end{array}\right.
$$

where $\bar{A}$ denotes the initial steady-state technology level. A contemporaneous (or unanticipated) technology shock corresponds to the case when $T=0$.

It is useful to define the following

Definition 1. A model exhibits procyclical technology news shocks if an anticipated increase in future technology (i.e. a positive technology news shock) leads to an increase in current consumption, investment and hours.

Because we only study technology shocks in this paper, we will often omit the word 'technology' when referring to technology news shocks.

\section{The Planning Problem and Its Solution}

The social planner chooses $C, I, K$ and $N$ to maximize $U$ subject to (1), (2) and (3), taking as given the initial condition $K(0)$ and time path of technology given by (5).

The current value Hamiltonian associated with the problem is:

$$
H=(1-\sigma)^{-1} C^{1-\sigma} \exp [-(1-\sigma) N]+\Lambda(I-\delta K)+\Phi\left(K^{\alpha}(A N)^{1-\alpha}-F(C, I)\right)
$$

The first-order necessary conditions at an interior solution satisfy the following:

$$
\begin{gathered}
-\frac{U_{N}}{U_{C}}=(1-\alpha) \frac{F}{N}\left(F_{C}\right)^{-1} \\
\frac{U_{C}}{\Lambda}=\frac{F_{C}}{F_{I}} \\
\frac{\dot{\Lambda}}{\Lambda}-\rho=\delta-\alpha \frac{F}{K}\left(F_{I}\right)^{-1}
\end{gathered}
$$

along with an initial condition on capital and a transversality condition.

Equation (6) is the intratemporal Euler equation between consumption and labor hours, equation (7) is the intratemporal Euler equation between consumption and investment, and equation (8) is the optimal capital accumulation equation. All of these equations are similar to their neoclassic counterparts. The sole difference is that $F_{C}=F_{I}=1$ in the basic 
neoclassical model. With production complementarities $F_{C}$ and $F_{I}$ change with level of consumption and investment.

Log-linearizing these equations, ${ }^{10}$ we have the following three optimality conditions:

$$
\begin{gathered}
n=v s_{I}(i-c) \\
(v-1)(i-c)=\lambda-(-\sigma c-z n) \\
\dot{\lambda}=-(\rho+\delta)\left[v\left(1-s_{I}\right)(c-i)+i-k\right]
\end{gathered}
$$

where $z=(1-\sigma)(1-\alpha) /\left(1-s_{I}\right)$ and $s_{I}=(\alpha \delta) /(\rho+\delta)$.

Equation (9) ensures an efficient labor allocation. As consumption rises, the marginal utility of consumption falls and the planner increases leisure. An increase in investment shifts out labor supply.

Equation (10) ensures an efficient consumption-investment split. The left-hand side is the price of investment in units of consumption. Because of complementarity, investment becomes more expensive when production of consumption is relatively low. The right-hand side is the marginal utility of investment minus the marginal utility of consumption.

Equation (11) is the intertemporal consumption Euler equation. It differs from the neoclassical model in that $\lambda$ is not simply the derivative of the marginal utility of consumption. There is an additional relative price effect because of the convex production frontier.

The two resource constraints and the definition of output are given by:

$$
\begin{gathered}
\left(1-s_{I}\right) c+s_{I} i=\alpha k+(1-\alpha)(a+n) \\
\dot{k}=\delta(i-k) \\
y=\alpha k+(1-\alpha)(a+n)
\end{gathered}
$$

Equation (12) is the static resource constraint. Equation (13) is the law of motion for capital. Equation (14) gives the definition of output.

\section{News-Driven Business Cycles}

We next study under what conditions the model exhibits procyclical news shocks. We

\footnotetext{
${ }^{10}$ The system is log-linearized around the initial steady-state, which is consistent with the constant technology $\bar{a}$. A lower case letter denotes the $\log$ deviation of that variable from its upper case counterpart.
} 
subdivide our proof into first establishing the procyclicality and comovement between the variables at time zero $(t=0)$, and then the procyclicality and comovement between variables for time $t \in(0, T)$. The latter results for $t \in(0, T)$ distinguish our theoretical work from others.

Lemma 1. Suppose the economy experiences a positive technology news shock. Consumption, investment and hours will comove at time zero if and only if $v>v^{*}=(1-\alpha)^{-1}$

Proof. All proofs are contained in Appendix A.

Lemma 2. Suppose the economy experiences a positive technology news shock. Consumption, investment and hours will comove procyclically, with respect to the expectations of future technology, at time zero if and only if $v>v^{*}$ and $\lambda(0)>0$.

The intuition for Lemmas 1 and 2 can be understood using Figure 3. Figure 3 plots the solution to the static consumption-investment decision holding fixed the marginal utility of investment. It plots this for the cases with and without production complementarity.

Substituting out the optimal hours from the production equation (12), we have:

$$
\alpha k+(1-\alpha) a=\left(1-\phi_{I}^{P C}\right) c+\phi_{I}^{P C} i
$$

where $\phi_{I}^{P C}=(1-v(1-\alpha)) s_{I}$.

This is plotted as $L_{1}$ in Figure 3(a) and Figure 3(b). In the absence of production complementarity, this is a downward-sloping line, as seen in Figure 3(a). ${ }^{11}$ Intuitively, when consumption rises, hours cannot optimally rise because leisure is a normal good; therefore, investment must fall. With sufficiently strong complementarity, i.e. $v>(1-\alpha)^{-1}, L_{1}$ is upward sloping as seen in panel (b).

This occurs because, with strong complementarity, an increase in investment raises the marginal product of labor in producing the consumption good. This higher marginal product of labor implies that both hours and consumption can increase. An investment decline, on the other hand, will go hand-in-hand with a reduction in consumption.

Next, consider $L_{2}$, the consumption-investment Euler equation with optimal hours substituted out:

$$
\gamma_{I}^{P C} i-\left(\sigma+\gamma_{I}^{P C}\right) c=\lambda
$$

\footnotetext{
${ }^{11} \mathrm{We}$ are assuming that the economy is at its steady-state associated with $\bar{A}$ at time zero.
} 
where $\gamma_{I}^{P C}=(v-1)-\left[v(1-\alpha)(1-\sigma) s_{I}\right] /\left(1-s_{I}\right)$.

In general, the slope of $L_{2}$ can be either positive or negative. The slope depends most crucially on $\nu$. To generate procyclical news shocks, $\nu$ must be large. To understand why $L_{2}$ can be upward-sloping, consider the consumption-leisure Euler equation. For the assumed utility function, consumption equals the real wage (ignoring complementarity in production). Because the real wage is simply labor's share in production, hours are a linear function of the output-consumption ratio. Thus, if the planner decided to increase investment relative to consumption, hours worked increases. This is seen in equation (9). Note that adding production complementarity (i.e. setting $\nu>1$ ) increases the hours effect because it increases the marginal product of hours in producing the consumption good.

Next, suppose we consider an increase in the marginal utility of investment, $\lambda$, at time zero. ${ }^{12}$ First, an increase in $\lambda(0)$ does not shift $L_{1} \cdot{ }^{13}$ Second, an increase in $\lambda(0)$ induces a shift leftward of $L_{2}$ either with or without complementarity. As the marginal utility of investment increases, the social planner shifts away from consumption for a given level of investment. Even though $L_{2}$ moves in the same direction in either case, the implication for the optimal investment-consumption pair is different between the two cases. Because $L_{1}$ is downward sloping without complementarity, investment rises but consumption falls; however, $L_{1}$ is upward sloping with complementarity and both investment and consumption rise. Intuitively, the increase in investment raises the marginal product of labor towards consumption when there is production complementarity. The fall in the relative price of consumption leads the planner to increase hours worked.

Lemma 3. Suppose the economy experiences a positive technology news shock. Also, assume that $v>v^{*}$. Consumption, investment and hours will comove procyclically for all time $t<T$ if $\forall t<T, \dot{\lambda} \geq 0$ and $\dot{k} \geq 0$

Equation (15) implies that if $k$ is increasing over time, then output also increases over time holding $a$ at its steady state level of zero. As such, $\dot{k} \geq 0$ causes $L_{1}$ to progressively shift rightward, shifting out the production frontier. When $\dot{\lambda} \geq 0$, the marginal utility of investment is increasing over time. This causes the planner to shift production away from consumption into investment. This results in a leftward shift in $L_{2}$. As illustrated in Figure

\footnotetext{
${ }^{12}$ For now, we take the increase in $\lambda(0)$ as given. Later, starting with Lemma 4, we provide a condition for which time zero news of a technology increase at time $T$ results in an increase in $\lambda(0)$.

${ }^{13}$ This is because our preferences imply that labor is stationary along a balanced growth path; the consumption-leisure Euler equation, and therefore $L_{1}$ does not depend on the co-state variable.
} 
4 , these two effects cause consumption and investment to continue increasing for all time $t<T$.

We have thus far studied what happens to $c, i$ and $n$ when $\lambda(0)$ increases in response to good news. We now provide conditions on parameters under which this increase in $\lambda(0)$ obtains.

The log-linearized dynamic system is: ${ }^{14}$

$$
\left[\begin{array}{l}
\dot{\lambda}(t) \\
\dot{k}(t)
\end{array}\right]=\left[\begin{array}{cc}
\Gamma_{\lambda, \lambda}^{P C} & \Gamma_{\lambda, k}^{P C} \\
\Gamma_{k, \lambda}^{P C} & \Gamma_{k, k}^{P C}
\end{array}\right]\left[\begin{array}{c}
\lambda(t) \\
k(t)
\end{array}\right]+\left[\begin{array}{c}
b_{\lambda, a}^{P C} \\
b_{k, a}^{P C}
\end{array}\right] a(t)
$$

In the presence of a news shock, there is a discontinuous forcing term in the dynamic system. In equation (17), $a(t)$ is a step function which takes on the value zero for all time $t<T$ and a value of $\ln (1.01)$ for all time $t \geq T$, i.e. $\epsilon=.01 \bar{A}$.

Laplace transforms lend themselves nicely here. Using these transforms, we can map our problem into the frequency domain where the problem is continuous and solvable using standard techniques. Once we have solved the dynamic system in this new domain we can then map the solution back into the time-domain. The resulting time-paths of $k(t)$ and $\lambda(t)$ for our system are:

$$
\begin{aligned}
& k(t)=\left\{\begin{array}{cc}
\frac{\Gamma_{k, \lambda}^{P C} \lambda(0)}{\mu_{1}-\mu_{2}} e^{\mu_{1} t}+\frac{\Gamma_{k, \lambda}^{P C} \lambda(0)}{\mu_{2}-\mu_{1}} e^{\mu_{2} t} & \text { for } t \in[0, T) \\
\frac{\Gamma_{k, \lambda}^{P C} \lambda(0)}{\mu_{1-\mu_{2}}} e^{\mu_{1} t}+\frac{\Gamma_{k, \lambda}^{P C} b_{\lambda, a}^{P C}-\Gamma_{\lambda, \lambda}^{P C} b_{k, a}^{P C}}{\mu_{1} \mu_{2}}+\frac{\Gamma_{k, \lambda}^{P C} b_{\lambda, a}^{P C}+\left(\mu_{1}-\Gamma_{\lambda, \lambda}^{P C}\right) b_{k, a}^{P C}}{\mu_{1}\left(\mu_{1}-\mu_{2}\right)} e^{\mu_{1}(t-T)} & t \geq T
\end{array}\right. \\
& \lambda(t)=\left\{\begin{array}{cc}
\frac{\left(\mu_{1}-\Gamma_{k, k}^{P C}\right) \lambda(0)}{\mu_{1}-\mu_{2}} e^{\mu_{1} t}+\frac{\left(\mu_{2}-\Gamma_{k, k}^{P C}\right) \lambda(0)}{\mu_{2}-\mu_{1}} e^{\mu_{2} t} & \text { for } t \in[0, T) \\
\frac{\left(\mu_{1}-\Gamma_{k, k}^{P C}\right) \lambda(0)}{\mu_{1}-\mu_{2}} e^{\mu_{1} t}+\frac{\Gamma_{\lambda, k}^{P C} b_{k, a}^{P C}-\Gamma_{k, k}^{P C} b_{\lambda, a}^{P C}}{\mu_{1} \mu_{2}}+\frac{\Gamma_{\lambda, k}^{P C} b_{k, a}^{P C}+\left(\mu_{1}-\Gamma_{k, k}^{P C}\right) b_{\lambda, a}^{P C}}{\mu_{1}\left(\mu_{1}-\mu_{2}\right)} e^{\mu_{1}(t-T)} & t \geq T
\end{array}\right.
\end{aligned}
$$

where $\mu_{1}$ and $\mu_{2}$ are the eigenvalues of the $\Gamma^{P C}$ matrix. ${ }^{15}$ In Appendix $\mathrm{B}$, we prove that $\mu_{1}$ and $\mu_{2}$ are real with one being positive and the other negative. Without loss of generality, let $\mu_{1}<0$ and $\mu_{2}>0$.

The solutions for the time paths of $k$ and $\lambda$ show that the dynamics of the system before time $T$ are being determined not only by the stable eigenvalue, but also the unstable eigenvalue. This is important. Without a role for the unstable eigenvalue, the system would

\footnotetext{
${ }^{14}$ The values of $\Gamma_{.,}^{P C}$ and $b_{., a}^{P C}$ can be found in the online Appendix B.

${ }^{15}$ The online Appendix B contains the derivation of (18) and (19)
} 
be on a new stable manifold, corresponding to a higher permanent level of $a(t)$, for all $t<T$. Along the stable manifold capital and the shadow value of investment do not comove. This will result in a negative comovement between the variables for $t \in[0, T)$. After time $T$, the system is on a new stable path and the dynamics are then determined by the new stable manifold alone.

The above solution has one undetermined variable $\lambda(0)$. We seek a path for $(\lambda, k)$ that is not explosive. In order to achieve this, we choose $\lambda(0)$ such that the explosive root $\mu_{2}$ does not determine the evolution of the system for $t>T$. This ensures that we are on the stable path. Otherwise, the path for $k$ will be explosive. This restriction on $\lambda(0)$ is as follows:

$$
\frac{\Gamma_{k, \lambda}^{P C} \lambda(0)+\left(\mu_{2}-\Gamma_{\lambda, \lambda}^{P C}\right) k(0)}{\left(\mu_{2}-\mu_{1}\right)}=-\frac{\Gamma_{k, \lambda}^{P C} b_{\lambda, a}^{P C}+\left(\mu_{2}-\Gamma_{\lambda, \lambda}^{P C}\right) b_{k, a}^{P C}}{\mu_{2}\left(\mu_{2}-\mu_{1}\right)} e^{-\mu_{2} T}
$$

Studying the above equation in conjunction with the time paths for $k$ and $\lambda$, it can be seen that the discontinuity of $a(t)$ does not cause a discontinuity in the time path of $\lambda$ or $k$. Instead, the discontinuity of the exogenous process shows up in the time paths as a non-differentiability (kink) at time $T$.

Using the time paths of $k$ and $\lambda$ above, along with the restrictions for a stable solution, we have the following lemmas.

Lemma 4. Suppose the economy experiences a positive technology news shock and $v>v^{*}$. $\dot{\lambda} \geq 0$ and $\dot{k} \geq 0 \forall t<T$ if and only if $\lambda(0)>0$.

Lemma 5. Suppose the economy experiences a positive technology news shock and $v>v^{*}$. $\lambda(0)>0$ if and only if $\sigma<\sigma^{*}$, where $\sigma^{*}$ solves

$$
\mu_{2}^{P C}=(\rho+(1-\alpha) \delta) v /\left(\gamma_{I}+\sigma\right)
$$

and $\mu_{2}^{P C}=\mu_{2}^{P C}(\sigma)$ is the positive eigenvalue of $\Gamma^{P C}$.

The condition that $\sigma<\sigma^{*}$ implies that in order to generate procyclical technology news shocks the model requires a relatively low curvature parameter $\sigma$.

Lemmas 1 through 5 lead to the following theorem.

Theorem 1. The production complementarity model exhibits procyclical technology news shock if and only if $v>v^{*}$ and $\sigma<\sigma^{*}$. 
To better understand this theorem, Figure 5 plots the phase diagram for four cases. For now, we focus on the first three: (a) no production complementarity, (b) complementarity with a high curvature parameter, (c) complementarity with a low curvature parameter. Only case (c) results in procyclical news shocks. Initially before the news, as seen in in Figure $5(\mathrm{a})$, the capital-multiplier pair $\left(k_{s s}, \lambda_{s s}\right)$ lies on the initial manifold $\mathcal{M}$ and is at the initial steady state. Upon the time zero news arrival, the multiplier falls below $\lambda_{s s}$ because there is an immediate consumption boom. Because capital is a stock variable, $k_{0}=k_{s s}$. After time zero and before $T$ (which is the instant of the technology arrival), the capital stock falls indicating that investment is below the steady-state. The consumption boom comes at the expense of investment. This is one indicator of the comovement problem in the standard model: investment declines upon the arrival of good news.

Note that between time zero and time $T$, the capital-multiplier pair flow in the opposite direction of the stable manifold. This is due to influence of the explosive root $\left(\mu_{2}\right)$ before the technology change occurs. At time $T$, the model is on the new stable manifold $\mathcal{M}^{\prime}$ and the system then converges monotonically to the new steady-state. The $\mathcal{M}$ and $\mathcal{M}^{\prime}$ manifolds are parallel to each other because the technology shock does not change the coefficients multiplying the endogenous variables.

Figure 5(b) contains the phase diagram with production complementarity, but a high curvature parameter. As in the case without complementarity, investment initially falls in response to the news. Investment eventually increases once the technology change actually occurs. At this point, the system is on the manifold and capital converges monotonically to the new higher steady state. The desire for smooth consumption, due to the high curvature parameter, is evident in the path of the multiplier. It jumps downward on impact and then moves monotonically to the new steady state.

Figure 5(c) contains the phase diagram for the case of greatest interest. It represents the case when both conditions of Theorem 1 are satisfied: strong production complementarity and a low curvature parameter. Note that the marginal utility of investment jumps up rather than down in response to the good news.

Both $\sigma$ and $\delta$ play important roles in affecting $\lambda$ on impact, through equation (10). ${ }^{16}$ As a starting point, note first that according to the consumption-hours Euler equation (9), $n$ increases if and only if $i-c$ increases.

\footnotetext{
${ }^{16}$ The $L_{2}$ line is exactly equation (10) once the labor term is substituted out using (9).
} 
Next, rewriting equation (10), we have:

$$
\underbrace{\lambda}_{M U_{I}}=\underbrace{(v-1)(i-c)}_{\text {price }_{I}}+\underbrace{\left(-\sigma c-\frac{(1-\sigma)(1-\alpha)}{\left(1-s_{I}\right)} n\right)}_{M U_{C}}
$$

First, a good news shock that causes hours to rise is accompanied by an increase in the price of investment (in units of consumption) when $v>1$. This works to raise the marginal utility of investment. Next, consumption also rises if the news shock is procyclical. The increases in $c$ causes the marginal utility of consumption to fall, which offsets the price effect on $\lambda$. This effect is dampened when $\sigma$ is close to zero. This is a straightforward channel operating in the standard neoclassical model. ${ }^{17}$ Intuitively, when $\sigma$ is close to zero the timing of investment is governed by production efficiency concerns and not a desire to smooth consumption.

Finally, $n$ appears in the $M U_{C}$ term because consumption and hours are non-separable in the utility function. $\sigma$ plays a different role in the term pre-multiplying $n$. Here, it effects the degree of complementarity between $n$ and $c$ in preferences. If $\sigma=1$, preferences are separable and the $n$ term drops out. If $\sigma<1$, then leisure and consumption are complements, which puts downward pressure on $\lambda$. It must be the case that effect of $\sigma$ on $c$ is dominates its effect on $n$.

Thus, a low $\sigma$ (through a dampened consumption effect) and the production complementarity lead to an overall increase the shadow value of investment. This makes the return to forgoing leisure in order to produce the investment good high, which serves the requirement of Condition II.

Although investment jumps up at time zero, the new steady state must involve $k_{s s}^{\prime}>k_{s s}$ and $\lambda_{s s}^{\prime}<\lambda_{s s}$. This occurs in case (c) because the new manifold eventually crosses into the fourth quadrant of the phase space.

It is important to note that the conditions of Theorem 1 are independent of the value of $T$. As a result, the model preserves the ability to generate procyclical comovements in response to traditional time zero unanticipated shocks and news shocks for any time into the future. Many models that can generate procyclical comovements in response to news shocks are sensitive to the value $T$.

In addition to providing both the necessary and sufficient conditions for solving the news shock puzzle, Theorem 1 provides insight into understanding how news shocks work. In particular, the analysis sheds light on other mechanisms that are capable of solving the news

\footnotetext{
${ }^{17}$ If consumption and hours were separable, then $\sigma$ is simply the inverse of the intertemporal elasticity of substitution of consumption.
} 
shock puzzle. We present our understanding via the following observation.

Main Observation ${ }^{18}$ A variant of the neoclassical model will exhibit procyclical technology news shocks if it has one or more features that ensures:

I. consumption does not crowd out investment, or vice versa, and

II. the benefit to forgoing leisure is sufficiently strong.

Next, we consider the marginal effects of changing each of the model's remaining parameter. First, calibrating to a higher value of capital share, $\alpha$, decreases the cost to forgoing leisure and thus now allows relatively higher values of $\sigma$ to generate procyclical news shocks.

Figure 6 plots the minimum curvature parameter needed for $\lambda(0)>0$ when we we parameterize $\nu=\infty$ in our production based model. ${ }^{19}$ As the capital share increases, the required utility curvature parameter falls. A higher capital share in production decreases the cost to forgoing leisure and thus allows a lower curvature parameter to generate $\lambda(0)>0$.

Similarly, as $\rho / \delta$ increases, the minimum required intertemporal elasticity of substitution falls. An increase in $\rho / \delta$ implies either an increase in $\rho$ or a decrease in $\delta$. An increase in $\rho$ weakens the consumption smoothing motive and strengthens the returns to forgoing leisure. An increase in $\rho / \delta$ allows the model to exhibit procyclical news shocks with relatively low values of $\sigma$.

The second condition for a model to exhibit procyclical news shocks simplifies dramatically when the depreciation rate is near zero.

Lemma 6. For $\delta$ sufficiently close to zero, the model exhibits procyclical news shocks if $v>v^{*}$ and $\sigma<1$.

What is the role of the depreciation rate? Intuitively, if the capital depreciation rate is high, then the benefit to forgoing leisure is low. This is because, upon the arrival of the news, forgoing leisure to increase investment is only optimal if investment undertaken right away

\footnotetext{
${ }^{18}$ Lemma 2 and 4 tell us that $\lambda(0)>0$ is both a necessary and sufficient condition to generate procyclical technology news shocks in a standard neoclassical model with production complementarities. However, most economic frictions/features that result in $\lambda(0)>0$ have more than just that marginal effect on the model. Such frictions often also change the static and dynamic relationships in the standard neoclassical model with production complementarities. As a result of which we state this result as an observation rather than a theorem.

${ }^{19} \nu=\infty$ in the Leontief case. In this case $\lambda(0)>0$ if and only if $\sigma<\sigma_{c} . \sigma_{c}=-\xi_{A}+\sqrt{\xi_{A}^{2}+\xi_{B}}$, where $\xi_{A}=\frac{(2-3 \alpha)(1-\alpha)+\left(3(1-\alpha)+\alpha^{2}\right) \frac{\rho}{\delta}+\left(\frac{\rho}{\delta}\right)^{2}}{2(1-\alpha) \alpha}$ and $\xi_{B}=\frac{\left(1+\frac{\rho}{\delta}\right)\left((1-\alpha)^{2}+\frac{\rho}{\delta}\right)}{(1-\alpha) \alpha}$.
} 
has a positive effect on the capital stock once the technology actually increases; however, if the capital depreciation rate is too high, then much of the investment made at the arrival of the news will have depreciated by the time technology actually increases. A low depreciation rate implies that the forgone leisure used to produce the investment good is not depreciated away by the time of the technology arrival.

The importance of $\sigma$ in Lemma 6 was explained previously in the discussion of Theorem 1. A low $\sigma$ lessens the negative effect of the consumption boom on $\lambda$, allowing the positive price effect to dominate and thus increase $\lambda$.

Next, we plot the impulse responses for a specific model parameterization. Our model calibration meets the two conditions: $v>v^{*}$ and $\sigma<\sigma^{*}$. First, $v=1.8$. Vall'es (1997) finds that $v=1.8$ best matches the estimated responses of investment to various shocks. Sims (1989) uses a similar $F(C, I)$ function and chooses $v=3$.

The value of $\sigma$ in our baseline calibration is 0.5 , which implies less curvature than the oft-used 1.0 (i.e. $\log$ utility). However, $\sigma=0.5$ is within the range of some empirical estimates (e.g. Beaudry \& Wincoop (1996), Vissing-Jorgensen \& Attanasio (2003) and Mulligan (2002)). In Section 4, we consider modifications of the production complementarity model that allow for greater curvature in the utility function. The remaining parameters are less crucial and entirely in line with existing research. All parameters are reported in Table 1 .

The impulse responses are given in Figure 7. At time zero, capital is at the initial steady state and agents receive news of an expected one-percent permanent increase in technology that will arrive at $T=4$. Examining panels (b), (c) and (d), we see that consumption, hours and investment all increase on impact. Moreover, as our phase diagram and theorem dictate, the shadow value of investment $\lambda$ increases upon the arrival of the news (see panel (f)).

The response of consumption, seen in panel (b), is positive but nearly zero. This may be viewed as a deficiency of the model, although we note that non-durable consumption, the closest analogue in actual data to consumption in our model, contributes very little to empirical business cycles. In Section 4, we examine how adding various features to the baseline production complementarity model can affect the impulse responses quantitatively.

Finally, the model exhibits a large increase in consumption, investment, and hours upon the actual technology increase. 


\section{Procyclical News Shocks via a Positive Labor Exter- nality}

This section modifies the preference side of the growth model as a way to sustain procyclical news shocks. Specifically, we replace the momentary utility function in equation (4) with:

$$
W(C, N, \bar{N})=(1-\sigma)^{-1}\left[C \exp \left(-N \bar{N}^{-\gamma_{N}}\right)\right]^{1-\sigma}
$$

where $0<\gamma_{N}<1$ and $\bar{N}$ is the average economy-wide labor input. ${ }^{20}$ Thus, there is an external effect of employment on utility. In particular, the marginal disutility of labor falls as average labor rises. From an individual's perspective, he would prefer to work additional hours when others are working. In turn, the labor externality will act, from the household perspective, similarly to a preference shock that shifts out labor supply. This mechanism will ensure a low relative cost of forgoing leisure

Also, we add investment adjustment costs by replacing (3) with

$$
\dot{K}=I-\delta K-\frac{\psi_{I}}{2}\left(1-\frac{I}{\delta K}\right)^{2} I
$$

This is because no stable dynamic solution exists at low values of $\sigma$. With the employment externality, production complementarity is not necessary to support procyclical news shocks; as such, we set $\nu=1$.

The log-linearized equations that characterize a solution to the constrained planner's problem are now:

$$
\begin{gathered}
n=\frac{s_{I}}{1-\gamma_{N}}(i-c) \\
\underbrace{\lambda}_{M U_{I}}=\underbrace{\psi_{I}(i-k)}_{\text {price }_{I}}+\underbrace{(-\sigma c-z n)}_{M U_{C}} \\
\dot{\lambda}=-(\rho+\delta)\left[\left(1-s_{I}\right)(c-i)+i-k\right]+\rho \psi_{I}(i-k)
\end{gathered}
$$

as well as the resource constraints given by equations (12) and (13).

\footnotetext{
${ }^{20}$ To characterize the resource allocation in the presence of the externality, we have the social planner take the time path of $\bar{N}$ as given when choosing the time paths of $(C, I, K, N)$. Thus, we are studying a constrained optimal plan. It is straightforward to show that this allocation is the same as what would obtain in a competitive equilibrium with the externality.
} 
The term $\gamma_{N}$ only appears in (22), the consumption-labor Euler equation. ${ }^{21}$ and the addition of investment adjustment costs only alters equations (23) and (24).

We can substitute (22) into (12) to get a new consumption-investment production frontier (new $L_{1}$ line):

$$
\left(1-\phi_{I}^{L E}\right) c+\phi_{I}^{L E} i=\alpha k+(1-\alpha) a
$$

Here, $\phi_{I}^{L E}=\left(1-\frac{1-\alpha}{1-\gamma_{\bar{N}}}\right) s_{I}$. In this model, $L_{1}$ is upward sloping if $\phi_{I}^{L E}<0$. This requirement, which simplifies to $\gamma_{N}>\alpha$, is necessary and sufficient for comovement (although not necessarily procyclical) in response to a news shock.

Lemma 7. Suppose the economy experiences a positive technology news shock. Consumption, investment and hours comove at time zero if and only if $\gamma_{N}>\gamma_{N}^{*}=\alpha$.

Lemma 7 is the labor externality counterpart to Lemma 1 for the production complementarity model.

The steps in characterizing the allocation under labor externalities are very similar to those (previously done) under production complementarities. Two of the five equations, as noted above, are identical across the two cases. The remaining three equations contain nearly the same endogenous variables as in the previous model. Differences between the two sets of preferences are limited to the coefficients multiplying the endogenous variables. As such, we can apply the previous technique to this model.

The optimal solution satisfies the following conditions:

$$
\begin{gathered}
x=\tau_{x, k}^{L E} k+\tau_{x, \lambda}^{L E} \lambda+\tau_{x, a}^{L E} a \text { for } x=c, i, n \\
{\left[\begin{array}{c}
\dot{k} \\
\dot{\lambda}
\end{array}\right]=\Gamma^{L E}\left[\begin{array}{c}
k \\
\lambda
\end{array}\right]+b^{L E} a}
\end{gathered}
$$

The explicit formulas for $\tau_{,, \cdot}^{L E}, b^{L E}$ and $\Gamma^{L E}$ are given in Appendix B.

As mentioned previously for a model with labor externalities there exists no stable dynamic solution for low values of $\sigma$. The addition of investment adjustment costs alleviates this problem. The next lemma holding fixed all other parameter values gives the minimum value of $\psi_{I}$, the investment adjustment cost parameter, under which a stable dynamic solution exists.

\footnotetext{
${ }^{21}$ In fact, if one were to replace $1 /\left(1-\gamma_{N}\right)$ with the value $\nu$ on the left-hand side, the equation would be identical to (9) from the production complementarity model.
} 
Lemma 8. Suppose the economy experiences a positive technology news shock. Also, assume that $\gamma_{N}>\gamma_{N}^{*}$. Then a stable solution to equation (26) exists if

$$
\psi_{I}>\psi_{I}^{+}=-\frac{\gamma_{I}^{L E}+\phi_{I}^{L E} \sigma}{1-\phi_{I}^{L E}}
$$

Lemma 9. Suppose the economy experiences a positive technology news shock. Also, assume that $\gamma_{N}>\gamma_{N}^{*}$ and $\psi_{I}>\psi_{I}^{+} . \lambda(0)>0$ if and only if $\psi_{I}>\psi_{I}^{*}$. where, $\psi_{I}^{*}$ solves equality

$$
\mu_{2}^{L E}=\frac{\Gamma_{\lambda, \lambda}^{L E} b_{k, a}^{L E}-\Gamma_{k, \lambda}^{L E} b_{\lambda, a}^{L E}}{b_{k, a}^{L E}}
$$

and $\mu_{2}^{L E}=\mu_{2}^{L E}\left(\psi_{I}\right)$ is the positive eigenvalue of $\Gamma^{L E}$.

Theorem 2. The labor externality model exhibits procyclical technology news shock if: $\gamma_{N}>$ $\gamma_{N}^{*}$ and $\psi_{I}>\operatorname{Max}\left\{\psi_{I}^{+}, \psi_{I}^{*}\right\}$.

Here is the intuition and relation to our main observation. As labor increases, the marginal product of labor falls, which reduces the incentive to work. Without an external effect, the marginal disutility of labor is increasing in labor. Thus, these two effects work in the same direction. On the other hand, with the external effect, in a symmetric equilibrium, the effective marginal disutility of labor is falling in labor. If the labor externality, measured by $\gamma_{N}$, has a stronger positive labor supply effect than the negative diminishing returns to labor demand effect, measured by $1-\alpha$, then the employment increase will be sufficiently large to support both a consumption and investment increase. ${ }^{22}$ Thus, the labor externality mechanism achieves Condition I.

Next, equation (23) is critical for ensuring that the marginal utility of investment rises on impact. The intuition here is identical to that of the production complementarity model with one difference. In the current model, the price of investment (see the right-hand side of equation (23)) increases because of investment adjustment costs term, $\psi_{I}(i-k)$. In the production complementarity model, the price of investment (see the right-hand side of equation (20)) because of the production complementarity term, $v(i-c)$. The adjustment cost mechanism achieves Condition II.

\footnotetext{
${ }^{22}$ For parameter pairs $\left(\gamma_{N}, \alpha\right)$ that satisfy the comovement condition, note that there does not exist an interior solution to the first-best resource allocation. With $\gamma_{N}>0$, the social marginal disutility of labor is declining, rather than rising, as labor increases. With $\gamma_{N}>\alpha$, this decline occurs more rapidly than the increase in marginal cost associated with diminishing returns.
} 
Although the labor externality model has a different mechanism than the production complementarity model, the relevant diagrams for the two models are identical. Figure 5(c) is the correct phase diagram for the new model; Figures 3 and 4 illustrate the static relationships for the new model.

Next, we plot the impulse responses for a specific model parameterization. Our model calibration meets the requirement $\gamma_{\bar{N}}>\alpha$ and the two conditions on $\psi_{I}$. We set $\gamma_{N}=$ $0.332, \psi_{I}=20$ and $\sigma=1$. All parameters values are reported in Table 1 .

The values of $\sigma$ and $\psi_{I}$ are in line with thos of existing research. On the other hand, $\gamma_{N}$ is chosen somewhat arbitrarily because existing research provides no guidance for choosing its value. This parameter choice is motivated by our desire to demonstrate one mechanism capable of supporting procyclical news shocks.

The impulse responses are given in Figure 8. Consumption, hours, investment as well the shadow value of investment all increase upon the arrival of the news. Quantitatively, we view the results as disappointing. Each of the three main variables is nearly acyclical in response to the positive news.

\section{Quantitative Issues and Calibration Issues}

Section 2 established that adding production complementarity to the neoclassical growth model was sufficient to support procyclical news shocks. The mechanism, by itself, has two potential drawbacks: the resulting consumption boom is miniscule and it requires low curvature in the utility function. Both drawbacks are also present in the preference-externality model. This section restricts attention to the production complementarity model and shows how either changing preferences or adding investment adjustment costs can mitigate these difficulties.

\section{Greenwood-Hercowitz-Huffman Preferences}

Suppose we replace the King, Plosser and Rebello preferences (1988, hereafter KPR), used earlier in this paper, with those of Greenwood, Hercowitz and Huffman (1988, hereafter $\mathrm{GHH}$ ). The instantaneous utility function becomes

$$
V(C, N)=\left(C-\xi \frac{N^{1+\psi}}{1+\psi}\right)^{1-\omega} /(1-\omega)
$$

where $\omega, \xi, \psi>0$. The set-up is otherwise identical to the production-complementarity model. 
The critical feature of these preferences is that the marginal disutility of labor is falling in consumption. Recall that, in absence of a low $\sigma$ under the KPR preferences, it was optimal for the planner to delay the hours boom until the new technology arrives. Under GHH preferences, the marginal disutility of work is falling in consumption; as such, there is an preference-driven incentive to work more during a consumption boom.

The log-linearized conditions for an optimum consists of five equations, three of which are identical to the baseline production-complementarity model. ${ }^{23}$ The first equation (that does change) is the intratemporal consumption-hours Euler condition. Under GHH preferences, it is:

$$
n=v s_{I}(i-c)+c
$$

For comparison, we restate the corresponding equation for the baseline preferences:

$$
n=v s_{I}(i-c)
$$

The production-complementarity effect, reflected by the $v s_{I}(i-c)$ term, is present in both equations. GHH preferences, additionally, imply that the planner works more hours when consumption is high. This augments the model's ability to achieve Condition I.

The second equation (that does change) is the consumption-investment Euler condition. Under GHH preferences, it is 24

$$
\lambda=(v-1)(i-c)+\underbrace{\frac{1}{1-s_{n}}\left[-\omega c+\omega s_{n} n\right]}_{M U_{C}}
$$

For the baseline preferences, we have

$$
\lambda=(v-1)(i-c)+\underbrace{\left[-\sigma c-(1-\sigma)\left(\frac{1-\alpha}{1-s_{I}}\right) n\right]}_{M U_{C}}
$$

Recall that in order for comovement to be procyclical with respect to positive news, $\lambda$ must increase upon arrival of the news. This is an implication of our Condition II. Under the baseline preferences, we showed that this was qualitatively possible if $v$ was sufficiently large and $\sigma$ was sufficiently close to zero; however, quantitatively the consumption boom was nearly very small. Examining the KPR preferences, one sees that $\lambda$ is decreasing in

\footnotetext{
${ }^{23}$ The unchanged equations are (11), (12), and (13).

${ }^{24}$ Here $s_{n}=\frac{(1-\alpha)}{1-s_{I}}$
} 
consumption. Roughly speaking, the planner attempts to allocate output to consumption and investment to equalize their marginal benefit. If consumption rises too much, then the marginal utility of consumption, $M U_{c}$, will fall too much. In turn, the marginal utility of investment would also have to fall.

GHH preferences help support an increase in $\lambda$ in response to positive news. This is because, while $M U_{c}$ is falling in consumption, it is rising in hours worked for any $\omega$. Since labor and consumption comove, this leads to an offsetting effect on $M U_{c}$.

The steps in characterizing the optimal allocation under GHH preferences are very similar to those described previously. Three of the five equations, as noted above, are identical across the two cases. The remaining two equations, (27) and (28), for the GHH-preferences case, contain the same endogenous variables as in the KPR case. Differences between the two preference assumptions are limited to the coefficients multiplying the endogenous variables. As such, we can apply the previous technique.

The optimal solution satisfies the following conditions:

$$
\begin{gathered}
x=\tau_{x, k}^{G H H} k+\tau_{x, \lambda}^{G H H} \lambda+\tau_{x, a}^{G H H} a \text { for } x=c, i, n \\
{\left[\begin{array}{c}
\dot{k} \\
\dot{\lambda}
\end{array}\right]=\Gamma^{G H H}\left[\begin{array}{c}
k \\
\lambda
\end{array}\right]+b^{G H H} a}
\end{gathered}
$$

The explicit formulas for $\tau_{.,}^{G}$ and $\Gamma^{G}$ are given in Appendix B. Appendix B also contains the conditions on the underlying parameters $(\alpha, \omega, \rho, \delta, \nu, \xi)$ required for the model to exhibit procyclical news shocks.

Next, we examine the quantitative implications of applying GHH preferences. The three new model parameters are set at $\omega=0.5, \psi=0.01$, and the scale parameter $\xi=6.96$ to match a steady state value of labor $N=0.3$. We calibrate the remaining parameters at the values used in Section 2.

\section{Investment Adjustment Costs}

Condition II to generate procyclical news shocks requires a model feature that will ensure the benefit to forgoing leisure sufficiently outweighs the cost. Parameterizing our model with a high intertemporal elasticity of substitution led to us satisfy this condition in our basic production-based model. Further analysis of the marginal effect of other parameters in that model showed that increasing the marginal returns to investment, either by increasing the capital share in production or lowering the depreciation rate, also led to an increase in the 
benefit to forgoing leisure, albeit not large enough ${ }^{25}$. The natural extension thus would be to include a feature that generates a very high returns to investment such as investment adjustment costs.

We make two modifications to the baseline production-complementarity model. First, we introduce investment adjustment costs by replacing (3) with (21). Second, we set $\sigma=1$ to give us standard log utility in consumption.

This addition alter equations (10) and (11) in our log-linearized system by adding a new term:

$$
\begin{gathered}
\lambda=\underbrace{(v-1)(i-c)+\psi_{I}(i-k)}_{\text {price }_{I}}+\left(-\sigma c-(1-\sigma)\left(\frac{1-\alpha}{1-s_{I}}\right) n\right) \\
\dot{\lambda}=-(\rho+\delta)\left[v\left(1-s_{I}\right)(c-i)+i-k\right]+\rho \psi_{I}(i-k)
\end{gathered}
$$

The remaining three equations are unchanged.

Equation (29) gives us the optimal consumption-investment decision. It is identical to the baseline production-complementarity model except there is an additional component to the price of investment, $\psi_{I}(i-k)$. This component is due to the investment adjustment costs, is increasing in $i$ and works to increase $\lambda$. The phase diagrams in Section 2 showed why an increase in $\lambda$ upon arrival of the news is required in order that a model support procyclical comovement. Adjustment costs help ensure that increase in $\lambda$, and, therefore, Condition II.

Equation (30) is the intertemporal consumption Euler equation. The sole difference in this equation from the baseline production-complementarity model is that the rate of change in the marginal utility of investment reflects the investment adjustment cost.

Next, consider the impact of adjustment costs on Condition I. Even though the addition of investment adjustment costs alters equation (10), and thus the static system, it can be shown that the Lemmas 1 through 4 still hold. The $L_{1}$ line, equation (15), remains the same, and while the magnitude of the slope of $L_{2}$ changes qualitatively, there is no qualitative change. Figures $3(\mathrm{~b})$ and 4 still reflect (qualitatively) the static and required dynamic relationship in the adjustment cost model.

With investment adjustment costs the dynamic analysis is slightly altered. Lemma 5 , and thus Theorem 1, now place a different parameter restriction required to generate a positive $\lambda(0)$. Most importantly the restriction on the critical value of $\sigma$ is relaxed. High investment

\footnotetext{
${ }^{25}$ We still need higher than normal values of the intertemporal elasticity of substitution
} 
adjustment costs lead to an increase in the returns to investment, which in turn leads to more capital, which further in turn leads to an increase in the benefit of supplying labor and forgoing leisure. Finally, the dynamic analysis in the $(k, \lambda)$ space is qualitatively still given by Figure 5 .

\section{Forward-Compatible Investment}

One observation, thus far, has been that in order to generate procyclical news shocks we require a feature or features that increases $\lambda(0)$, which is an alternative expression of Condition II. In this section, we describe how this can be achieved by introducing forward-compatible investment. Physical investment is forward-compatible if additions to the capital made between the arrival of the news and the actual technology increase are particularly well-suited to the future technology. For example, if an IT firm is laying down fiber optic cables and it knows a new, better standard will be in effect in a year then it may be able to ensure that the fiber optic cables currently being installed can take advantage of the new standard. This will mean that at least part of the investment done in the preparatory phase $(t \in[0, T))$ will be able to have an advantage of the new technology improvement once time $T$ arrives.

\section{The Model}

Suppose that at time zero, news arrives of a future investment-specific technology shock. ${ }^{26}$ The technology increase will arrive at $T>0$ and will be permanent:

$$
Q(t)=\left\{\begin{array}{cc}
\bar{Q} & \text { for } t \in[0, T) \\
\tilde{Q}=1.01 \times \bar{Q} & t \geq T
\end{array}\right.
$$

We shall refer to time between zero and $T$ as the preparatory phase.

This shock appears in the capital law of motion:

$$
\dot{K}(t)=Q(t) I(t)-\delta K(t)+\left(K(t)-e^{-\delta T} \bar{K}\right) P(\tilde{Q}, t, T, \epsilon)
$$

where $\bar{K}$ is the initial capital stock, which we assume is at the steady-state consistent with $\bar{Q}$.

The right-hand side of (32) decomposes the time derivative of capital into three terms. The first term is the contribution of investment multiplied by the current efficiency of invest-

\footnotetext{
${ }^{26}$ Investment-specific technology shocks by themselves cannot generate news-driven procyclical business cycles, established in Beaudry and Portier (2007).
} 
ment. The second is the capital lost due to depreciation. The last term embodies the model's key assumption. It is the multiple of two functions. The first function is $K(t)-e^{-\delta T} \bar{K}$, representing the investment accumulated during the preparatory phase that has not depreciated. For convenience, define $\mathcal{K}(t)=K(t)-e^{-\delta T} \bar{K}$. The second function, $P(Q, t, T, \varepsilon)$, is a positive technology shock. It will be constructed so that it permanently increases the productivity of investment made during the preparatory phase. Thus, investment made after the arrival of news will be forward-compatible with the yet-to-arrive technology.

This second function will be non-zero only in a small neighborhood of $T$. Thus, this positive shock will not come online until time $T$. The contribution of the third term in (32) will only apply to $\mathcal{K}(T)$. We refer to $\mathcal{K}(T)$ as partially-adapted capital. We plot the log deviations of the functions $Q$ and $P$ in Figure 10.

$P$ depends on $t$ because the forward-compatible effect is only operative for a certain interval of time. The term $\varepsilon$ is a small positive number. It will define the neighborhood (of the time interval) in which the "forward-compatibility" shock occurs. Mathematically, $P(Q, t, T, \varepsilon) \neq 0$ only if $t \in[T, T+\varepsilon]$. A technical detail neccesitates $\varepsilon{ }^{27}$ Later, we will drive $\varepsilon$ to zero at the appropriate rate. We choose a particular form for $P$ to aid calibration:

$$
P(\tilde{Q}, t, T, \varepsilon)=\left\{\begin{array}{cc}
0 & \text { for } t \in[0, T) \cup(T+\epsilon, \infty) \\
\frac{\tau \tilde{Q}}{\left(1-e^{-\epsilon}\right) \bar{Q}} & t \in[T, T+\epsilon]
\end{array}\right.
$$

For notational simplicity, we will sometimes use $P_{\varepsilon}(t)$ and suppress the function's dependence on $\tilde{Q}$ and $T$.

A positive investment-specific technology shock is isomorphic to a positive neutral technology shock combined with a capital depreciation shock. Forward compatability, in the face of an investment-specific news shock, mitigates where the "capital depreciation" shock component. That is, capital put in place following the news will not depreicate to the same degree as the already in-place capital. Thus, forward compatability, by itself, boosts the relative benefit to forgoing leisure to produce investment upon the news arrival.

Production of consumption occurs according to equations (1) and (2); that is, there is production complementarity between consumption and investment. ${ }^{28}$ The utility function is (4), which is taken from the baseline model of Section 2.

\footnotetext{
${ }^{27}$ If $P$ were positive and finite only at one instant, then $P$ will have zero effect on the capital. Instead, as we let $\epsilon$ approach zero, the $P$ will become infinite at the instant $T$ and cause $K$ to jump upward. This technical detail is not needed in a discrete time model.

${ }^{28}$ We assume that $A_{t}=1$, since technology improvements are only investment specific in this model.
} 


\section{The Planning Problem and Its Solution}

The social planner chooses $C, I, K$ and $N$ to maximize $U$ subject to (1), (2) and (32), taking as given the initial condition $K(0)$ and time path of technology. The current value Hamiltonian for the problem is:

$$
H=(1-\sigma)^{-1} C^{1-\sigma} \exp [-(1-\sigma) N]+\Lambda\left(Q I-\delta K+P_{\varepsilon} \mathcal{K}\right)+\Phi\left(K^{\alpha} N^{1-\alpha}-F(C, I)\right)
$$

The log-linearized system that solves this Hamiltonian is given by five equations. ${ }^{29}$ Two equations, (9) and (12), are identical to those from the baseline production complementarity model. These are the consumption-hours Euler equation and the production function. The three new equations are:

$$
\begin{gathered}
\lambda=(v-1)(i-c)+(-\sigma c-z \nu)-q \\
\dot{\lambda}=-(\rho+\delta)\left[v\left(1-s_{I}\right) c+q+i-k\right]-p_{\varepsilon} \\
\dot{k}=\delta(q+i-k)+\left(1-e^{-\delta T}\right) p_{\varepsilon}
\end{gathered}
$$

Equation (33) ensures an efficient consumption-investment split. It is identical to the corresponding equation from the baseline production complementarity except for the final term on the right-hand side, $-q$. This is a relative price effect because technology is investmentspecific. Before time $T$, however, $q(t)=0$ because the technology improvement has not yet arrived.

Equation (34) is the intertemporal consumption Euler equation. It differs from the neoclassical model in two ways. First, $\lambda$ is not simply the derivative of the marginal utility of consumption. There is an additional relative price effect because of the convex production frontier. Second, $\lambda$ jumps down at $T$ as a result of $p_{\varepsilon}$, that is in the limit as $\varepsilon \rightarrow 0$.

Equation (35) is the law of motion for capital. On the right-hand side, the first term is standard and the second reflects an increase in capital at time $T$. This occurs when the forward-compatible capital built during the preparatory phase becomes utilized with the new technology. Recall that $p_{\varepsilon}$ is positive, and otherwise zero, only in a neighborhood of $T$. Below, we take the limit as $\varepsilon \rightarrow 0$. Then, $p_{\varepsilon}$ becomes "infinite at an instant," causing an upward jump in the capital stock. The optimal solution also satisfies a standard transversality condition and an initial condition on capital.

\footnotetext{
${ }^{29}$ The non-linear first-order conditions are presented in the appendix.
} 
Recall that, in order to support procyclical news shocks, a model must have mechanism(s) that ensure: (I) consumption does not crowd out investment, and (II) a sufficiently large return to forgoing leisure.

Production complementarity is sufficient to ensure Condition I due to the derivations in the benchmark production complementary model. Following those derivations (detailed in the appendix), we have expressions for the jump variables (consumption, investment and hours) as functions of the state variables.

$$
x=\tau_{x, k}^{F C} k+\tau_{x, \lambda}^{F C} \lambda+\tau_{x, q}^{F C} q \text { for } x=c, i, n
$$

Substituting (36) into the $\dot{k}$ and $\dot{\lambda}$ equations, (34) and (35), we have:

$$
\left[\begin{array}{l}
\dot{\lambda}(t) \\
\dot{k}(t)
\end{array}\right]=\Gamma^{F C}\left[\begin{array}{l}
\lambda(t) \\
k(t)
\end{array}\right]+b_{q}^{F C} q(t)+b_{p}^{F C} p(\tilde{q}, t, T, \varepsilon)
$$

The explicit formulas for $\tau_{.,}^{F C}, \Gamma^{F C}, b_{q}^{F C}$ and $b_{p}^{F C}$ are given in the appendix.

Next, we provide a theorem concerning procyclical news shocks in the forward-compatibility model.

Theorem 3. The forward-compatible investment model exhibits procyclical technology news shocks if and only if $v(1-\alpha)>1$ and

$$
\tau>\frac{\Gamma_{k, \lambda}^{F C} b_{\lambda, q}^{F C}+\left(\mu_{2}^{F C}-\Gamma_{\lambda, \lambda}^{F C}\right) b_{k, q}^{F C}}{\Gamma_{k, \lambda}^{F C} \mu_{2}^{F C} b_{\lambda, p_{\epsilon}}^{F C}+\left(\mu_{2}^{F C}-\Gamma_{\lambda, \lambda}^{F C}\right) \mu_{2}^{F C} b_{k, p_{\epsilon}}^{F C}}
$$

where $\mu_{2}^{F C}$ is the positive eigenvalue of $\Gamma^{F C}$.

Let us understand this result: Theorem 3 requires that there is both sufficient complementarity in production (Condition I) and the new capital accumulated between time 0 and $T$ must be sufficiently forward compatible (Condition II). Solving the differential equation (37), the time-paths of $(k, \lambda)$ are: ${ }^{30}$

\footnotetext{
${ }^{30}$ The online Appendix B contains the derivation of (39) and (40)
} 


$$
k(t)=\left\{\begin{array}{cc}
\frac{\Gamma_{k, \lambda} \lambda(0)}{\mu_{1}-\mu_{2}} e^{\mu_{1} t}+\frac{\Gamma_{k, \lambda} \lambda(0)}{\mu_{2}-\mu_{1}} e^{\mu_{2} t} & \text { for } t \in[0, T) \\
\frac{\Gamma_{k, \lambda} \lambda(0)}{\mu_{1-} \mu_{2}} e^{\mu_{1} t}+\frac{\Gamma_{k, \lambda} b_{\lambda, q}-\Gamma_{\lambda, \lambda} b_{k, q}}{\mu_{1} \mu_{2}}+\frac{\Gamma_{k, \lambda}\left(b_{\lambda, q}+\tau \mu_{1} b_{\lambda, p_{\epsilon}}\right)+\left(\mu_{1}-\Gamma_{\lambda, \lambda}\right)\left(b_{k, q}+\tau \mu_{1} b_{k, p_{\epsilon}}\right)}{\mu_{1}\left(\mu_{1}-\mu_{2}\right)} e^{\mu_{1}(t-T)} & t \geq T
\end{array}\right.
$$

$$
\lambda(t)=\left\{\begin{array}{c}
\frac{\left(\mu_{1}-\Gamma_{k, k}\right) \lambda(0)}{\mu_{1}-\mu_{2}} e^{\mu_{1} t}+\frac{\left(\mu_{2}-\Gamma_{k, k}\right) \lambda(0)}{\mu_{2}-\mu_{1}} e^{\mu_{2} t} \\
\frac{\left(\mu_{1}-\Gamma_{k, k}\right) \lambda(0)}{\mu_{1}-\mu_{2}} e^{\mu_{1} t}+\frac{\Gamma_{\lambda, k} b_{k, q}-\Gamma_{k, k} b_{\lambda, q}}{\mu_{1} \mu_{2}}+\frac{\Gamma_{\lambda, k}\left(b_{k, q}+\tau \mu_{1} b_{k, p_{\epsilon}}\right)+\left(\mu_{1}-\Gamma_{k, k}\right)\left(b_{\lambda, q}+\tau \mu_{1} b_{\lambda, p_{\epsilon}}\right)}{\mu_{1}\left(\mu_{1}-\mu_{2}\right)} e^{\mu_{1}(t-T)}
\end{array}\right.
$$

where $\mu_{1}$ and $\mu_{2}$ are the eigenvalues of the $\Gamma^{F C}$ matrix. ${ }^{31}$

The above solution has one undetermined variable $\lambda(0)$. We seek a path for $(\lambda, k)$ that is not explosive. We choose $\lambda(0)$ such that the explosive root $\mu_{2}$ does not determine the evolution of the system for $t>T$; otherwise, the path would be explosive. This restriction on $\lambda(0)$ is:

$$
\frac{\Gamma_{k, \lambda} \lambda(0)+\left(\mu_{2}-\Gamma_{\lambda, \lambda}\right) k(0)}{\left(\mu_{2}-\mu_{1}\right)}=-\frac{\Gamma_{k, \lambda}\left(b_{\lambda, q}+\tau \mu_{2} b_{\lambda, p_{\epsilon}}\right)+\left(\mu_{2}-\Gamma_{\lambda, \lambda}\right)\left(b_{k, q}+\tau \mu_{2} b_{k, p_{\epsilon}}\right)}{\mu_{2}\left(\mu_{2}-\mu_{1}\right)} e^{-\mu_{2} T}
$$

Panel (d) of Figure 5 contains the phase diagram for the forward-compatibility model when the conditions of Theorem 3 are satisfied. Before the news shock, the economy is at its initial steady state $\left(k_{s s}, \lambda_{s s}\right)$. The initial capital stock is lower and the shadow value of investment is larger than their long-run, post-shock counterparts $\left(k_{s s}^{\prime}, \lambda_{s s}^{\prime}\right) . \mathcal{M}$ is the pre-shock stable manifold and $\mathcal{M}^{\prime}$ is the corresponding manifold after time $T$.

At the instant of the news arrival, the shadow value of investment increases. This occurs because new investment will be more productive relative to previous investment, albeit not until time $T$. Capital does not jump instantaneously; however $\dot{k}(0)>0$. This shows that investment increases in response to the shock - the first of three requirements for procyclical news shocks. The reader should return to Figure 3(b) to see graphically that the second requirement-that consumption rises-is satisfied. That figure shows that the increase in $\lambda$ results in higher consumption. ${ }^{32}$ The third requirement - that hours increase- holds because, otherwise, both consumption and investment could not increase.

\footnotetext{
${ }^{31}$ In the above expressions and the expression below, we suppress the superscript $F C$ from several variables to avoid notational clutter.

${ }^{32}$ The intuition for this effect is discussed early in the paper.
} 
At instant $T$, the actual increase in investment specific technology rises. All future investment produces an additional one percent of capital. Moreover, because of forward compatibility, investment made during the preparatory phase becomes $\tau$ percent more productive. This latter effect causes the capital to jump up at $T$. This latter effect also causes $\lambda$ to jump down from $T^{-}$to $T^{+}$. When the capital stock jumps up, the shadow value of investment, i.e. adding to that capital stock, declines in a discrete fashion. During the preparatory phase, the explosive root is operative, causing $\lambda$ to rise.

Next, at after time $T^{+}, k$ and $\lambda$ lie on the new stable manifold $\mathcal{M}^{\prime}$. After the news arrives, the system is saddle path stable. If $(k(t), \lambda(t))$ did not lie on $\mathcal{M}^{\prime}$ for $t>T^{+}$, then the pair would diverge. Intuitively, going from $T^{+}$onward, $k$ and $\lambda$ must be on the new manifold because technology has reached its new permanently higher level. ${ }^{33}$

To close this section, we discuss the main restriction on the model parameters:

$$
\tau>\frac{\Gamma_{k, \lambda} b_{\lambda, q}+\left(\mu_{2}-\Gamma_{\lambda, \lambda}\right) b_{k, q}}{\Gamma_{k, \lambda} \mu_{2} b_{\lambda, p_{\epsilon}}+\left(\mu_{2}-\Gamma_{\lambda, \lambda}\right) \mu_{2} b_{k, p_{\epsilon}}}
$$

In our above discussion, we have outlined that the dynamics crucially depend on the marginal returns on investment relative to consumption. In particular, we need a sufficiently large return to induce the planner to increase investment. Both the curvature in the utility function and the degree of complementarity affect the size of the marginal return on investment. Low curvature lessens the consumption-smoothing motive and, thus, reduces the cost of allocating resources towards investment and away from consumption. In the extreme case when $\sigma=0$, the planner no longer cares about consumption smoothing and his sole aim is to have capital at its new steady level at time $T$. This causes the return on investment to increase. Ideally, the planner would like to jump to the new capital level by only sacrificing consumption in a small neighborhood before $T$, however, production complementarity would cause the price of consumption at that instant to increase dramatically. This results in the planner smoothing the investment increase between time zero and $t=T$.

\section{Quantitative Analysis of the Forward-Compatibility Model}

\footnotetext{
${ }^{33}$ The partial forward-compatible assumption in this model is similar to the time-to-build assumption where $\dot{K}(t)=Q(t) I(t-\xi)-\delta K(t)$ (here $\xi$ gives a measure of how long it takes to build capital). There are two key differences though: (1) forward-compatible investment is available in the period after it is made, (2) only part of the current investment is able to take advantage of future technology in the forward compatible model. That said, it can be shown that in a model with production complementarity and time-to-build if $x i>T$ then under certain plausible calibrations the model is able to generate procyclical technology news shocks.
} 
The only new parameter is $\tau$, the degree of forward compatibility. This is clearly a difficult parameter to calibrate. We set $\tau=0.66$. The remaining parameters are set at values used earlier in the paper.

Figure 11 plots the responses of key variables to a positive news shock. As seen in panels (b)-(d), consumption, investment and hours all increase upon the arrival of the news. The three then increase smoothly until the beginning of quarter four. Then technology actually increases; each of the three jumps upward because the output cost of producing newly installed capital, $1 / Q$, falls.

Note that consumption increases by less than investment on impact. The magnitude of the consumption response is sensitive to parameter values. Specifically, the size of this response is increasing in the degree of forward compatibility, production complementarity and the curvature parameter.

Although we set $T=4$ (i.e. one year) in our baseline calibration, the model can exhibit procyclical news shows for much higher values of $T$. Holding all other parameters fixed at their benchmark values, calculations using Theorem 3, procyclical news shocks obtain for any $T<21.3$.

Next, the relative price of investment to consumption, seen in panel (e), rises on impact due to production complementarity along with a larger increase in investment than an increase in consumption in response to the news. Panel (f) shows that the shadow value of investment is procyclical.

The dynamics of news shocks of every model in this paper are contained in Figure 5. All models discussed in the paper, excluding the forward-compatible model, achieve $\lambda(0)>0$ by causing the stable manifold to shift upwards. With the forward compatibility, this is not necessary. We can maintain the stable manifold movements as in the standard neoclassical model and yet generate procyclical news shocks by instead adding in a friction that results in a discontinuity in the time path of the state and co-state variables. This allows a fair amount of flexibility in adding other non-related frictions and still generating procyclical news shocks.

Including a feature that adds a discontinuity to the state variable allows for a much richer set of dynamics and places fewer restrictions on the movements of the stable manifold vis-a-vis the inclusion of other economic frictions in the model.

With respect to existing research, we note that Flóden (2007) constructs a two-period model that is capable of resolving the comovement puzzle using variable capacity utilization and vintage capital. In his paper, news arrives in the initial period and technology arrives in 
the second period. The news increases the efficiency of investment made in the initial period toward producing capital in the second period, which is similar to our forward-compatibility.

We are additionally interested in the value of the capital stock, which can be interpreted as the value of the stock market in a typical decentralization of our social planner's problem. Beaudry and Portier (2006) suggest that the stock price is procyclical with respect to technology news shocks.

The value of the firm with forward compatible investment, in a decentralization of our model, is:

$$
V^{F C}(t)=\left(\frac{i(t)}{c(t)}\right)^{\nu-1} \frac{1}{q(t)} k(t)-I_{t<T}\left[e^{-\rho(T-t)} \frac{U_{C}(T)}{U_{C}(t)}\left(\frac{i(T)}{c(T)}\right)^{\nu-1} e^{-\delta T} \bar{K} \tau\right]
$$

We can break this equation into three distinct parts: $(i)\left(\frac{i}{c}\right)^{\nu-1}$ gives the price of investment due to the interplay between consumption-investment along the bowed-out production frontier that is generated by production complementarities in the model; $(i i) \frac{1}{q}$ gives a measure of the exogenous changes in the price of capital due to investment-specific shocks. Terms (i) and (ii) multiply $k$; (iii) the last term reflects the fact that with forward-compatible investment not all capital experiences a productivity jump when the news arrives.

The solid line in Figure 12 plots the impulse response of $V^{F C}(t)$. The value of the firm falls on impact. This is not surprising. In the standard neoclassical model, a contemporaneous positive investment-specific technology shock causes the value of the firm to fall. Recall the value of the firm is simply the value of its capital. Higher productivity in the capitalproducing sector depresses the price of capital. Thus, the value of the firm must fall on impact. This effect, represented by $(i i)$, operates under forward compatibility and with respect to news shocks as well. It is somewhat dampened by $i i$; the bowed-out production frontier and increase in the investment-consumption causes the price of capital to increase. Finally, term (iii) contributes to a fall in $V F C(t)$ upon the news arrival.

Neutral changes in technology, as opposed to investment-specific, do not necessarily reduce the price of capital relative to consumption. Consider the value of the firm in our model of Section 2. Recall that it contained neutral technology shocks and production complementarties. In that case, the value of the firm is $V^{P C}(t)=(i(t) / c(t))^{\nu-1} k(t)$. The dotted line in Figure 12 plots the impulse response for $V^{P C}(t) .{ }^{34}$ For this model, the value of the firm rises with the arrival of the shock. The presence of production complementarities increase the

\footnotetext{
${ }^{34}$ We use the benchmark calibration from Section 2 to compute this impulse response.
} 
price of investment relative to consumption when the planner moves to increase investment in response to a news shock. This suggests that models with neutral-technology news shocks are more likely to generate procyclical stock prices.

\section{Conclusion}

In this paper, we have made the case that an analytic approach to understanding news shocks in stochastic growth models helps to generate important insights. Up to a firstorder approximation, we have provided analytic solutions to five variants on the standard neoclassical model. In each case, we have provided conditions under which the model exhibits procyclical news shocks. For each model, we have provided a plausible calibration and then examined the quantitative features of the impulse response functions.

By examining several models, we have identified commonalities across models that resolve the news-shock comovement puzzle. Each model has a mechanism or mechanisms that ensure that consumption and investment do not crowd each other out and that the relative benefit of forgoing leisure outweighs its cost.

As a result of our analysis, we have developed a view on what type of mechanisms best suited for achieving the above conditions. First, we contend that it is more reasonable to attribute procyclical news shocks to production-based rather than preference-based components. For example, GHH preferences help significantly boost the size of a consumption boom in the production complementary model, using these preferences creates other problems, not related to news shock. These include an absence of balanced growth with respect to neutral technological change. On the other hand, the addition of production complementarity seems to be a plausible production-based mechanism to ensure that consumption and investment do not crowd each other out. 


\section{Bibliography}

Barsky, Robert B., and Eric Sims, "News Shocks and Business Cycles," Journal of Monetary Economics, 58:273-289, 2011.

Beaudry, Paul, Martial Dupaigne, and Franck Portier, "Modeling News-Driven International Business Cycles," Review of Economic Dynamics, 14:72:91, January 2011.

Beaudry, Paul and Bernd Lucke, "Letting Different Views about Business Cycles Compete," NBER Macroeconomics Annual 2009, 24:413-455, 2010.

Beaudry, Paul and Franck Portier, "An exploration into Pigou's theory of cycles," Journal of Monetary Economics, 51:1183-1216, 2004.

Beaudry, Paul and Franck Portier, "Stock Prices, News, and Economic Fluctuations," American Economic Review, 96:1293-1307, 2006.

Beaudry, Paul and Franck Portier, "When can changes in expectations cause business cycle fluctuations in neo-classical settings?," Journal of Economic Theory, 135:458-477, 2007.

Beaudry, Paul, and Eric van Wincoop, "The Intertemporal Elasticity of Substitution: An Exploration using a US Panel of State Data," Economica, 63:495-512, 1996.

Boyce, William E., and Richard C. DiPrima, Elementary Differential Equations and Boundary Value Problems, John Wiley \& Sons, New York, 1969.

Campbell, John Y., "Inspecting the mechanism: An analytical approach to the stochastic growth model," Journal of Monetary Economics, 33:463-506, 1997.

Christiano, Lawrence, Cosmin Ilut, Roberto Motto, and Massimo Rostagno, "Monetary policy and stock market boom-bust cycles," Working Paper Series 955, European Central Bank, 2008.

Comin, Diego A., Mark Gertler, and Ana Maria Santacreu, "Technology Innovation and Diffusion as Sources of Output and Asset Price Fluctuations," Harvard Business School 
Working Paper, 09:134, 2009.

Flóden, Martin, "Vintage Capital and Expectations Drive Business Cycles," CEPR working paper, 2009.

Greenwood, Jeremy, Zvi Hercowitz, Gregory W. Huffman, "Investment, Capacity Utilization, and the Real Business Cycle," American Economic Review, 78:402-417, 1988.

Gunn, Christopher, and Alok Johri, "News and Knowledge Capital," Review of Economic Dynamics, 14:92-101, 2011.

Huffman, Gregory and Mark Wynne, "The role of intratemporal adjustment costs in a multisector economy," Journal of Monetary Economics, 43:317-350, 1999.

Jaimovich, Nir, and Sergio Rebelo, "Can News About the Future Drive the Business Cycle?," American Economic Review, 99:1097-1118, 2009

Greenwood, Jeremy, Zvi Hercowitz, Gregory W. Huffman, "Investment, Capacity Utilization, and the Real Business Cycle," American Economic Review, 78:402-417, 1988.

Judd, Kenneth, "An Alternative to Steady-State Comparisons in Perfect Foresight Models," Economic Letters, 10:55-59, 1982.

Judd, Kenneth, "Short-Run Analysis of Fiscal Policy in a Simple Perfect Foresight Model," Journal of Political Economy, 93:298-319, 1985.

Khan, Hashmat and John Tsoukalas, "The Quantitative Importance of News Shocks in an Estimated DSGE Model," Journal of Money, Credit, and Banking, forthcoming, 2012.

King, Robert G., Charles I. Plosser and Sergio T. Rebelo, "Production, Growth and Business Cycles I. The Basic Neoclassical Model," Journal of Monetary Economics, 21: 195-232, 1988.

Leeper, Eric M., Todd Walker, and Shu-Chun (Susan) Yang, "Foresight and Information Flows," mimeo, Indiana University, 2012. 
Mulligan, Casey, "Capital, Interest, and Aggregate Intertemporal Substitution," NBER Working Paper 9373, 2002.

Nah, Seungho, "Financial Frictions, Intersectoral Adjustment Costs, and News-Driven Business Cycles," mimeo, The Ohio State University, 2009.

Qureshi, Hammad, "News Shocks and Learning-by-Doing," mimeo, The Ohio State University, 2009.

Schmitt-Grohe, Stephanie and Martin Uribe, "What's News in Business Cycles," Econometrica, forthcoming, 2012.

Sims, Christopher A., "Models and their Uses," American Journal of Agricultural Economics, 71:489-494, 1989.

Tenenbaum, Morris and Harry Pollard, Ordinary Differential Equations, Dover, 1985.

Tsai, Yi-Chan, "News Shocks and Costly Technology Adoption," mimeo, The University of Tokyo, 2009.

Wang, Pengfei, "Understanding Expectation-Driven Fluctuations-A Labor Market Approach," Journal of Money, Credit and Banking, 44:487-506, 2012.

Valles, Javier, "Aggregate Investment in a Business Cycle Model with Adjustment Costs," Journal of Economic Dynamics and Control, 21:1181-1198, 1997

Vissing-Jorgensen, Annette, and Orazio P. Attanasio, "Stock Market Participation, Intertemporal Substitution, and Risk Aversion," American Economic Review, 93:383-391, 2003. 
Table 1: Parameterization for computing impulse responses from various models

\begin{tabular}{cll}
\hline \hline Parameter & Value & Description \\
\hline \hline$\beta=\frac{1}{\rho+1}$ & 0.985 & Subjective discount factor (quarterly) \\
$\alpha$ & 0.33 & Labor share in production is $(1-\alpha)$ \\
$\delta$ & 0.025 & Depreciation rate of capital \\
$v$ & 1.8 & Degree of complementarity between $c$ and $i\left(>v_{c}=(1-\alpha)^{-1}\right)$ \\
$\sigma$ & 0.5 & Utility curvature \\
$\theta$ & 0.253 & Calibrated to match a steady state price of investment of 1 \\
$T$ & 4 & Quarters between news and actual technology increase \\
\hline & & Labor Externality \\
\hline$\sigma$ & 1 & Utility curvature - separable Utility \\
$\gamma_{N}$ & 0.332 & Degree of labor externality \\
$\psi_{I}$ & 20 & Investment adjustment cost \\
\hline & \multicolumn{2}{c}{ GHH preferences } \\
\hline$\omega$ & 0.5 & Utility curvature \\
$\frac{1}{\psi}$ & 100 & Frisch Elasticity \\
$\xi$ & 6.96 & Calibrated to match steady state labor of 0.3 \\
\hline & & Investment Adjustment Costs \\
\hline$\psi_{I}$ & 1 & Utility curvature - separable utility \\
\hline$\sigma$ & 10 & Investment adjustment cost \\
\hline$\tau$ & 1 & Forward Compatible Investment \\
\hline \hline
\end{tabular}



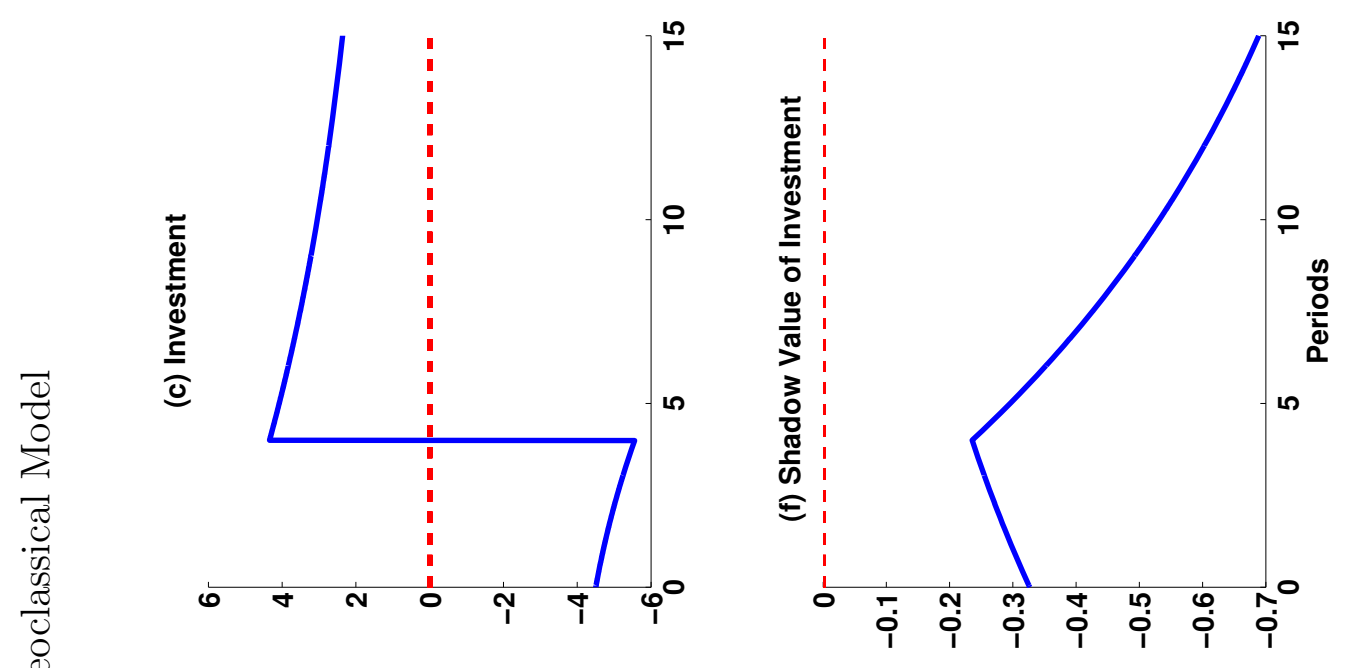

Z

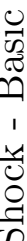

告

萬
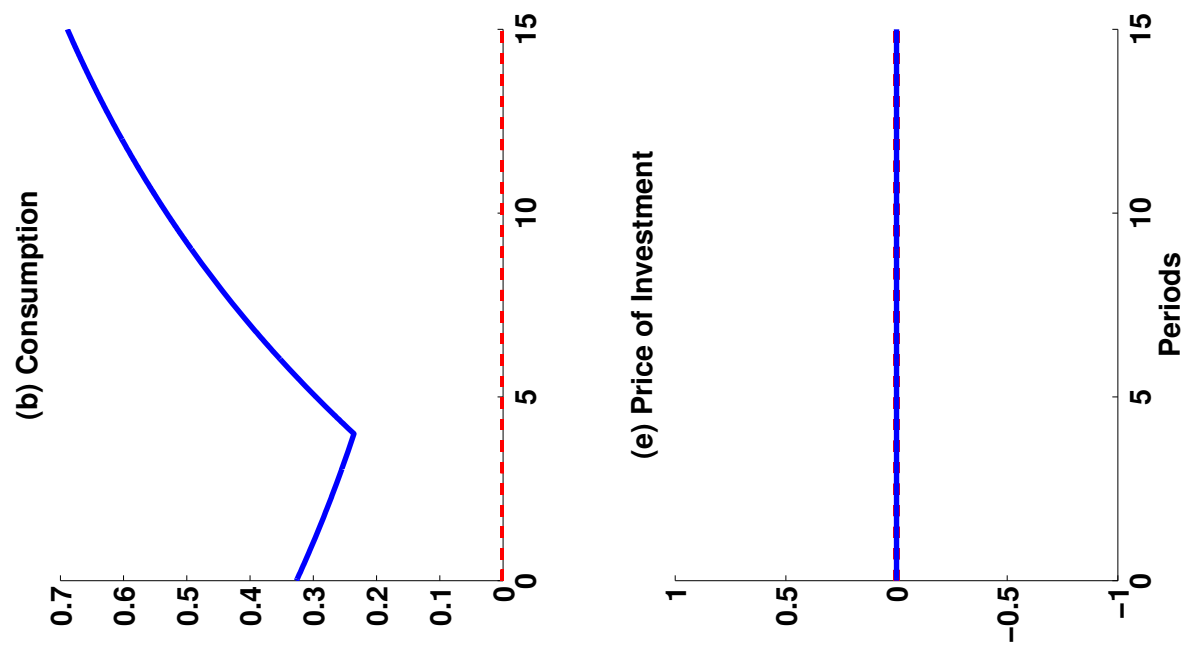

告
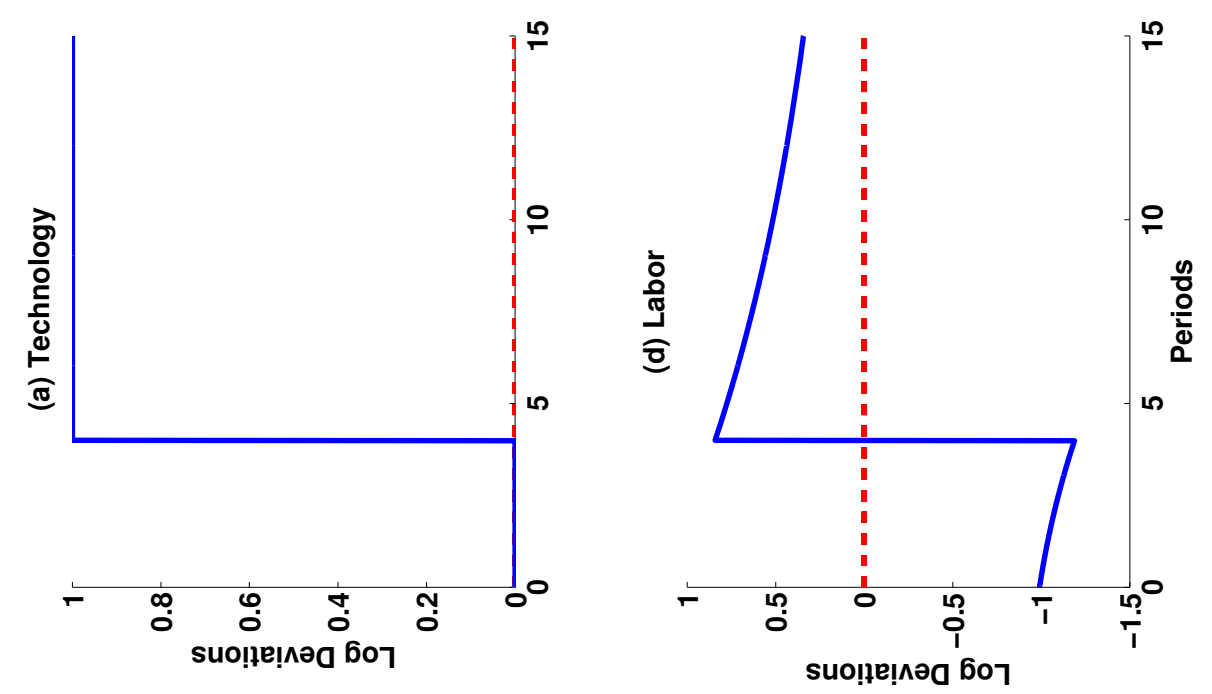
Figure 2: Production frontier between consumption and investment as $\nu$ changes.

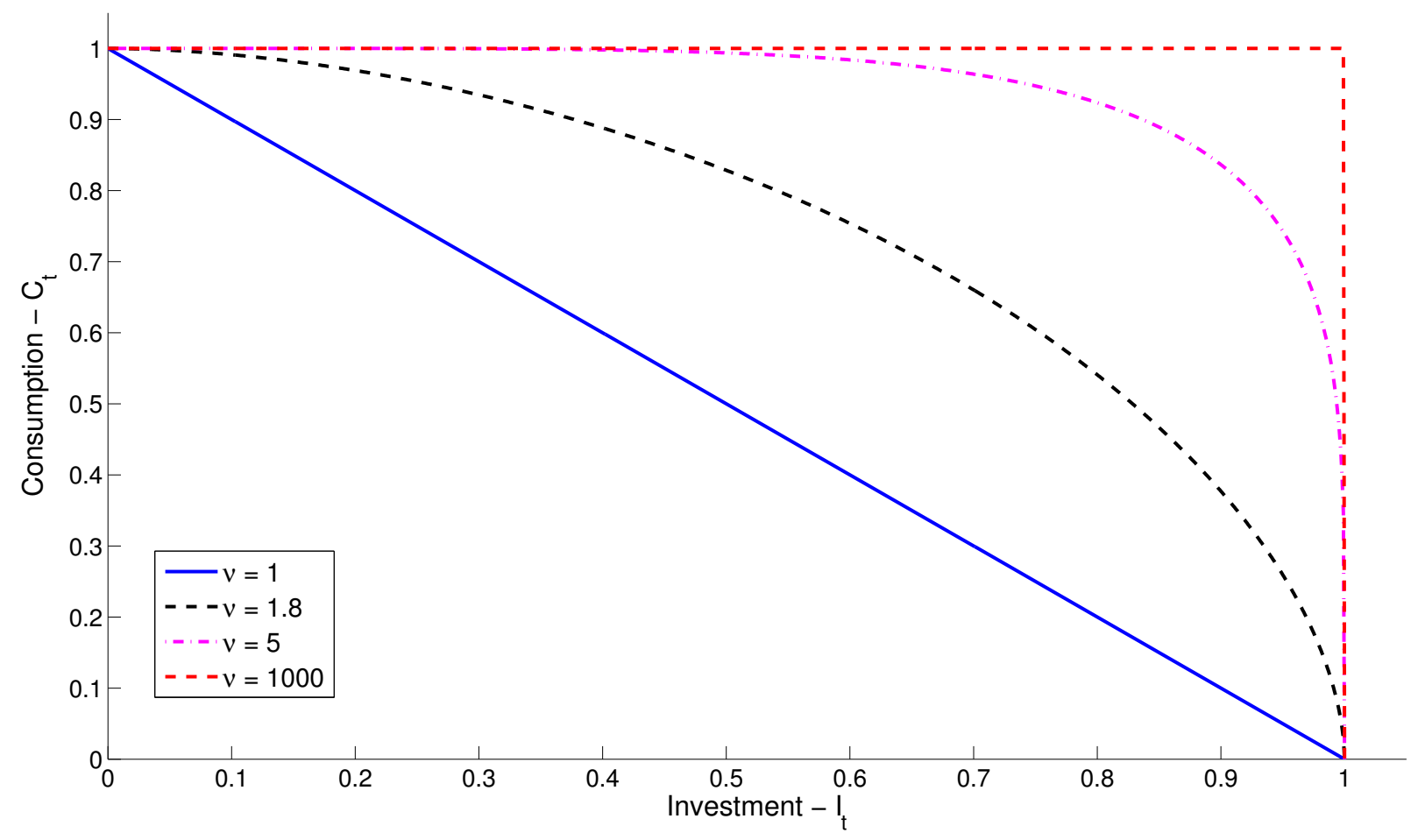

Note: $\theta=1 / 2$ and the fixed output level is equal to $(1 / 2)^{1 / \nu}$. 


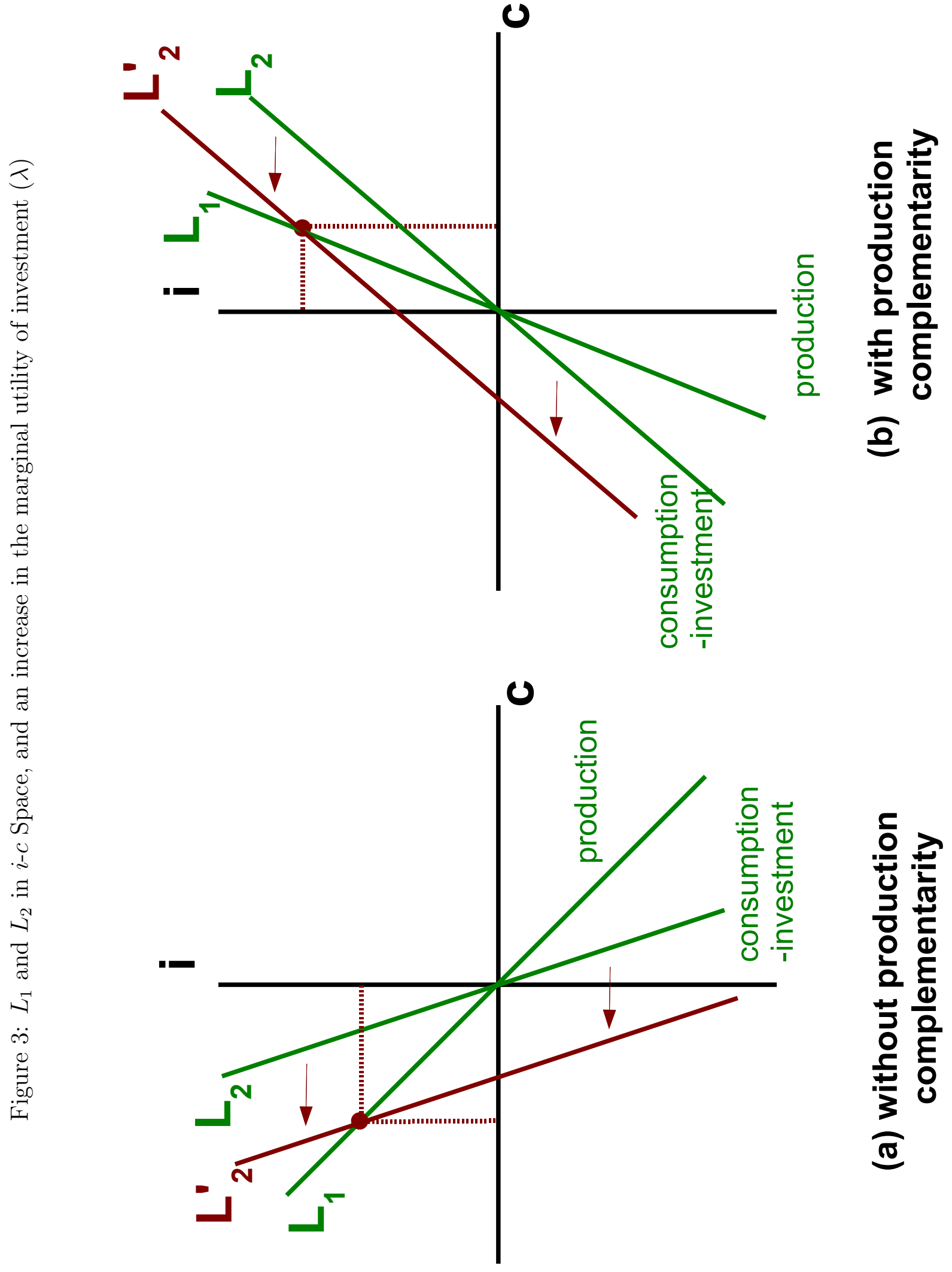




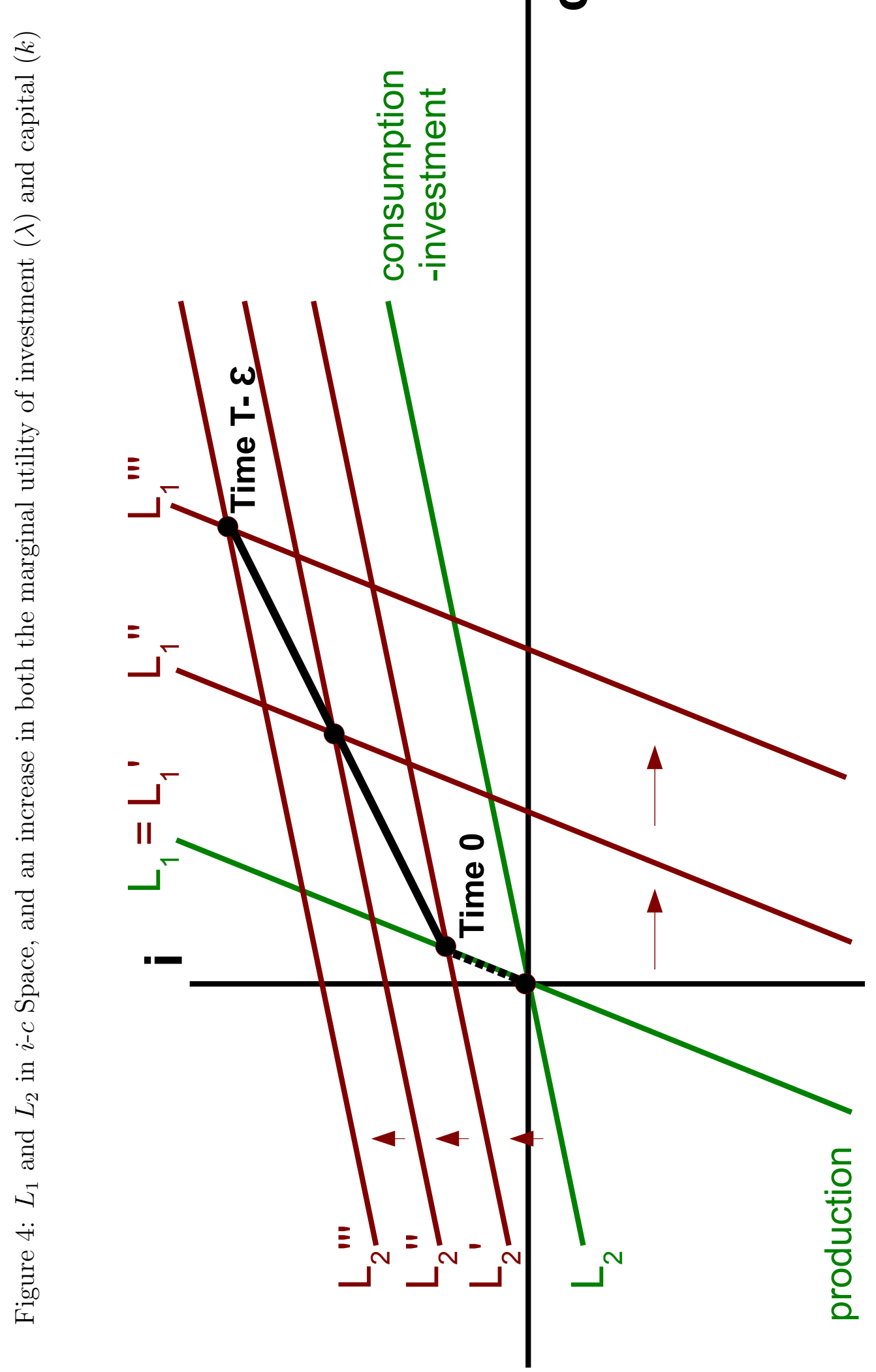


Figure 5: Phase diagrams in $(k, \lambda)$ space for a positive news shock

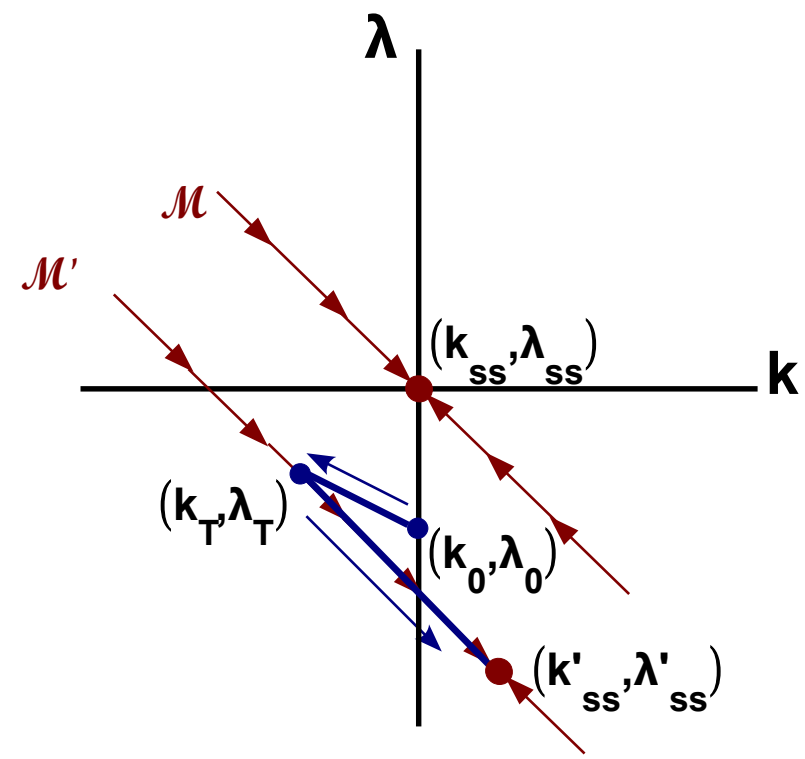

(a) Standard Production, $\sigma=1$

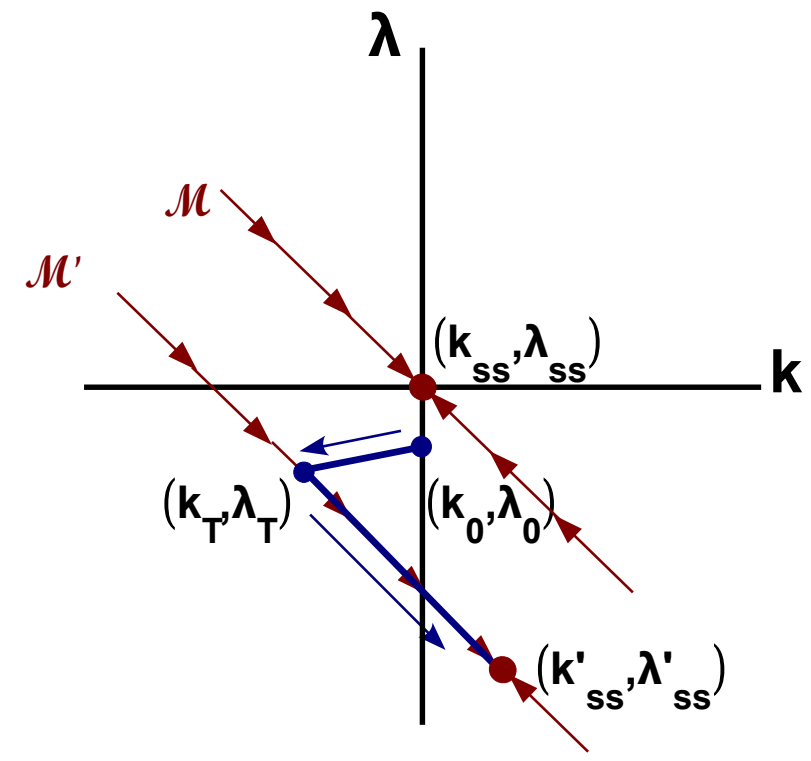

(b) Production Complementarities, High $\sigma$
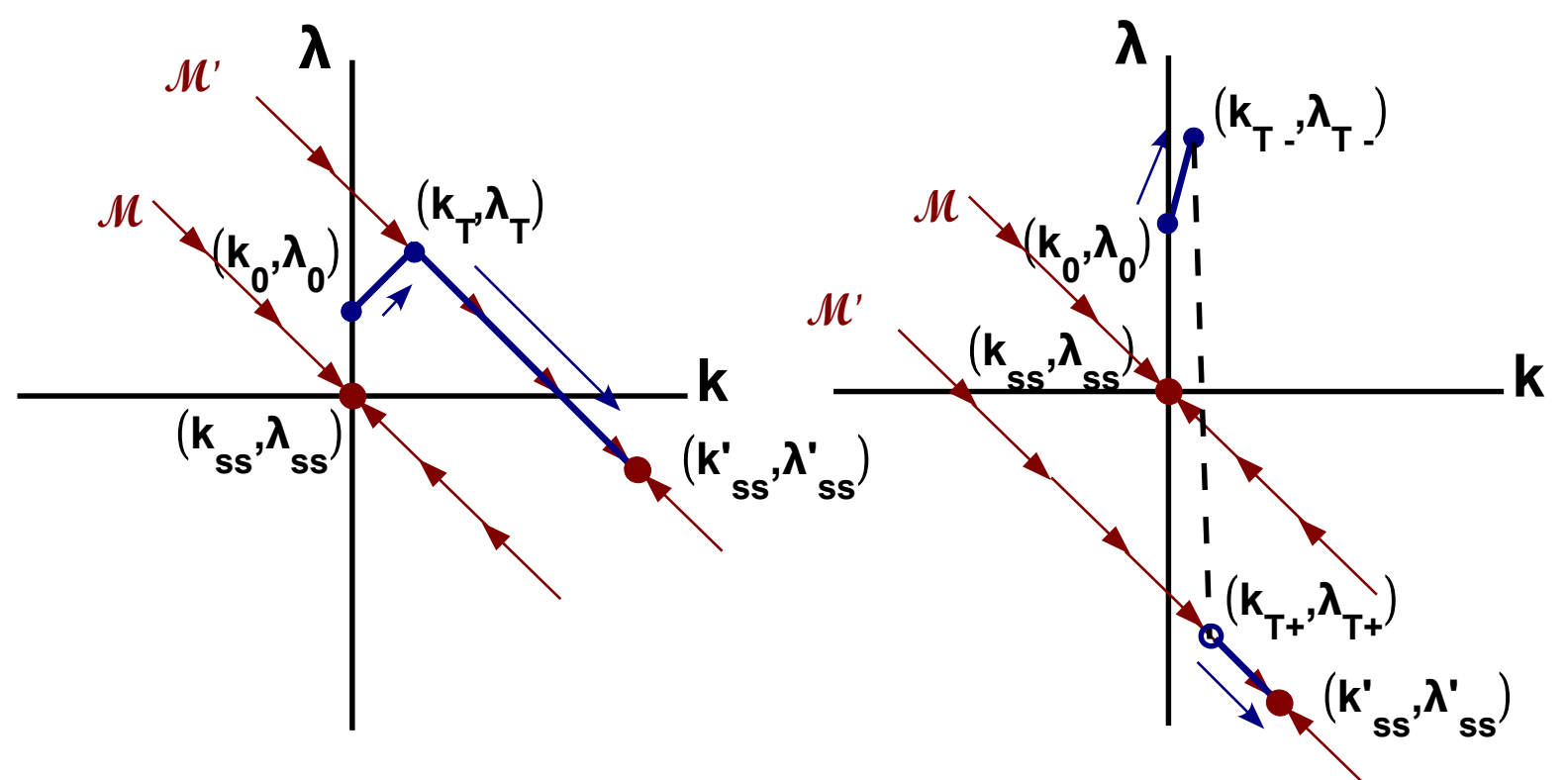

(c) Production Complementarities, Low $\sigma$

(d) Production Complementarities, Partial Forward Compatibility 






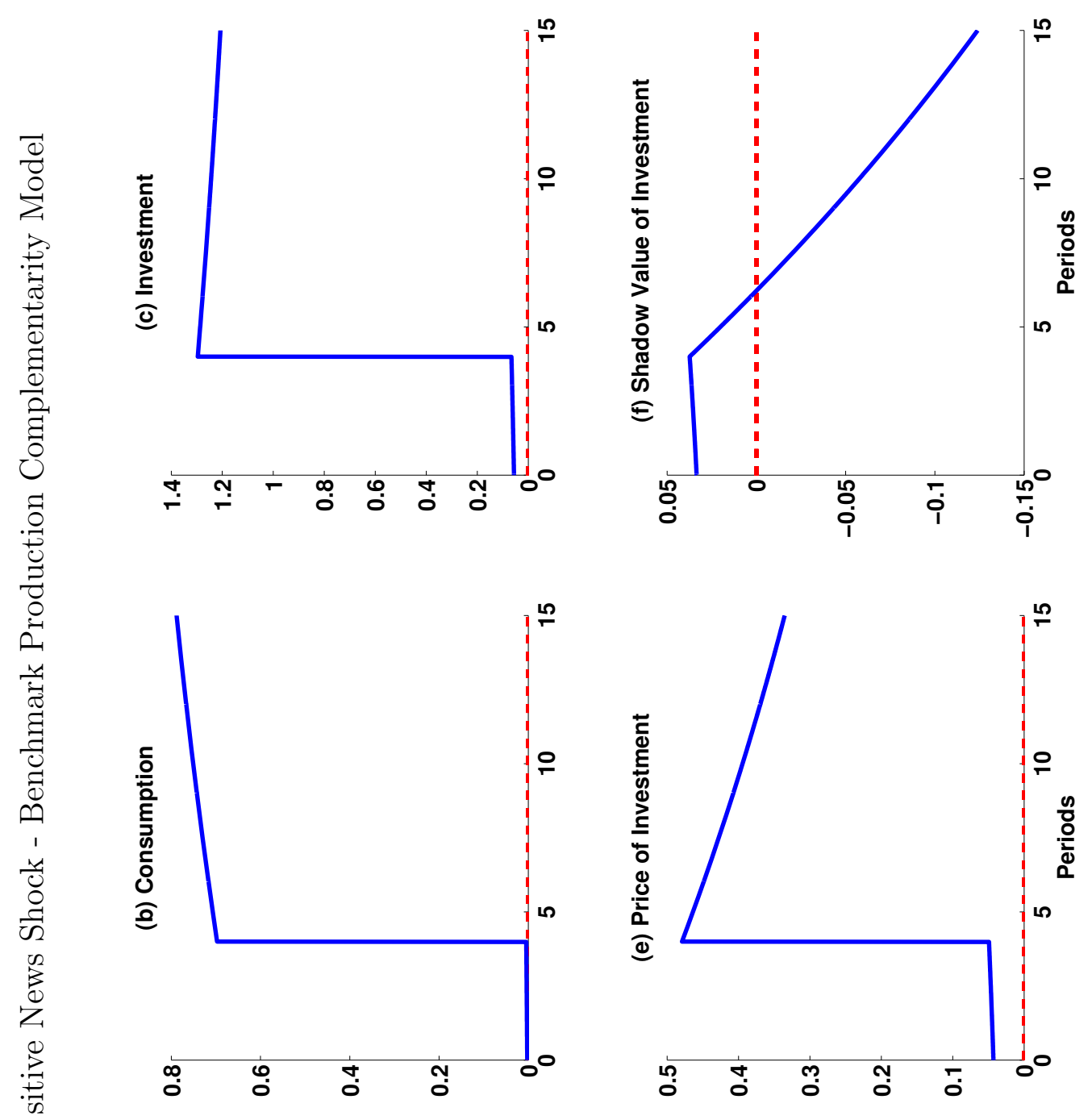

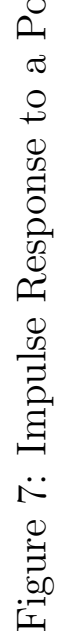
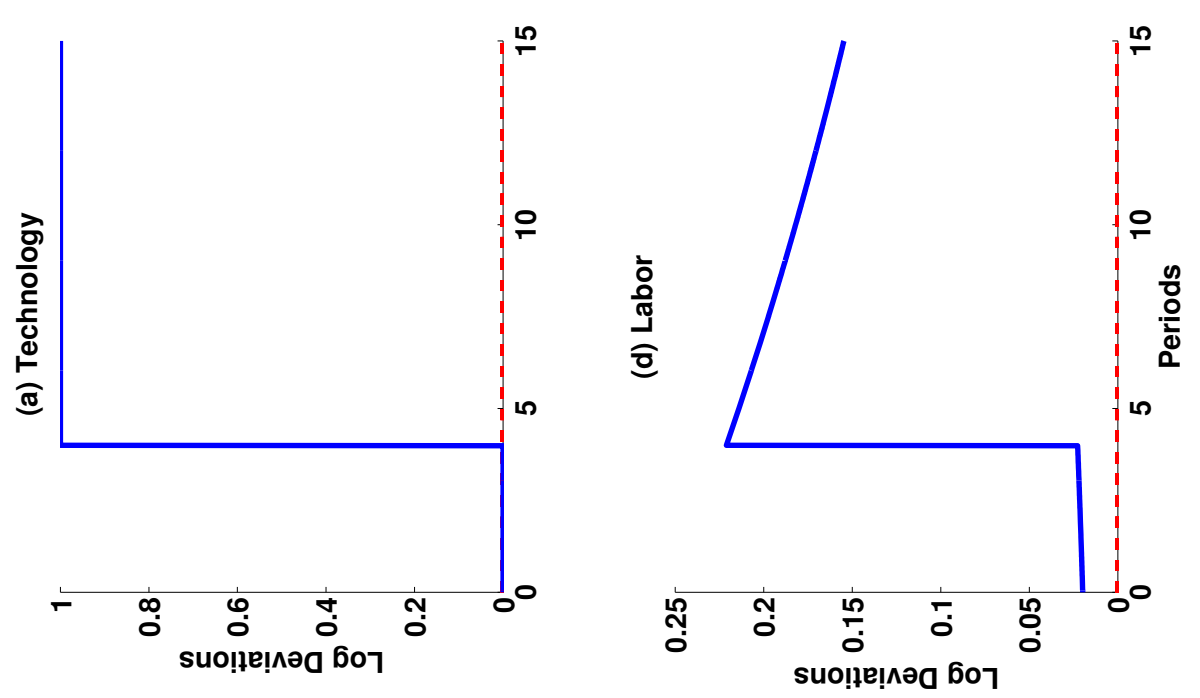


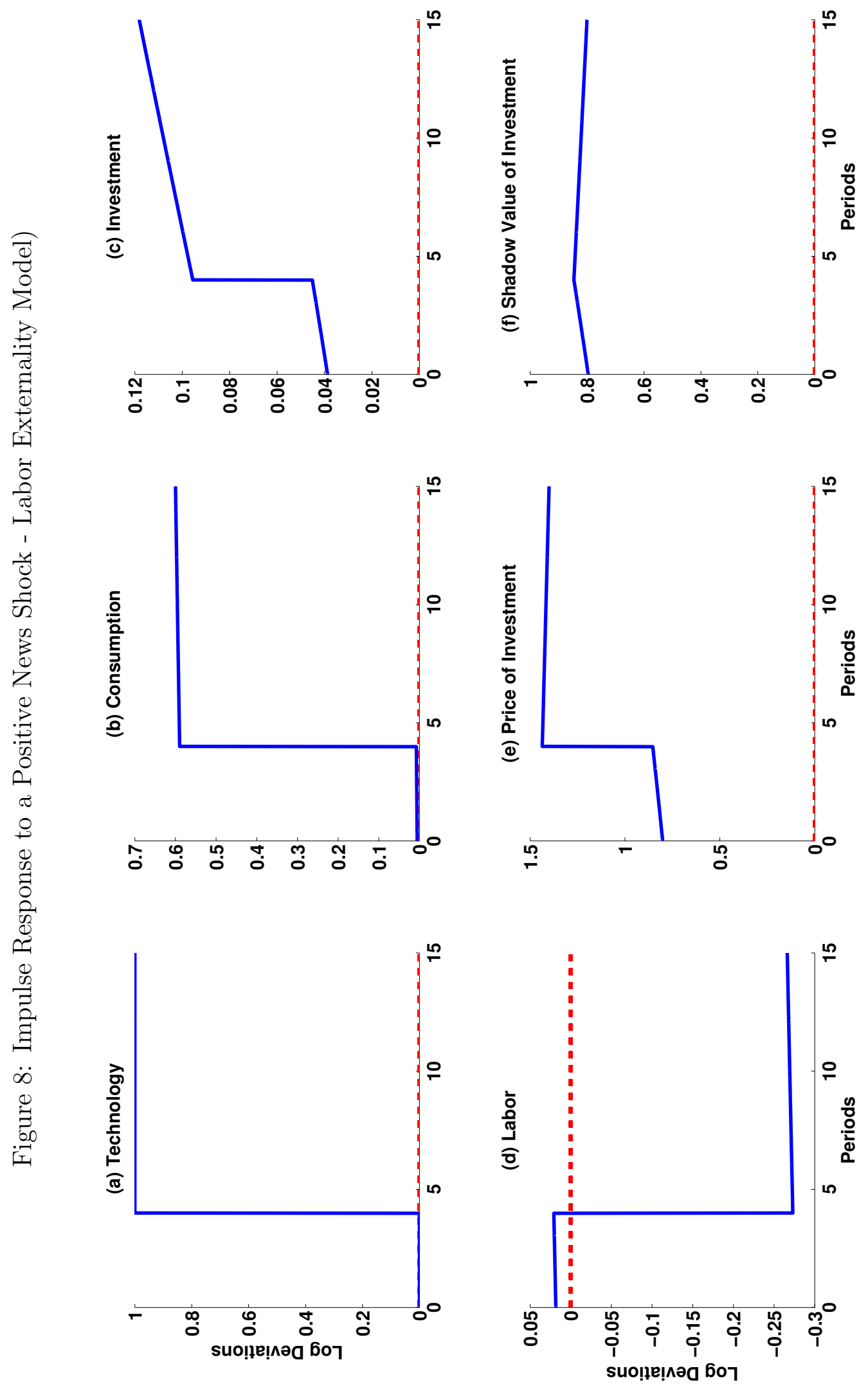




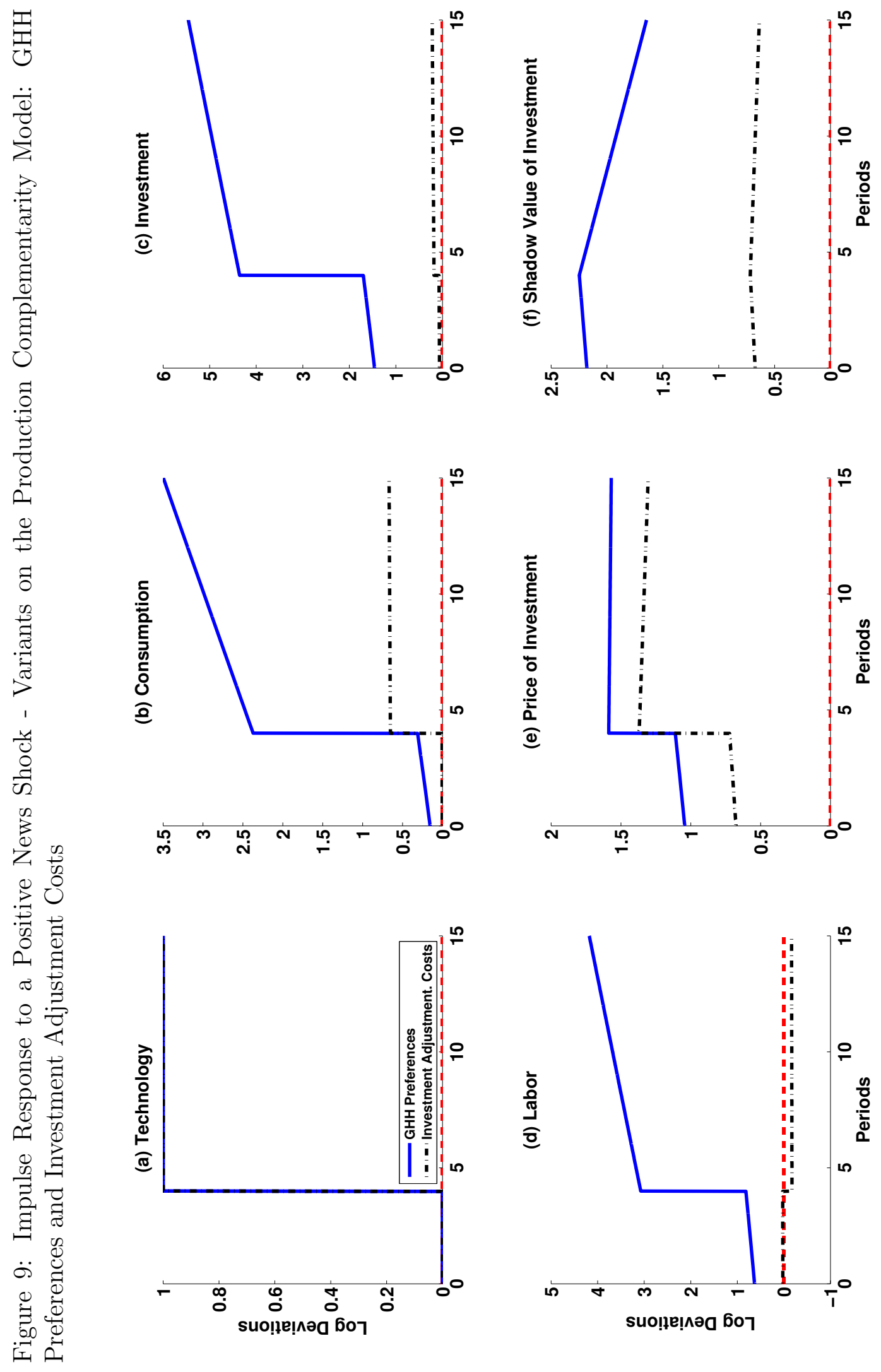


Figure 10: Efficiency of Different Types of Capital

(a) capital efficiency of post- $T$ investment

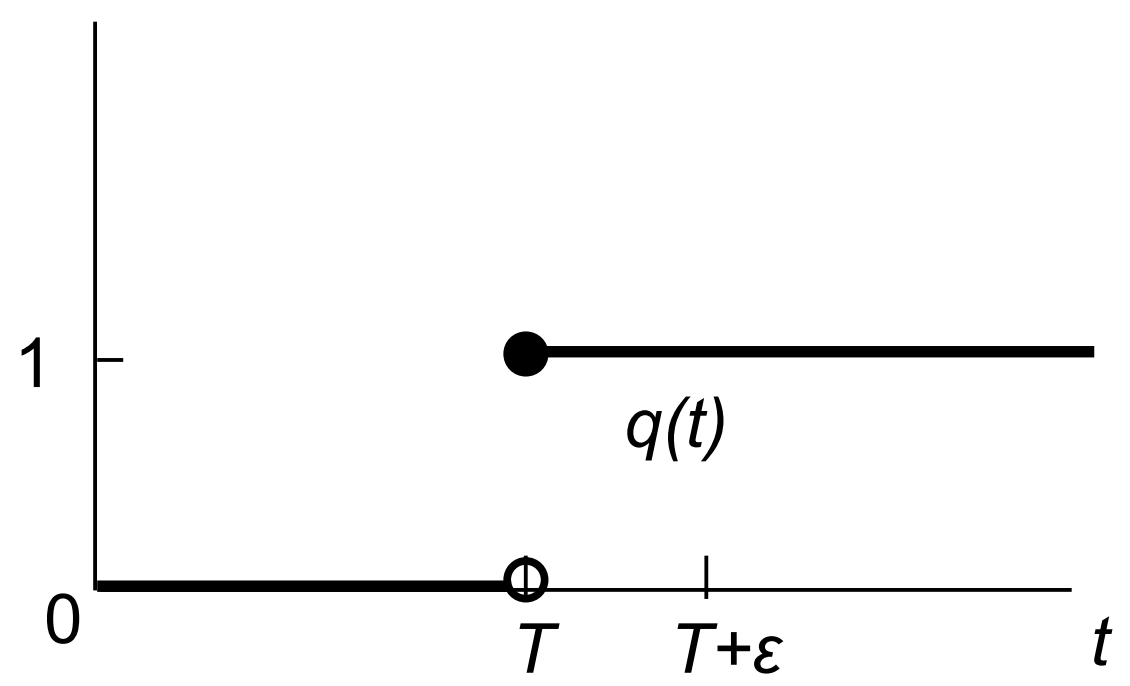

(b) change in capital efficiency of investment (done in preparatory phase)

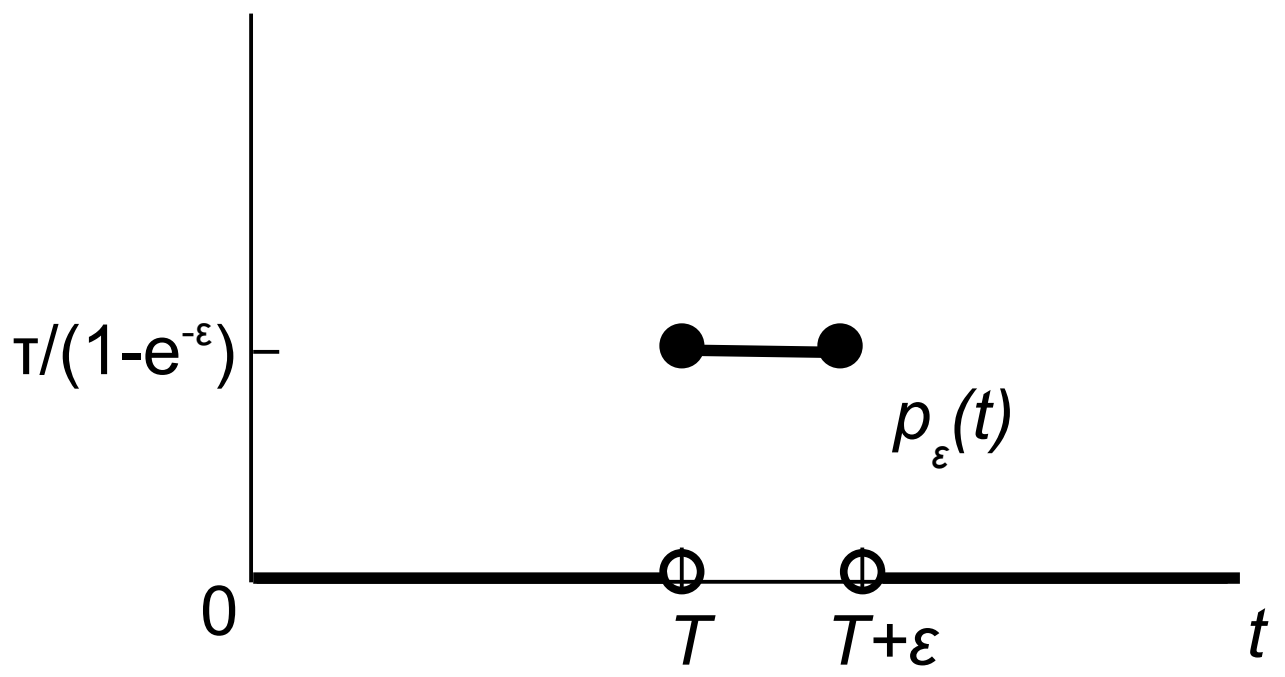



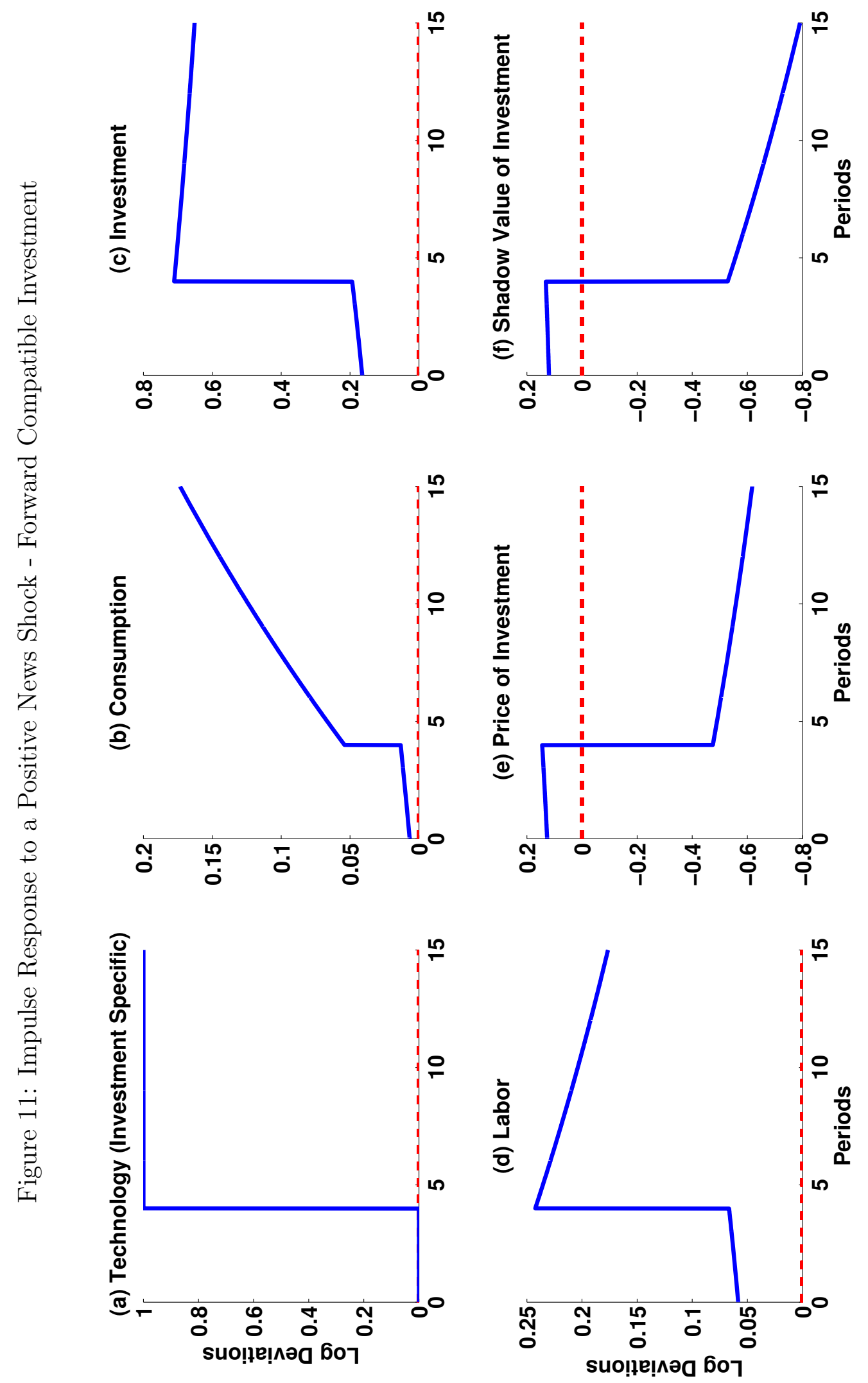

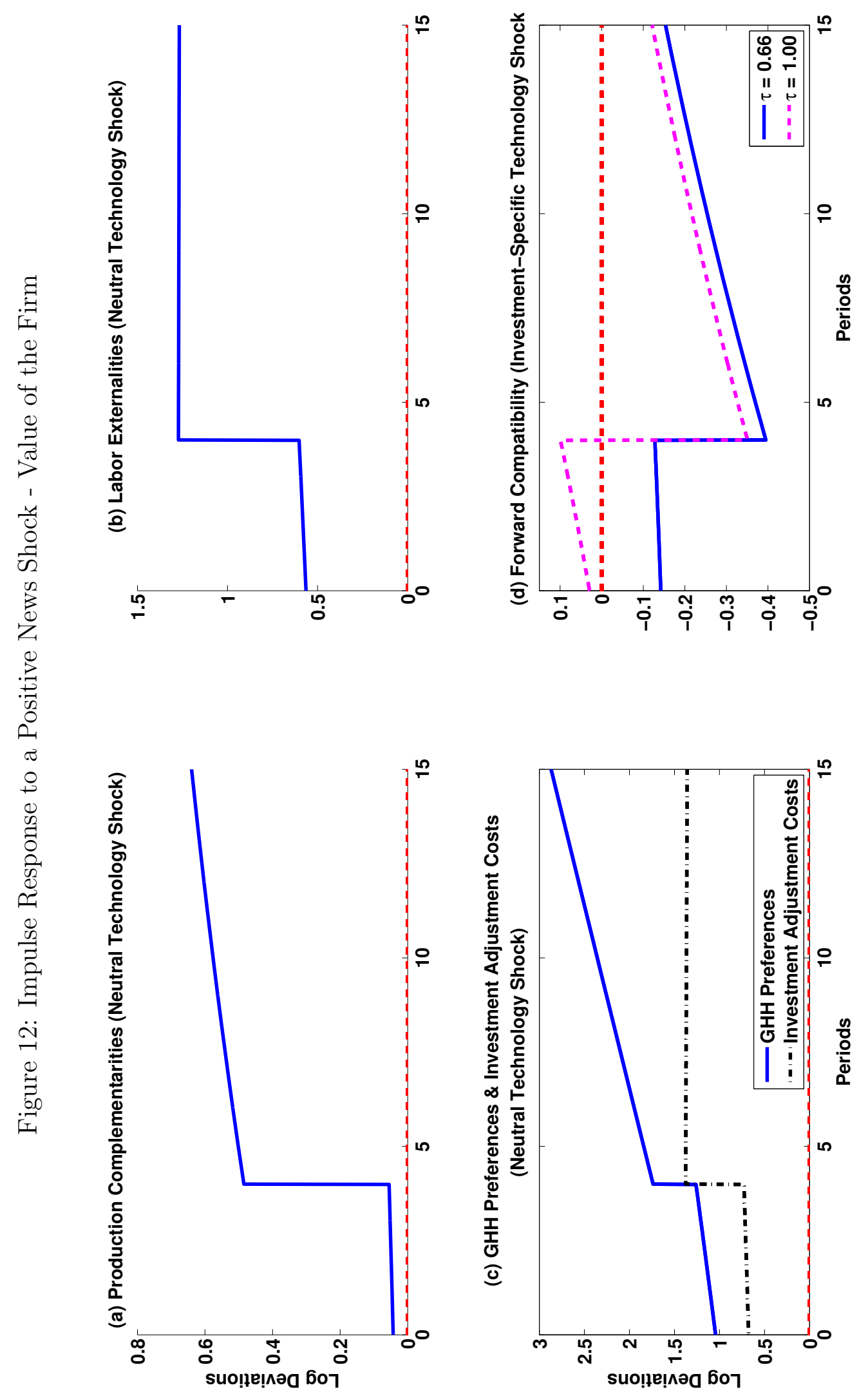


\section{A Lemmas and Theorems}

Proof of Lemma 1. Substituting (9) into (12):

$$
\begin{aligned}
\alpha k+(1-\alpha) a & =\left(1-(1-v(1-\alpha)) s_{I}\right) c+(1-v(1-\alpha)) s_{I} i \\
& =\left(1-\phi_{I}^{P C}\right) c+\phi_{I}^{P C} i
\end{aligned}
$$

Here $\phi_{I}^{P C}=(1-v(1-\alpha)) s_{I}$.

$k(0)=0$ and $a(0)=0$, therefore $(42)$ can be written as:

$$
0=\left(1-\phi_{I}^{P C}\right) c(0)+\phi_{I}^{P C} i(0)
$$

First, if $v(1-\alpha)>1$, then $\phi_{I}^{P C}<0 \&\left(1-\phi_{I}^{P C}\right)>0$. Therefore, if $v(1-\alpha)>1$ and $c(0)$ increases then for (43) to hold $i(0)$ must also increase.

Further, if $v(1-\alpha)>1$, then $\left(1-\phi_{I}^{P C}\right)=\left(-\phi_{I}^{P C}+1\right)>-\phi_{I}$. Therefore, if $c(0)$ increases, then for (43) to hold $i(0)$ must increase by a larger magnitude than $c(0)$, this implies $(i(0)-c(0))$ increases when $c(0)$ increases, which in turn due to (9) implies that $n(0)$ must increase.

Therefore, if $v(1-\alpha)>1$, then consumption, investment, and hours will comove at time zero.

Second, if $v(1-\alpha)<1$, then $\phi_{I}^{P C} \in(0,1)$ and $\left(1-\phi_{I}^{P C}\right)>0$. Therefore, if $c(0)$ increases then for (43) to hold $i(0)$ must decrease. Therefore, if $v(1-\alpha)<1$, then consumption, investment and hours will not comove at time zero.

Proof of Lemma 2. Substituting (9) into (10):

$$
\gamma_{I}^{P C} i-\left(\sigma+\gamma_{I}^{P C}\right) c=\lambda
$$

Here $\gamma_{I}^{P C}=(v-1)-\left(v(1-\alpha)(1-\sigma) s_{I}\right) /\left(1-s_{I}\right)$. 
Further, substituting (42) into (44) and solving for $c$ at time 0 leads to:

$$
c(0)=\frac{-\phi_{I}^{P C}}{\gamma_{I}^{P C}+\phi_{I}^{P C} \sigma} \lambda(0)
$$

First, from the proof of lemma 1 we know that $-\phi_{I}^{P C}>0$ if $v(1-\alpha)>1$. Also, $\gamma_{I}^{P C}+$ $\phi_{I}^{P C} \sigma>0$ if $v(1-\alpha)>1 .^{35}$ From equation (45) if $\lambda(0)>0$ then $c(0)$ will increase. If $c(0)>0$ then from the proof of lemma 1 we know that both $i(0)$ and $n(0)$ will also increase.

As a result, if $v(1-\alpha)>1$ and $\lambda(0)>0$, then consumption, investment, and labor hours will comove procyclically at time zero in response to a news shock about technology in time $T>0$.

Second, by Lemma 1 we also know that if $v(1-\alpha)<1$ and $\lambda(0)>0$, then consumption and investment will not comove at time zero.

Proof of Lemma 3. Solving (42) and (44) simultaneously for the values of $c$ and $i$ :

$$
\begin{gathered}
c=\tau_{c, k}^{P C} k+\tau_{c, \lambda}^{P C} \lambda+\tau_{c, a}^{P C} a \\
i=\tau_{i, k}^{P C} k+\tau_{i, \lambda}^{P C} \lambda+\tau_{i, a}^{P C} a \\
n=\tau_{n, k}^{P C} k+\tau_{n, \lambda}^{P C} \lambda+\tau_{n, a}^{P C} a
\end{gathered}
$$

Here $\tau_{c, k}^{P C}, \tau_{c, \lambda}^{P C} \tau_{i, k}^{P C}, \tau_{i, \lambda}^{P C}, \tau_{n, k}^{P C}$, and $\tau_{n, \lambda}^{P C}$ are all positive. ${ }^{36}$

It follows directly that if $\dot{\lambda} \geq 0$ and $\dot{k} \geq 0 \forall t<T$ then $\dot{c} \geq 0, \dot{i} \geq 0$, and $\dot{n} \geq 0$ for all $t<T$. Again, remember for $\forall t<T, a(t)=0$.

Proof of Lemma 4. Recall $k(0)=0$. As a result, the time derivatives of the $k(t)$ and $\lambda(t)$

\footnotetext{
${ }^{35}$ For the proof see Lemma B.3 in Appendix B (Supplementary Appendix).

${ }^{36}$ For the proof see Lemma B.4 in Appendix B (Supplementary Appendix).
} 
paths for all $t<T$ :

$$
\begin{gathered}
\dot{k}(t)=\frac{\Gamma_{k, \lambda}^{P C}\left(\mu_{2}^{P C} e^{\mu_{2}^{P C} t}-\mu_{1}^{P C} e^{\mu_{1}^{P C} t}\right)}{\mu_{2}^{P C}-\mu_{1}^{P C}} \lambda(0) \\
\dot{\lambda}(t)=\left[\frac{\left(\mu_{2}^{P C}-\Gamma_{k, k}^{P C}\right)}{\mu_{2}^{P C}-\mu_{1}^{P C}} \mu_{2}^{P C} e^{\mu_{2}^{P C} t}-\frac{\left(\mu_{1}^{P C}-\Gamma_{k, k}^{P C}\right)}{\mu_{2}^{P C}-\mu_{1}^{P C}} \mu_{1}^{P C} e^{\mu_{1}^{P C} t}\right] \lambda(0)
\end{gathered}
$$

First, for $0 \leq t<T:\left(\Gamma_{k, \lambda}^{P C}\left(\mu_{2}^{P C} e^{\mu_{2}^{P C} t}-\mu_{1}^{P C} e^{\mu_{1}^{P C} t}\right)\right) /\left(\mu_{2}^{P C}-\mu_{1}^{P C}\right)$ is positive as $\Gamma_{k, \lambda}^{P C}>0$, 37 and we know that $\mu_{2}^{P C}>0$ and $\mu_{1}^{P C}<0$. Therefore the $\operatorname{sign}(\dot{k}(t))=\operatorname{sign}\left(\lambda_{0}\right)$.

Second, for the $\dot{\lambda}$ equation: $\left(\mu_{2}^{P C}-\Gamma_{k, k}^{P C}\right) \mu_{2}^{P C} e^{\mu_{2}^{P C} t} /\left(\mu_{2}^{P C}-\mu_{1}^{P C}\right)$ is positive because $\mu_{2}^{P C}-$ $\Gamma_{k, k}^{P C}=\Gamma_{\lambda, \lambda}^{P C}-\mu_{1}^{P C}, 38$ and we know $\mu_{1}^{P C}<0$ and $\Gamma_{\lambda, \lambda}^{P C}>0 .{ }^{39}$

$\left(\mu_{1}^{P C}-\Gamma_{k, k}^{P C}\right) \mu_{1}^{P C} e^{\mu_{1}^{P C} t} /\left(\mu_{2}^{P C}-\mu_{1}^{P C}\right)$ may be either positive or negative. If $\mu_{1}^{P C}-\Gamma_{k, k}^{P C}>0$, then the second term on the right-hand side is positive. In this case, $\dot{\lambda}(t)>0$. However, if $\mu_{1}^{P C}-\Gamma_{k, k}^{P C}<0$, then $\left(\mu_{1}^{P C}-\Gamma_{k, k}^{P C}\right) \mu_{1}^{P C} e^{\mu_{1}^{P C} t} /\left(\mu_{2}^{P C}-\mu_{1}^{P C}\right)$ is negative. In this case, we must show that $\left(\mu_{2}^{P C}-\Gamma_{k, k}^{P C}\right) \mu_{2}^{P C} e^{\mu_{2}^{P C} t} /\left(\mu_{2}^{P C}-\mu_{1}^{P C}\right)$ is larger than $\left(\mu_{1}^{P C}-\Gamma_{k, k}^{P C}\right) \mu_{1}^{P C} e^{\mu_{1}^{P C} t} /\left(\mu_{2}^{P C}-\mu_{1}^{P C}\right)$ in order that $\dot{\lambda}(t)>0$. Because $\mu_{2}^{P C}>0>\mu_{1}^{P C}$, in this second case, the smallest value for $\dot{\lambda}(t)$ occurs at $t=0$.

$$
\begin{aligned}
\dot{\lambda}(0) & =\frac{\lambda(0)}{\mu_{2}^{P C}-\mu_{1}^{P C}}\left[\mu_{2}^{P C}\left(\mu_{2}^{P C}-\Gamma_{k, k}^{P C}\right)-\mu_{1}^{P C}\left(\mu_{1}^{P C}-\Gamma_{k, k}^{P C}\right)\right] \\
& =\lambda_{0}\left[\mu_{2}^{P C}+\mu_{1}^{P C}-\Gamma_{k, k}^{P C}\right] \\
& =\lambda_{0}\left[\Gamma_{k, k}^{P C}+\Gamma_{\lambda, \lambda}^{P C}-\Gamma_{k, k}^{P C}\right] \\
& =\lambda_{0} \Gamma_{\lambda, \lambda}^{P C}
\end{aligned}
$$

As $\Gamma_{\lambda, \lambda}^{P C}>0$, this establishes that $\operatorname{sign}(\dot{\lambda}(t))=\operatorname{sign}\left(\lambda_{0}\right)$.

\footnotetext{
${ }^{37}$ For the proof see Lemma B.5 in Appendix B (Supplementary Appendix).

${ }^{38}$ This follows because $\operatorname{tr}\left(\Gamma^{P C}\right)=\mu_{1}^{P C}+\mu_{2}^{P C}$.

${ }^{39}$ For the proof see Lemma B.5 in Appendix B (Supplementary Appendix).
} 
Proof of Lemma 5. Recall $\mu_{2}^{P C}>0$ and

$$
k(t)=\left\{\begin{array}{cc}
\frac{\Gamma_{k, \lambda}^{P C} \lambda(0)+\left(\mu_{1}^{P C}-\Gamma_{\lambda, \lambda}^{P C}\right) k(0)}{\mu_{1}^{P C}-\mu_{2}^{P C}} e^{\mu_{1}^{P C} t}+\frac{\Gamma_{k, \lambda}^{P C} \lambda(0)+\left(\mu_{2}^{P C}-\Gamma_{\lambda, \lambda}^{P C}\right) k(0)}{\mu_{2}^{P C}-\mu_{1}^{P C}} e^{\mu_{2}^{P C} t} & \text { for } t \in[0, T) \\
\frac{\Gamma_{k, \lambda}^{P C} \lambda(0)+\left(\mu_{1}^{P C}-\Gamma_{\lambda, \lambda}^{P C}\right) k(0)}{\mu_{1}^{P C}-\mu_{2}^{P C}} e^{\mu_{1}^{P C} t}+\frac{\Gamma_{k, \lambda}^{P C} b_{\lambda, a}^{P C}-\Gamma_{\lambda, \lambda}^{P C} b_{k, a}^{P C}}{\mu_{1}^{P C} \mu_{2}^{P C}}+\frac{\Gamma_{k, \lambda}^{P C} b_{\lambda, a}^{P C}+\left(\mu_{1}^{P C}-\Gamma_{\lambda, \lambda}^{P C}\right) b_{k, a}^{P C}}{\mu_{1}^{P C}\left(\mu_{1}^{P C}-\mu_{2}^{P C}\right)} e^{\mu_{1}^{P C}(t-T)} & t \geq T
\end{array}\right.
$$

Then as $k(0)=0$ a non-explosive path for $[\lambda k]^{\prime}$ requires that we choose $\lambda(0)$ such that the terms involving the explosive root $\mu_{2}^{P C}$ in the exponential are 'zeroed out' for all $t>T$. Otherwise the path for $k(t)$ will be explosive. This imposes the following restriction on $\lambda(0)$ :

$$
\left(\frac{\Gamma_{k, \lambda}^{P C}}{\mu_{2}^{P C}-\mu_{1}^{P C}}\right) \lambda_{0}=-\frac{\Gamma_{k, \lambda}^{P C} b_{\lambda, a}^{P C}+\left(\mu_{2}^{P C}-\Gamma_{\lambda, \lambda}^{P C}\right) b_{k, a}^{P C}}{\mu_{2}^{P C}\left(\mu_{2}^{P C}-\mu_{1}^{P C}\right)} e^{-\mu_{2}^{P C} T}
$$

This can be re-written as:

$$
\lambda_{0}=-\left[\frac{\Gamma_{k, \lambda}^{P C} b_{\lambda, a}^{P C}+\left(\mu_{2}^{P C}-\Gamma_{\lambda, \lambda}^{P C}\right) b_{k, a}^{P C}}{\Gamma_{k, \lambda}^{P C} \mu_{2}^{P C}}\right] e^{-\mu_{2}^{P C} T}
$$

Because $\Gamma_{k, \lambda}^{P C}>0, \lambda(0)>0$ if and only if $\Gamma_{k, \lambda}^{P C} b_{\lambda, a}^{P C}+\left(\mu_{2}-\Gamma_{\lambda, \lambda}^{P C}\right) b_{k, a}^{P C}<0$. Also, $\Gamma_{k, \lambda}^{P C} b_{\lambda, a}^{P C}+$ $\left(\mu_{2}-\Gamma_{\lambda, \lambda}^{P C}\right) b_{k, a}^{P C}<0$ algebraically simplifies to $\mu_{2}^{P C}<(\rho+(1-\alpha) \delta) v /\left(\gamma_{I}+\sigma\right)$.

Proof of Theorem 1. $\Leftarrow$. If $v(1-\alpha)>1$ and $\mu_{2}^{P C}<(\rho+(1-\alpha) \delta) v /\left(\gamma_{I}^{P C}+\sigma\right)$, then a technology news shock is procyclical. Lemmas 2 and 5 prove the procyclical comovement at $t=0$, while Lemmas 3, 4 and 5 establish the procyclical comovement for $0<t<T$.

$\Rightarrow$. If $v(1-\alpha)<1$ or $\mu_{2}^{P C}<(\rho+(1-\alpha) \delta) v /\left(\gamma_{I}^{P C}+\sigma\right)$, then a technology news shock is not procyclical. This follow trivially from Lemma 2 , as the procyclical comovement will not occur at time $t=0$ if either of the above conditions are not met.

Proof of Lemma 6. The condition $\mu_{2}^{P C}<(\rho+(1-\alpha) \delta) v /\left(\gamma_{I}^{P C}+\sigma\right)$ can be rewritten implicitly as $\sigma<\sigma^{*}$. As $\delta \rightarrow 0$ we have $\sigma^{*} \rightarrow 1$. The above lemma thus follows directly from Theorem 1. 
Proof of Lemma 7. Substituting (22) into (12):

$$
\begin{aligned}
\alpha k+(1-\alpha) a & =\left(1-\left(1-\frac{1-\alpha}{1-\gamma_{N}}\right) s_{I}\right) c+\left(1-\frac{1-\alpha}{1-\gamma_{N}}\right) s_{I} i \\
& =\left(1-\phi_{I}^{L E}\right) c+\phi_{I}^{L E} i
\end{aligned}
$$

Here $\phi_{I}^{L E}=\left(1-\frac{1-\alpha}{1-\gamma_{\bar{N}}}\right) s_{I}$.

$k(0)=0$ and $a(0)=0$, therefore $(50)$ can be written as:

$$
0=\left(1-\phi_{I}^{L E}\right) c(0)+\phi_{I}^{L E} i(0)
$$

First, if $\gamma_{N}>\alpha$, then $\phi_{I}^{L E}<0 \&\left(1-\phi_{I}^{L E}\right)>0$. Therefore, if $\gamma_{N}>\alpha$ and $c(0)$ increases then for (51) to hold $i(0)$ must also increase.

Further, if $\gamma_{N}>\alpha$, then $\left(1-\phi_{I}^{L E}\right)=\left(-\phi_{I}^{L E}+1\right)>-\phi_{I}^{L E}$. Therefore, if $c(0)$ increases, then for (51) to hold $i(0)$ must increase by a larger magnitude than $c(0)$, this implies $(i(0)-c(0))$ increases when $c(0)$ increases, which in turn due to (22) implies that $n(0)$ must increase.

Therefore, if $\gamma_{N}>\alpha$, then consumption, investment, and hours will comove at time zero.

Second, if $\gamma_{N}<\alpha$, then $\phi_{I}^{L E}>0$ and $\left(1-\phi_{I}^{L E}\right)>0$. Therefore, if $c(0)$ increases then for (51) to hold $i(0)$ must decrease. Therefore, if $\gamma_{N}<\alpha$, then consumption, investment and hours will not comove at time zero.

Proof of Lemma 8. For a stable solution to exist one eigenvalue of $\Gamma^{L E}$ should be positive and the other negative. The product of the eigenvalues is given by the determinant of the $\Gamma^{L E}$ matrix.

$$
\operatorname{det}\left(\Gamma^{L E}\right)=\frac{-\delta(\rho+\delta)}{\left(\phi_{I}^{L E} \sigma+\gamma_{I}^{L E}+\psi_{I}\left(1-\phi_{I}^{L E}\right)\right)}\left[\left(1-s_{I}\right)(1-\alpha)\right]
$$

First, if $\psi_{I}>\psi_{I}^{+}=-\frac{\gamma_{I}^{L E}+\phi_{I}^{L E} \sigma}{1-\phi_{I}^{L E}}$ then the product of the eigenvalues is negative and it follows that the eigenvalues have opposite signs. Further, it can be shown that $\operatorname{tr}\left(\Gamma^{L E}\right)=\rho$ which 
gives the sum of the two eigenvalues.

Second, if $\psi_{I}<\psi_{I}^{+}=-\frac{\gamma_{I}^{L E}+\phi_{I}^{L E} \sigma}{1-\phi_{I}^{L E}}$ then the product of the eigenvalues is positive and with $\operatorname{tr}\left(\Gamma^{L E}\right)=\rho$, which gives the sum of the two eigenvalues, it follows that the eigenvalues are both positive.

Proof of Lemma 9. Recall $\mu_{2}^{L E}>0$. Also, $\Gamma_{k, \lambda}^{L E}>0 .{ }^{40}$ For a stable solution we need:

$$
\lambda_{0}=-\left[\frac{\Gamma_{k, \lambda}^{L E} b_{\lambda, a}^{L E}+\left(\mu_{2}^{L E}-\Gamma_{\lambda, \lambda}^{L E}\right) b_{k, a}^{L E}}{\Gamma_{k, \lambda}^{L E} \mu_{2}^{L E}}\right] e^{-\mu_{2}^{L E} T}
$$

As a result $\lambda(0)>0$ if and only if $\Gamma_{k, \lambda}^{L E} b_{\lambda, a}^{L E}+\left(\mu_{2}^{L E}-\Gamma_{\lambda, \lambda}^{L E}\right) b_{k, a}^{L E}<0$.

Proof of Theorem 2. Given the proofs and results of lemmas 7 through 9, to prove this theorem we must establish that when $\gamma_{N}>\gamma_{N}^{*}$ and $\psi_{I}>\psi_{I}^{+}$three results hold: (1) $c(0)>0$ if and only if $\lambda(0)>0$. (2) Consumption, investment and hours will comove procyclically for all time $t<T$ if $\forall t<T, \dot{\lambda} \geq 0$ and $\dot{k} \geq 0$. (3) if $\lambda(0)>0$ then $\dot{\lambda} \geq 0$ and $\dot{k} \geq 0$.

(1): $c(0)>0$ if and only if $\lambda(0)>0$ and $\gamma_{N}>\gamma_{N}^{*}$ follows from the observation that we can substitute 22 into 23, and the result into 51 to get an equation of the form $c(0)=\zeta^{L E} \lambda(0)$ where $\zeta^{L E}=\frac{-\phi_{I}^{L E}}{\gamma_{I}^{L E}+\phi_{I}^{L E} \sigma+\psi_{I}\left(1-\phi_{I}^{L E}\right)} \cdot \zeta^{L E}>0$ follows trivially from $\psi_{I}>\psi_{I}^{+}$and $\gamma_{N}>\gamma_{N}^{*} \Rightarrow$ $\phi^{L E}<0$.

(2): We can solve for and define $x=\tau_{x, k}^{L E} k+\tau_{x, \lambda}^{L E} \lambda+\tau_{x, a}^{L E} a$ for $x=c, i, n$. Here $\tau_{c, k}^{L E}, \tau_{c, \lambda}^{L E}$, $\tau_{i, k}^{L E}, \tau_{i, \lambda}^{L E}, \tau_{n, k}^{L E}$, and $\tau_{n, \lambda}^{L E}$ are all positive ${ }^{41}$, as result it trivially follows that if $\forall t<T, \dot{\lambda} \geq 0$ and $\dot{k} \geq 0$ then consumption, investment and hours will comove procyclically for all time $t<T$.

(3): The dynamic system given by (26) takes the same form as the dynamic system given by (17). As a result showing that $\dot{\lambda} \geq 0$ and $\dot{k} \geq 0$ if $\lambda(0)>0$ amounts, exactly as in lemma 4, to proving that $\Gamma_{k, \lambda}^{L E}>0$ and $\Gamma_{\lambda, \lambda}^{L E}>0 .{ }^{42} \Gamma_{k, \lambda}^{L E}>0$ and $\Gamma_{\lambda, \lambda}^{L E}>0$ follow from $\psi_{I}>\psi_{I}^{+}$and $\gamma_{N}>\gamma_{N}^{*} \Rightarrow \phi^{L E}<0$.

\footnotetext{
${ }^{40}$ For the proof see Lemma B.10 in Appendix B (Supplementary Appendix).

${ }^{41}$ For the proof see Lemma B.9 in Appendix B (Supplementary Appendix).

${ }^{42}$ For the proof see Lemma B.10 in Appendix B (Supplementary Appendix).
} 
Results (1) - (3) together establish that if $\gamma_{N}>\gamma_{N}^{*}, \psi_{I}>\psi_{I}^{+}$, and $\lambda(0)>0$ then the labor externality model exhibits procyclical technology news shocks. From lemma 9 we further know that $\lambda(0)>0$ if and only if $\psi_{I}>\psi_{I}^{*}$.

Proof of Theorem 3. $\Gamma^{F C}=\Gamma^{P C}$ and $\tau_{x, y}^{F C}=\tau_{x, y}^{P C}$ for $x=i, c, n$ and $y=k, \lambda$. Hence, for a model with forward compatible investment lemmas 1 through 4 still hold as before.

Now, recall $\mu_{2}^{F C}=\mu_{2}^{P C}>0$. Also, $\Gamma_{k, \lambda}^{F C}=\Gamma_{k, \lambda}^{P C}>0$. For a stable solution we need:

$$
\lambda(0)=-\left[\frac{\Gamma_{k, \lambda}^{F C}\left(b_{\lambda, q}^{F C}+\tau \mu_{2}^{F C} b_{\lambda, p}^{F C}\right)+\left(\mu_{2}^{F C}-\Gamma_{\lambda, \lambda}^{F C}\right)\left(b_{k, q}^{F C}+\tau \mu_{2}^{F C} b_{k, p}^{F C}\right)}{\Gamma_{k, \lambda}^{F C} \mu_{2}^{F C}}\right] e^{-\mu_{2}^{F C} T}
$$

As a result $\lambda(0)>0$ if and only if $\tau>\frac{\Gamma_{k, \lambda}^{F C} b_{\lambda, q}^{F C}+\left(\mu_{2}^{F C}-\Gamma_{\lambda, \lambda}^{F C}\right) b_{k, q}^{F C}}{\Gamma_{k, \lambda}^{F C} \mu_{2}^{F C} b_{\lambda, p_{\epsilon}}^{F C}+\left(\mu_{2}^{F C}-\Gamma_{\lambda, \lambda}^{F C}\right) \mu_{2}^{F C} b_{k, p_{\epsilon}}^{F C}}$.

$\Leftarrow$. If $v(1-\alpha)>1$ and $\tau>\frac{\Gamma_{k, \lambda}^{F C} b_{\lambda, q}^{F C}+\left(\mu_{2}^{F C}-\Gamma_{\lambda, \lambda}^{F C}\right) b_{k, q}^{F C}}{\Gamma_{k, \lambda}^{F C} \mu_{2}^{F C} b_{\lambda, p_{\epsilon}}^{F C}+\left(\mu_{2}^{F C}-\Gamma_{\lambda, \lambda}^{F C}\right) \mu_{2}^{F C} b_{k, p_{\epsilon}}^{F C}}$, then a investment technology news shock is procyclical. Lemmas 2 and the result above prove the procyclical comovement at $t=0$, while Lemmas 3,4 and the result above establish the procyclical comovement for $0<t<T$.

$\Rightarrow$. If $v(1-\alpha)<1$ or $\tau>\frac{\Gamma_{k, \lambda}^{F C} b_{\lambda, q}^{F C}+\left(\mu_{2}^{F C}-\Gamma_{\lambda, \lambda}^{F C}\right) b_{k, q}^{F C}}{\Gamma_{k, \lambda}^{F C} \mu_{2}^{F C} b_{\lambda, p_{\epsilon}}^{F C}+\left(\mu_{2}^{F C}-\Gamma_{\lambda, \lambda}^{F C}\right) \mu_{2}^{F C} b_{k, p_{\epsilon}}^{F C}}$, then a technology news shock is not procyclical. This follow trivially from Lemma 2 , as the procyclical comovement will not occur at time $t=0$ if either of the above conditions are not met.

\section{B The Model Economies (Supplementary Appendix For Online Publication)}

\section{B.1 A Model with Production Complementarities}

\section{B.1.1 The Model Economy}

A social planner has the following preferences

$$
U=(1-\sigma)^{-1} \int_{0}^{\infty} e^{-\rho t}[C(t) \exp (-N(t))]^{1-\sigma} d t
$$

over time paths for consumption $C$ and hours worked $N$. We assume this functional form for the utility to preserve balanced growth. Also, $\rho=1 / \beta-1>0$ and $\sigma \geq 0$, where $\beta$ is the 
subjective discount factor and $\sigma$ is the inverse of the intertemporal elasticity of substitution.

The planner is subject to the following constraints:

$$
\begin{aligned}
F[C(t), I(t)] & =K(t)^{\alpha}(A(t) N(t))^{1-\alpha} \\
\dot{K}(t) & =I(t)-\delta K(t)
\end{aligned}
$$

Here $K, I$ and $A$ represent capital, investment and the level of technology. The path of technology and the initial capital stock are exogenous. The depreciation rate, $\delta$, and the elasticity of output with respect to capital, $\alpha$, both lie between zero and one.

Further, we assume:

$$
F(C, I) \equiv\left[\theta C^{v}+(1-\theta) I^{v}\right]^{1 / v}
$$

where $\theta \in(0,1)$ and $v \geq 1$. When $v=1$, the equation collapses to the standard neo-classical case, which has infinite substitutability between the two goods. As $v$ increases, the complementarity between the production of the two goods increases. If $v=\infty$, the production frontier takes a Leontief form.

Next, let us define the exogenous processes - the technology news shock. The planner again has perfect foresight, with

$$
A(t)=\left\{\begin{array}{cc}
\bar{A} & \text { for } t \in[0, T) \\
\tilde{A}=1.01 \times \bar{A} & t \geq T
\end{array}\right.
$$

For the contemporaneous improvements case $T=0$ in the above specification.

\section{B.1.2 The Model Economy's First Order Conditions}

The social planner chooses $C, I, K$, and $N$ to maximize $U$ subject to (54) and (55) taking as

given the initial condition $K(0)$ and time path of technology. We can express the problem as a current value Hamiltonian:

$$
H=C^{1-\sigma} \exp [-(1-\sigma) N]+\Lambda(I-\delta K)+\Phi\left(K^{\alpha}(A N)^{1-\alpha}-F(C, I)\right)
$$


The first-order necessary conditions at an interior solution satisfy :

$$
\begin{gathered}
-\frac{U_{N}}{U_{C}}=(1-\alpha) \frac{F}{N}\left(F_{C}\right)^{-1} \\
\frac{U_{C}}{\Lambda}=\frac{F_{C}}{F_{I}} \\
\frac{\dot{\Lambda}}{\Lambda}-\rho=\delta-\alpha \frac{F}{K}\left(F_{I}\right)^{-1}
\end{gathered}
$$

along with our initial condition on capital and a transversality condition on $\Lambda$.

Equation (56) is the intratemporal Euler equation between consumption and labor hours, equation (57) is the intratemporal Euler equation between consumption and investment, and equation (58) is the optimal capital accumulation equation.

\section{B.1.3 The Model Economy Log Linearized and Simplified}

Given the first order conditions in the previous section our model economy can be described by the following five log linearized equations:

$$
\begin{gathered}
\left(1-s_{I}\right) c+s_{I} i=\alpha k+(1-\alpha)(a+n) \\
v s_{I}(i-c)=n \\
\lambda=(1-v)(c-i)-\sigma c-\frac{(1-\sigma)(1-\alpha)}{\left(1-s_{I}\right)} n \\
\dot{k}=\delta(i-k) \\
\dot{\lambda}=-(\rho+\delta)\left[v\left(1-s_{I}\right)(c-i)+i-k\right]
\end{gathered}
$$


Here, $s_{I}=\frac{\alpha \delta}{\rho+\delta}$.

We can substitute (60) into (59) to get the consumption-investment production frontier $\left(L_{1}\right.$ line):

$$
\left(1-\phi_{I}^{P C}\right) c+\phi_{I}^{P C} i=\alpha k+(1-\alpha) a
$$

Here, $\phi_{I}^{P C}=(1-(1-\alpha) v) s_{I}$

We can also substitute (60) into (61) to get the consumption-investment euler equation $\left(L_{2}\right.$ line):

$$
\gamma_{I}^{P C} i-\left(\sigma+\gamma_{I}^{P C}\right) c=\lambda
$$

Here, $\gamma_{I}^{P C}=(v-1)-\frac{v(1-\alpha)(1-\sigma) s_{I}}{\left(1-s_{I}\right)}$.

Equations (64) and (65) now give us a system of equations in $i$ and $c$ (treating $\lambda, k$, and $a$ as exogenous).

We solve the system of equations above for $c, i, n, \dot{k}$, and $\dot{\lambda}$, assuming as given the state variable $\lambda$ and $k$, and the exogenous variable $a$ :

$$
\begin{gathered}
c=\tau_{c, k}^{P C} k+\tau_{c, \lambda}^{P C} \lambda+\tau_{c, a}^{P C} a \\
i=\tau_{i, k}^{P C} k+\tau_{i, \lambda}^{P C} \lambda+\tau_{i, a}^{P C} a \\
n=\tau_{n, k}^{P C} k+\tau_{n, \lambda}^{P C} \lambda+\tau_{n, a}^{P C} a \\
\dot{k}=\Gamma_{k, k}^{P C} k+\Gamma_{k, \lambda}^{P C} \lambda+b_{k, a}^{P C} a
\end{gathered}
$$




$$
\dot{\lambda}=\Gamma_{\lambda, k}^{P C} k+\Gamma_{\lambda, \lambda}^{P C} \lambda+b_{\lambda, a}^{P C} a
$$

where,

$$
\begin{array}{ll}
\tau_{c, k}^{P C}=\frac{\partial c}{\partial k}=\frac{\gamma_{I}^{P C} \alpha}{\phi_{I}^{P C} \sigma+\gamma_{I}^{P C}} & \Gamma_{k, k}^{P C}=\frac{\partial \dot{k}}{\partial k}=\frac{-\delta\left((1-\alpha) \gamma_{I}^{P C}+\phi_{I}^{P C} \sigma-\alpha \sigma\right)}{\phi_{I}^{P C} \sigma+\gamma_{I}^{P C}} \\
\tau_{c, \lambda}^{P C}=\frac{\partial c}{\partial \lambda}=\frac{-\phi_{I}^{P C}}{\phi_{I}^{P C} \sigma+\gamma_{I}^{P C}} & \Gamma_{k, \lambda}^{P C}=\frac{\partial \dot{k}}{\partial \lambda}=\frac{\delta\left(1-\phi_{I}^{P C}\right)}{\phi_{I}^{P C} \sigma+\gamma_{I}^{P C}} \\
\tau_{c, a}^{P C}=\frac{\partial c}{\partial a}=\frac{\gamma_{I}^{P C}(1-\alpha)}{\phi_{I}^{P C} \sigma+\gamma_{I}^{P C}} & \Gamma_{\lambda, k}^{P C}=\frac{\partial \dot{\lambda}}{\partial k}=\frac{(\rho+\delta)\left((1-\alpha) \gamma_{I}^{P C}+\phi_{I}^{P C} \sigma-\alpha \sigma+\alpha \sigma v\left(1-s_{I}\right)\right)}{\phi_{I}^{P C} \sigma+\gamma_{I}^{P C}} \\
\tau_{i, k}^{P C}=\frac{\partial i}{\partial k}=\frac{\alpha\left(\gamma_{I}^{P C}+\sigma\right)}{\phi_{I}^{P C} \sigma+\gamma_{I}^{P C}} & \Gamma_{\lambda, \lambda}^{P C}=\frac{\partial \dot{\lambda}}{\partial \lambda}=\frac{(\rho+\delta)\left(\phi_{I}^{P C}-\left(1-v\left(1-s_{I}\right)\right)\right)}{\phi_{I}^{P C} \sigma+\gamma_{I}^{P C}} \\
\tau_{i, \lambda}^{P C}=\frac{\partial i}{\partial \lambda}=\frac{1-\phi_{I}^{P C}}{\phi_{I}^{P C} \sigma+\gamma_{I}^{P C}} & b_{k, a}^{P C}=\frac{\partial \dot{k}}{\partial a}=\frac{\delta\left(\gamma_{I}^{P C}+\sigma\right)(1-\alpha)}{\phi_{I}^{P C} \sigma+\gamma_{I}^{P C}} \\
\tau_{i, a}^{P C}=\frac{\partial i}{\partial a}=\frac{(1-\alpha)\left(\gamma_{I}^{P C}+\sigma\right)}{\phi_{I}^{P C} \sigma+\gamma_{I}^{P C}} & b_{\lambda, a}^{P C}=\frac{\partial \dot{\lambda}}{\partial a}=\frac{(\rho+\delta)(1-\alpha)\left(\sigma\left(v\left(1-s_{I}\right)-1\right)-\gamma_{I}^{P C}\right)}{\phi_{I}^{P C} \sigma+\gamma_{I}^{P C}} \\
\tau_{n, k}^{P C}=\frac{\partial n}{\partial k}=\frac{v s_{I} \alpha \sigma}{\phi_{I}^{P C} \sigma+\gamma_{I}^{P C}} & \\
\tau_{n, \lambda}^{P C}=\frac{\partial n}{\partial \lambda}=\frac{v s_{I}}{\phi_{I}^{P C} \sigma+\gamma_{I}^{P C}} & \\
\tau_{n, a}^{P C}=\frac{\partial n}{\partial a}=\frac{v s_{I}(1-\alpha) \sigma}{\phi_{I}^{P C} \sigma+\gamma_{I}^{P C}} &
\end{array}
$$

Recall: $s_{I}=\frac{\alpha \delta}{\rho+\delta}, \phi_{I}^{P C}=(1-(1-\alpha) v) s_{I}$, and $\gamma_{I}^{P C}=(v-1)-\frac{v(1-\alpha)(1-\sigma) s_{I}}{\left(1-s_{I}\right)}$

\section{B.1.4 The Dynamic System}

Let us now solve the dynamic system:

$$
\left[\begin{array}{l}
\dot{\lambda}(t) \\
\dot{k}(t)
\end{array}\right]=\left[\begin{array}{cc}
\Gamma_{\lambda, \lambda}^{P C} & \Gamma_{\lambda, k}^{P C} \\
\Gamma_{k, \lambda}^{P C} & \Gamma_{k, k}^{P C}
\end{array}\right]\left[\begin{array}{c}
\lambda(t) \\
k(t)
\end{array}\right]+\left[\begin{array}{c}
b_{\lambda, a}^{P C} \\
b_{k, a}^{P C}
\end{array}\right] a(t)
$$

In order to solve this system we must first determine the eigenvalues of the $\Gamma$ matrix. For now we assume that a stable solution exists and that one of the eigenvalues is positive and 
the other negative. We will later prove this to be true. Let us label the eigenvalues $\mu_{1}^{P C}$ and $\mu_{2}^{P C}$ and without loss of generality, we will assume henceforth that $\mu_{1}^{P C}<0$ and $\mu_{2}^{P C}>0$.

We now introduce the technology news shock - a permanent increase in technology in period T. Specifically,

$$
a(t)=w(t)=\left\{\begin{array}{cc}
0 & \text { for } t \in[0, T) \\
1 & t \geq T
\end{array}\right.
$$

To analyze the resulting system, it will be useful to introduce the Laplace transform operator.

The Laplace transform of a function $p(t)$ is:

$$
\mathcal{L}[p(t)]=\bar{P}(s)=\int_{0}^{\infty} e^{-s t} p(t) d t
$$

We will use $\bar{P}$ rather than $P$ to distinguish the Laplace transform of the log deviation of a variables from the level of said variable.

Moreover, we know from Theorem 6.3 from Boyce and Diprima (1969), that

$$
\mathcal{L}\left[p^{\prime}(t)\right]=s \mathcal{L}(p(t))-p(0)
$$

Taking the Laplace transform of the differential equations in $\left[\begin{array}{ll}\lambda & k\end{array}\right]^{\prime}$ and applying this theorem, we get:

$$
\left[\begin{array}{l}
\bar{\Lambda}(s) \\
\bar{K}(s)
\end{array}\right]=(s I-\Gamma)^{-1}\left\{\left[\begin{array}{l}
\lambda(0) \\
k(0)
\end{array}\right]+\left[\begin{array}{c}
b_{\lambda, a}^{P C} \\
b_{k, a}^{P C}
\end{array}\right] \bar{W}(s)\right\}
$$

Given (70), it can be shown that

$$
\bar{W}(s)=\mathcal{L}[w(t)]=\frac{1}{s} e^{-s T}
$$


Rewriting equation (71), we get:

$$
\left[\begin{array}{l}
\bar{\Lambda}(s) \\
\bar{K}(s)
\end{array}\right]=\frac{1}{\left(s-\mu_{1}^{P C}\right)\left(s-\mu_{2}^{P C}\right)}\left[\begin{array}{cc}
s-\Gamma_{k, k}^{P C} & \Gamma_{\lambda, k}^{P C} \\
\Gamma_{k, \lambda}^{P C} & s-\Gamma_{\lambda, \lambda}^{P C}
\end{array}\right]\left\{\left[\begin{array}{c}
\lambda(0) \\
k(0)
\end{array}\right]+\left[\begin{array}{c}
b_{\lambda, a}^{P C} \\
b_{k, a}^{P C}
\end{array}\right] \bar{W}(s)\right\}
$$

Remember, $\mu_{1}^{P C}$ and $\mu_{2}^{P C}$ are the eigenvalues of $\Gamma^{P C}$, and $\mu_{1}^{P C}<0$ and $\mu_{2}^{P C}>0$.

The lower row of (72) gives us:

$$
\bar{K}(s)=\frac{\Gamma_{k, \lambda}^{P C} \lambda(0)+\left(s-\Gamma_{\lambda, \lambda}^{P C}\right) k(0)}{\left(s-\mu_{1}^{P C}\right)\left(s-\mu_{2}^{P C}\right)}+\left[\frac{\Gamma_{k, \lambda}^{P C} b_{\lambda, a}^{P C}+\left(s-\Gamma_{\lambda, \lambda}^{P C}\right) b_{k, a}^{P C}}{s\left(s-\mu_{1}^{P C}\right)\left(s-\mu_{2}^{P C}\right)}\right] e^{-s T}
$$

Next, we take the inverse Laplace transform of $K(s)$ to recover $k$ as a function of time. After some algebra,

$$
\begin{aligned}
k(t)= & \frac{\Gamma_{k, \lambda}^{P C} \lambda(0)}{\mu_{1-}^{P C} \mu_{2}^{P C}} e^{\mu_{1}^{P C} t}+\frac{\Gamma_{k, \lambda}^{P C} \lambda(0)}{\mu_{2-}^{P C} \mu_{1}^{P C}} e^{\mu_{2}^{P C} t}+\frac{\left(\mu_{1}^{P C}-\Gamma_{\lambda, \lambda}^{P C}\right) k(0)}{\mu_{1}^{P C}-\mu_{2}^{P C}} e^{\mu_{1}^{P C} t}+\frac{\left(\mu_{2}^{P C}-\Gamma_{\lambda, \lambda}^{P C}\right) k(0)}{\mu_{2}^{P C}-\mu_{1}^{P C}} e^{\mu_{2}^{P C} t} \\
& +u_{T}(t)\left(\frac{\Gamma_{k, \lambda}^{P C} b_{\lambda, a}^{P C}-\Gamma_{\lambda, \lambda}^{P C} b_{k, a}^{P C}}{\mu_{1}^{P C} \mu_{2}^{P C}}\right) \\
& +u_{T}(t)\left(\frac{\Gamma_{k, \lambda}^{P C} b_{\lambda, a}^{P C}+\left(\mu_{1}^{P C}-\Gamma_{\lambda, \lambda}^{P C}\right) b_{k, a}^{P C}}{\mu_{1}^{P C}\left(\mu_{1}^{P C}-\mu_{2}^{P C}\right)} e^{\mu_{1}^{P C}(t-T)}\right) \\
& +u_{T}(t)\left(\frac{\Gamma_{k, \lambda}^{P C} b_{\lambda, a}^{P C}+\left(\mu_{2}^{P C}-\Gamma_{\lambda, \lambda}^{P C}\right) b_{k, a}^{P C}}{\mu_{2}^{P C}\left(\mu_{2}^{P C}-\mu_{1}^{P C}\right)} e^{\mu_{2}^{P C}(t-T)}\right)
\end{aligned}
$$

where $u_{T}(t)$ is a step function that takes on a value of one for all $t \geq T$, and zero otherwise.

Recall that we assume the initial capital stock is at the steady-state level associated with the pre-shock technology level. As such, $k(0)=0$ : 


$$
\begin{aligned}
k(t)= & \frac{\Gamma_{k, \lambda}^{P C} \lambda(0)}{\mu_{1-}^{P C} \mu_{2}^{P C}} e^{\mu_{1}^{P C} t}+\frac{\Gamma_{k, \lambda}^{P C} \lambda(0)}{\mu_{2-}^{P C} \mu_{1}^{P C}} e^{\mu_{2}^{P C} t} \\
& +u_{T}(t)\left(\frac{\Gamma_{k, \lambda}^{P C} b_{\lambda, a}^{P C}-\Gamma_{\lambda, \lambda}^{P C} b_{k, a}^{P C}}{\mu_{1}^{P C} \mu_{2}^{P C}}\right) \\
& +u_{T}(t)\left(\frac{\Gamma_{k, \lambda}^{P C} b_{\lambda, a}^{P C}+\left(\mu_{1}^{P C}-\Gamma_{\lambda, \lambda}^{P C}\right) b_{k, a}^{P C}}{\mu_{1}^{P C}\left(\mu_{1}^{P C}-\mu_{2}^{P C}\right)} e^{\mu_{1}^{P C}(t-T)}\right) \\
& +u_{T}(t)\left(\frac{\Gamma_{k, \lambda}^{P C} b_{\lambda, a}^{P C}+\left(\mu_{2}^{P C}-\Gamma_{\lambda, \lambda}^{P C}\right) b_{k, a}^{P C}}{\mu_{2}^{P C}\left(\mu_{2}^{P C}-\mu_{1}^{P C}\right)} e^{\mu_{2}^{P C}(t-T)}\right)
\end{aligned}
$$

This gives us the solution to a differential equation with one undetermined variable $\lambda(0)$. We now seek a path for $\left[\begin{array}{ll}\lambda & k\end{array}\right]^{\prime}$ that is not explosive. In order to achieve this, we choose $\lambda(0)$ such that the explosive root $\mu_{2}^{P C}$ is 'zeroed out' for all $t>T$. Otherwise, the path for $k(t)$ will be explosive. This restriction on $\lambda(0)$ is:

$$
\left(\frac{\Gamma_{k, \lambda}^{P C}}{\mu_{2}^{P C}-\mu_{1}^{P C}}\right) \lambda(0)=-\frac{\Gamma_{k, \lambda}^{P C} b_{\lambda, a}^{P C}+\left(\mu_{2}^{P C}-\Gamma_{\lambda, \lambda}^{P C}\right) b_{k, a}^{P C}}{\mu_{2}^{P C}\left(\mu_{2}^{P C}-\mu_{1}^{P C}\right)} e^{-\mu_{2}^{P C} T}
$$

This can be re-written as:

$$
\lambda(0)=-\left[\frac{\Gamma_{k, \lambda}^{P C} b_{\lambda, a}^{P C}+\left(\mu_{2}^{P C}-\Gamma_{\lambda, \lambda}^{P C}\right) b_{k, a}^{P C}}{\Gamma_{k, \lambda}^{P C} \mu_{2}^{P C}}\right] e^{-\mu_{2}^{P C} T}
$$

Let us also solve the second half of our laplace transform. This will allow us to study the path of $\lambda(t)$ over time. The first row of (72) gives us:

$$
\bar{\Lambda}(s)=\frac{\left(s-\Gamma_{k, k}^{P C}\right) \lambda(0)+\Gamma_{\lambda, k}^{P C} k(0)}{\left(s-\mu_{1}^{P C}\right)\left(s-\mu_{2}^{P C}\right)}+\left[\frac{\left(s-\Gamma_{k, k}^{P C}\right) b_{\lambda, a}^{P C}+\Gamma_{\lambda, k}^{P C} b_{k, a}^{P C}}{s\left(s-\mu_{1}^{P C}\right)\left(s-\mu_{2}^{P C}\right)}\right] e^{-s T}
$$

Now we can take the inverse Laplace transform of $\Lambda(s)$ to recover $\lambda$ as a function of time. After some algebra and setting $k(0)=0$ we get: 


$$
\begin{aligned}
\lambda(t)= & \frac{\left(\mu_{1}^{P C}-\Gamma_{k, k}^{P C}\right) \lambda(0)}{\mu_{1-}^{P C} \mu_{2}^{P C}} e^{\mu_{1}^{P C} t}+\frac{\left(\mu_{2}^{P C}-\Gamma_{k, k}^{P C}\right) \lambda(0)}{\mu_{2-}^{P C} \mu_{1}^{P C}} e^{\mu_{2}^{P C} t} \\
& +u_{T}(t)\left(\frac{\Gamma_{\lambda, k}^{P C} b_{k, a}^{P C}-\Gamma_{k, k}^{P C} b_{\lambda, a}^{P C}}{\mu_{1}^{P C} \mu_{2}^{P C}}\right) \\
& +u_{T}(t)\left(\frac{\left(\mu_{1}^{P C}-\Gamma_{k, k}^{P C}\right) b_{\lambda, a}^{P C}+\Gamma_{\lambda, k}^{P C} b_{k, a}^{P C}}{\mu_{1}^{P C}\left(\mu_{1}^{P C}-\mu_{2}^{P C}\right)} e^{\mu_{1}^{P C}(t-T)}\right) \\
& +u_{T}(t)\left(\frac{\left(\mu_{2}^{P C}-\Gamma_{k, k}^{P C}\right) b_{\lambda, a}^{P C}+\Gamma_{\lambda, k}^{P C} b_{k, a}^{P C}}{\mu_{2}^{P C}\left(\mu_{2}^{P C}-\mu_{1}^{P C}\right)} e^{\mu_{2}^{P C}(t-T)}\right)
\end{aligned}
$$

Given that we choose a $\lambda(0)$ such that the explosive root $\mu_{2}^{P C}$ is 'zeroed out' for all $t>T$, we can simplify our equations for the time paths of $k(t)$ and $\lambda(t)$ to the following:

$$
k(t)=\left\{\begin{array}{cc}
\frac{\Gamma_{k, \lambda}^{P C} \lambda(0)}{\mu_{1}^{P C}-\mu_{2}^{P C}} e^{\mu_{1}^{P C} t}+\frac{\Gamma_{, \lambda}^{P C} \lambda(0)}{\mu_{2}^{P C}-\mu_{1}^{P C}} e^{\mu_{2}^{P C} t} & \text { for } t \in[0, T) \\
\frac{\Gamma_{k, \lambda}^{P C} \lambda(0)}{\mu_{1-}^{P C} \mu_{2}^{P C}} e^{\mu_{1}^{P C} t}+\frac{\Gamma_{k, \lambda}^{P C} b b_{\lambda, a}^{P C}-\Gamma_{\lambda, \lambda}^{P C} b_{k, a}^{P C}}{\mu_{1}^{P P C} \mu_{2}^{P C}}+\frac{\Gamma_{k, \lambda}^{P C} b_{\lambda, a}^{P C}+\left(\mu_{1}^{P C}-\Gamma_{\lambda, \lambda}^{P C}\right) b_{k, a}^{P C}}{\mu_{1}^{P C}\left(\mu_{1}^{P C}-\mu_{2}^{P C}\right)} e^{\mu_{1}^{P C}(t-T)} & t \geq T
\end{array}\right.
$$

$\lambda(t)=\left\{\begin{array}{cc}\frac{\left(\mu_{1}^{P C}-\Gamma_{k, k}^{P C}\right) \lambda(0)}{\mu_{1}^{P C}-\mu_{2}^{P C}} e^{\mu_{1}^{P C} t}+\frac{\left(\mu_{2}^{P C}-\Gamma_{k, k}^{P C}\right) \lambda(0)}{\mu_{2}^{P C} \mu_{1}^{P C}} e^{\mu_{2}^{P C} t} & \text { for } t \in[0, T) \\ \frac{\left(\mu_{1}^{P C}-\Gamma_{k, k}^{P C}\right) \lambda(0)}{\mu_{1}^{P C}-\mu_{2}^{P C}} e^{\mu_{1}^{P C} t}+\frac{\Gamma_{\lambda, k}^{P C} b_{k, a}^{P C}-\Gamma_{k, k}^{P C} b_{\lambda, a}^{P C}}{\mu_{1}^{P C} \mu_{2}^{P C}}+\frac{\Gamma_{\lambda, k}^{P C} b_{k, a}^{P C}+\left(\mu_{1}^{P C}-\Gamma_{k, k}^{P C}\right) b_{\lambda, a}^{P C}}{\mu_{1}^{P C}\left(\mu_{1}^{P C}-\mu_{2}^{P C}\right)} e^{\mu_{1}^{P C}(t-T)} & t \geq T\end{array}\right.$

Equations (66), (67), (68), (74), and (75), along with equation (73) give us a stable solution to our model economy for a $1 \%$ technology shock that occurs in period $T$.

\section{B.1.5 Proofs \& Expressions}

In this section we will sign the various expressions needed for Lemma 1-5 and Theorem 1. 
First, let us recall the proof for Lemma 1. For consumption, investment and hours to comove at time zero (on impact of the news) we required $v(1-\alpha)>1$ which resulted in $\phi_{I}^{P C}<0$ and thus a positively sloped $L_{1}$ line. For this section we will assume that $v>(1-\alpha)^{-1}$

Assumption: $v>v^{*}=(1-\alpha)^{-1}$

Lemma B.1: $\gamma_{I}^{P C}>0$

Proof.

$$
\begin{aligned}
\gamma_{I}^{P C} & =(v-1)-\frac{v(1-\alpha)(1-\sigma) s_{I}}{\left(1-s_{I}\right)} \\
& =(v-1)-\frac{(1-\alpha) \delta}{(\rho+(1-\alpha) \delta)}(1-\sigma) v \alpha \\
& >(v-1)-v \alpha \\
& =v(1-\alpha)-1>0
\end{aligned}
$$

Lemma B.2: The slope of the $L_{2}$ line in the consumption-investment space is positive.

Proof. The $L_{2}$ line is given by:

$$
i=\frac{\left(\sigma+\gamma_{I}^{P C}\right)}{\gamma_{I}^{P C}} c+\frac{1}{\gamma_{I}^{P C}} \lambda
$$

If $\gamma_{I}^{P C}>0$ then the slope, $\frac{\left(\sigma+\gamma_{I}^{P C}\right)}{\gamma_{I}^{P C}}$, must be positive.

Lemma B.3: $\phi_{I}^{P C} \sigma+\gamma_{I}^{P C}>0$ 
Proof.

$$
\begin{aligned}
\phi_{I}^{P C} \sigma+\gamma_{I}^{P C} & =(1-(1-\alpha) v) s_{I} \sigma+(v-1)-\frac{v(1-\alpha)(1-\sigma) s_{I}}{\left(1-s_{I}\right)} \\
& =s_{I} \sigma-(1-\alpha) v s_{I} \sigma+(v-1)-\frac{\left((1-\alpha) v s_{I}-(1-\alpha) v s_{I} \sigma\right)}{1-s_{I}} \\
& =s_{I} \sigma+(v-1)-\frac{(1-\alpha) v s_{I}}{1-s_{I}}+\frac{(1-\alpha) v s_{I}^{2} \sigma}{1-s_{I}} \\
& =v-\left(1-\sigma s_{I}\right)\left[1+(1-\alpha) v \frac{s_{I}}{1-s_{I}}\right] \\
& =v\left\{1-\left(1-\sigma s_{I}\right)\left[\frac{1}{v}+(1-\alpha) \frac{s_{I}}{1-s_{I}}\right]\right\}>0
\end{aligned}
$$

The last inequality follows from the following observations:

If $\left(1-\sigma s_{I}\right)<0$, then we are done. If $\left(1-\sigma s_{I}\right)>0$, then we can define:

$$
\chi(v)=\left\{1-\left(1-\sigma s_{I}\right)\left[\frac{1}{v}+(1-\alpha) \frac{s_{I}}{1-s_{I}}\right]\right\}
$$

Now,

$$
\begin{aligned}
\chi(\infty) & =\left\{1-\left(1-\sigma s_{I}\right)(1-\alpha) \frac{s_{I}}{1-s_{I}}\right\} \\
& =\left\{1-\left(1-\sigma s_{I}\right) \alpha \frac{(1-\alpha) \delta}{\rho+(1-\alpha) \delta}\right\}>0
\end{aligned}
$$

$$
\begin{aligned}
\chi\left(v_{c}\right) & =\left\{1-\left(1-\sigma s_{I}\right)\left[(1-\alpha)+(1-\alpha) \frac{s_{I}}{1-s_{I}}\right]\right\} \\
& =\left[1-\left(1-\sigma s_{I}\right) \frac{(1-\alpha)}{1-s_{I}}\right] \\
& =\left[1-\left(1-\sigma s_{I}\right) \frac{(1-\alpha) \rho+(1-\alpha) \delta}{\rho+(1-\alpha) \delta}\right]>0
\end{aligned}
$$




$$
\chi^{\prime}(v)=\frac{\left(1-\sigma s_{I}\right)}{v^{2}}>0
$$

Therefore, for $v \in\left(v_{c}, \infty\right), \chi(v)>0$. Given Assumption 1 this translates to $\chi(v)>0$

Lemma B.4: $\tau_{c, k}^{P C}, \tau_{c, \lambda}^{P C}, \tau_{c, a}^{P C}, \tau_{i, k}^{P C}, \tau_{i, \lambda}^{P C}, \tau_{i, a}^{P C}, \tau_{n, k}^{P C}, \tau_{n, \lambda}^{P C}$, and $\tau_{n, a}^{P C}$ are all positive

Proof. This result follows trivially lemma's B.2 and B.3 and our assumption, $v>(1-\alpha)^{-1}$, which ensures $\phi_{I}^{P C}<0$.

Lemma B.5: $\Gamma_{k, \lambda}^{P C}$ and $\Gamma_{\lambda, \lambda}^{P C}$ are both positive.

Proof. $\Gamma_{k, \lambda}^{P C}>0$ follows trivially from lemma B.3 and our assumption, $v>(1-\alpha)^{-1} \Rightarrow$ $\phi_{I}^{P C}<0$.

To prove $\Gamma_{\lambda, \lambda}^{P C}=\frac{(\rho+\delta)\left(\phi_{I}^{P C}-\left(1-v\left(1-s_{I}\right)\right)\right)}{\phi_{I}^{P C} \sigma+\gamma_{I}^{P C}}>0$ it suffices to show $\left(\phi_{I}^{P C}-\left(1-v\left(1-s_{I}\right)\right)\right)>0$, because by lemma B.3 we know $\phi_{I}^{P C} \sigma+\gamma_{I}^{P C}>0$.

$$
\begin{aligned}
\phi_{I}^{P C}-\left(1-v\left(1-s_{I}\right)\right) & =\frac{(1-v(1-\alpha)) \alpha \delta}{\rho+\delta}+\frac{(v-1) \rho+(v(1-\alpha)-1) \delta}{\rho+\delta} \\
& =\frac{(v-1) \rho+(1-\alpha)(v(1-\alpha)-1) \delta}{\rho+\delta}>0
\end{aligned}
$$

Lemma B.4 above proves that $\Gamma_{k, \lambda}^{P C}$ and $\Gamma_{\lambda, \lambda}^{P C}$ are both positive. For our analysis we do not need to sign $\Gamma_{k, k}^{P C}$ and $\Gamma_{\lambda, k}^{P C} 43$.

Lemma B.6: One of the eigenvalues of the $\Gamma^{P C}$ matrix is positive and other negative.

\footnotetext{
${ }^{43}$ It can be shown that both these variables are positive for $\sigma \in[0,1]$
} 
Proof. The product of the eigenvalues is given by the determinant of $\Gamma^{P C}$ :

$$
\begin{aligned}
\operatorname{det}\left(\Gamma^{P C}\right)= & \frac{-\delta(\rho+\delta)}{\left(\phi_{I}^{P C} \sigma+\gamma_{I}^{P C}\right)^{2}}\left[\left(\alpha\left(\gamma_{I}^{P C}+\sigma\right)-\phi_{I}^{P C} \sigma-\gamma_{I}^{P C}\right)\left(\left(1-v\left(1-s_{I}\right)\right)-\phi_{I}^{P C}\right)\right] \\
& +\frac{-\delta(\rho+\delta)}{\left(\phi_{I}^{P C} \sigma+\gamma_{I}^{P C}\right)^{2}}\left[\left(1-\phi_{I}^{P C}\right)\left((1-\alpha) \gamma_{I}^{P C}-\left(\alpha-\phi_{I}^{P C}\right) \sigma+\alpha v\left(1-s_{I}\right) \sigma\right)\right] \\
= & \frac{-\delta(\rho+\delta)}{\left(\phi_{I}^{P C} \sigma+\gamma_{I}^{P C}\right)^{2}}\left[\left((\alpha-1) \gamma_{I}^{P C}-\left(\phi_{I}^{P C}-\alpha\right) \sigma\right)\left(\left(1-v\left(1-s_{I}\right)\right)-\phi_{I}^{P C}\right)\right] \\
& +\frac{-\delta(\rho+\delta)}{\left(\phi_{I}^{P C} \sigma+\gamma_{I}^{P C}\right)^{2}}\left[\left(1-\phi_{I}^{P C}\right)\left((1-\alpha) \gamma_{I}^{P C}-\left(\alpha-\phi_{I}^{P C}\right) \sigma+\alpha v\left(1-s_{I}\right) \sigma\right)\right] \\
= & \frac{-\delta(\rho+\delta)}{\left(\phi_{I}^{P C} \sigma+\gamma_{I}^{P C}\right)^{2}}\left[\left((1-\alpha) \gamma_{I}^{P C}-\left(\alpha-\phi_{I}^{P C}\right) \sigma\right) v\left(1-s_{I}\right)-\left(1-\phi_{I}^{P C}\right) \alpha \sigma v\left(1-s_{I}\right)\right] \\
= & \frac{-\delta(\rho+\delta)}{\left(\phi_{I}^{P C} \sigma+\gamma_{I}^{P C}\right)^{2}}\left[\left((1-\alpha) \gamma_{I}^{P C}-\left(\alpha-\phi_{I}^{P C}\right) \sigma\right) v\left(1-s_{I}\right)-\left(1-\phi_{I}^{P C}\right) \alpha \sigma v\left(1-s_{I}\right)\right] \\
= & \frac{-\delta(\rho+\delta)}{\left(\phi_{I}^{P C} \sigma+\gamma_{I}^{P C}\right)^{2}}\left[v\left(1-s_{I}\right)(1-\alpha)\left(\gamma_{I}^{P C}+\sigma \phi_{I}^{P C}\right)\right] \\
\quad-\delta(\rho+\delta) & -\left(\phi_{I}^{P C} \sigma+\gamma_{I}^{P C}\right)\left[v\left(1-s_{I}\right)(1-\alpha)\right]<0
\end{aligned}
$$

As the product of the eigenvalues is negative it follows that the eigenvalues have opposite signs.

Lemma B.7: The sum of the eigenvalues of the $\Gamma^{P C}$ matrix is $\rho$.

Proof. The sum of the eigenvalues is given by the trace of $\Gamma^{P C}$ :

$$
\begin{aligned}
\operatorname{tr}\left(\Gamma^{P C}\right) & =\Gamma_{\lambda, \lambda}^{P C}+\Gamma_{k, k}^{P C} \\
& =\frac{\delta\left((\alpha-1) \gamma_{I}^{P C}+\alpha \sigma-\phi_{I}^{P C} \sigma\right)-\delta\left(1-v\left(1-s_{I}\right)-\phi_{I}^{P C}\right)-\rho\left(1-v\left(1-s_{I}\right)-\phi_{I}^{P C}\right)}{\phi_{I}^{P C} \sigma+\gamma_{I}^{P C}} \\
& =\frac{-\delta(1-\alpha) \gamma_{I}^{P C}+\sigma \delta\left(\alpha-\phi_{I}^{P C}\right)+(\rho+\delta) \phi_{I}^{P C}-(\rho+\delta)\left(1-v\left(1-s_{I}\right)\right)}{\phi_{I}^{P C} \sigma+\gamma_{I}^{P C}}
\end{aligned}
$$




$$
\begin{aligned}
& =\frac{-\delta(1-\alpha) \gamma_{I}^{P C}+\sigma \delta\left(\alpha-\phi_{I}^{P C}\right)+(1-(1-\alpha) v) \alpha \delta+(v-1) \rho+(v(1-\alpha)-1) \delta}{\phi_{I}^{P C} \sigma+\gamma_{I}^{P C}} \\
& =\frac{-\delta(1-\alpha) \gamma_{I}^{P C}+\sigma \delta\left(\alpha-\phi_{I}^{P C}\right)+(v-1) \rho+(1-\alpha)(v(1-\alpha)-1) \delta}{\phi_{I}^{P C} \sigma+\gamma_{I}^{P C}} \\
& =\frac{-\delta(1-\alpha) \gamma_{I}^{P C}+\sigma \delta\left(\alpha-\phi_{I}^{P C}\right)+\gamma_{I}^{P C}(\rho+(1-\alpha) \delta)-\sigma(v(1-\alpha) \alpha \delta)}{\phi_{I}^{P C} \sigma+\gamma_{I}^{P C}} \\
& =\frac{\rho \gamma_{I}^{P C}+\sigma \delta\left(\alpha-\phi_{I}^{P C}-v \alpha(1-\alpha)\right)}{\phi_{I}^{P C} \sigma+\gamma_{I}^{P C}} \\
& =\frac{\rho \gamma_{I}^{P C}+\sigma \delta\left(\alpha(1-v(1-\alpha))-\phi_{I}^{P C}\right)}{\phi_{I}^{P C} \sigma+\gamma_{I}^{P C}} \\
& =\frac{\rho \gamma_{I}^{P C}+\sigma \delta\left(\left(\alpha-s_{I}\right)(1-v(1-\alpha))\right)}{\phi_{I}^{P C} \sigma+\gamma_{I}^{P C}} \\
& =\frac{\rho\left(\gamma_{I}^{P C}+\sigma s_{I}(1-v(1-\alpha))\right)}{\phi_{I}^{P C} \sigma+\gamma_{I}^{P C}} \\
& =\frac{\rho\left(\phi_{I}^{P C} \sigma+\gamma_{I}^{P C}\right)}{\phi_{I}^{P C} \sigma+\gamma_{I}^{P C}} \\
& =\rho>0
\end{aligned}
$$

\section{B.2 A Model with Labor Externalities}

\section{B.2.1 The Model Economy}

A private agent in the economy has has the following preferences

$$
U=(1-\sigma)^{-1} \int_{0}^{\infty} e^{-\rho t}\left[C(t) \exp \left(-N(t) \bar{N}(t)^{-\gamma_{N}}\right)\right]^{1-\sigma} d t
$$

over time paths for individual consumption $C$, hours worked $N$, and aggregate hours $\bar{N}$. We assume this functional form for the utility to preserve balanced growth. Also, $\rho=1 / \beta-1>0$ and $\sigma \geq 0$, where $\beta$ is the subjective discount factor and $\sigma$ is the inverse of the intertemporal elasticity of substitution. $\gamma_{\bar{N}} \in(-\infty, 1]$ measures the degree of the labor externality

The private agent is subject to the following constraints:

$$
C(t)+I(t)=K(t)^{\alpha}(A(t) N(t))^{1-\alpha}
$$




$$
\dot{K}(t)=I(t)-\delta K(t)-\frac{\psi_{I}}{2}\left(1-\frac{I(t)}{\delta K(t)}\right)^{2} I(t)
$$

Here $K, I$ and $A$ represent capital, investment and the level of technology. The path of technology and the initial capital stock are exogenous. The depreciation rate, $\delta$, and the elasticity of output with respect to capital, $\alpha$, both lie between zero and one. $\psi_{I} \in[0, \infty)$ gives a measure of the magnitude of the convex investment adjustment costs.

We include convex adjustment costs as a way to generate an increase in the shadow value of investment in response to a news technology shock. In the basic model we achieved this by a low IES. Unfortunately, in a model with labor externalities a low IES leads to a non-stable solution.

Next, let us define the exogenous processes - the technology news shock. The private agents have perfect foresight, with

$$
A(t)=\left\{\begin{array}{ccrl}
\bar{A} & \text { for } t & \in[0, T) \\
\tilde{A}=1.01 \times \bar{A} & t \geq T
\end{array}\right.
$$

For the contemporaneous improvements case $T=0$ in the above specification.

\section{B.2.2 The Model Economy's First Order Conditions}

The private agents choose $C, I, K$, and $N$ to maximize $U$ subject to (77) and (78) taking as given the initial condition $K(0)$ and time path of technology. We can express the problem as a current value Hamiltonian:

$H=C^{1-\sigma} \exp \left[-(1-\sigma) N \bar{N}^{-\gamma_{\bar{N}}^{L E}}\right]+\Lambda\left(I-\delta K-\frac{\psi_{I}}{2}\left(1-\frac{I}{\delta K}\right)^{2} I\right)+\Phi\left(K^{\alpha}(A N)^{1-\alpha}-F(C, I)\right)$

The first-order necessary conditions at an interior solution in a symmetric equilibrium satisfy

$$
\begin{gathered}
-\frac{U_{N}}{U_{C}}=(1-\alpha) \frac{F}{N} \\
U_{C}=\Lambda\left[1-\frac{\psi_{I}}{2}\left(1-\frac{I}{\delta K}\right)^{2}+\psi_{I}\left(1-\frac{I}{\delta K}\right) \frac{I}{\delta K}\right]
\end{gathered}
$$




$$
\frac{\dot{\Lambda}}{\Lambda}-\rho=\delta+\psi_{I}\left(1-\frac{I}{\delta K}\right)\left(\frac{I}{\delta K}\right)^{2}-\alpha \frac{F}{K} \frac{U_{C}}{\Lambda}
$$

along with our initial condition on capital and a transversality condition on $\Lambda$.

Equation (79) is the intratemporal Euler equation between consumption and labor hours, equation (80) is the intratemporal Euler equation between consumption and investment, and equation (81) is the optimal capital accumulation equation.

\section{B.2.3 The Model Economy Log Linearized and Simplified}

Given the first order conditions in the previous section our model economy can be described by the following five log linearized equations:

$$
\begin{gathered}
\left(1-s_{I}\right) c+s_{I} i=\alpha k+(1-\alpha)(a+n) \\
\frac{s_{I}}{1-\gamma_{N}}(i-c)=n \\
\lambda=-\sigma c-\frac{(1-\sigma)(1-\alpha)}{\left(1-s_{I}\right)} n+\psi_{I}(i-k) \\
\dot{k}=\delta(i-k) \\
\dot{\lambda}=-(\rho+\delta)\left[\left(1-s_{I}\right)(c-i)+i-k\right]+\rho \psi_{I}(i-k)
\end{gathered}
$$

Here, $s_{I}=\frac{\alpha \delta}{\rho+\delta}$. Notice that the term $\gamma_{N}$ only enters into equation (83).

We can substitute (83) into (82) to get the consumption-investment production frontier $\left(L_{1}\right.$ line):

$$
\left(1-\phi_{I}^{L E}\right) c+\phi_{I}^{L E} i=\alpha k+(1-\alpha) a
$$


Here, $\phi_{I}^{L E}=\left(1-\frac{1-\alpha}{1-\gamma_{N}}\right) s_{I}$

We can also substitute (83) into (84) to get the consumption-investment euler equation $\left(L_{2}\right.$ line):

$$
\left(\gamma_{I}^{L E}+\psi_{I}\right) i-\left(\sigma+\gamma_{I}^{L E}\right) c=\lambda
$$

Here, $\gamma_{I}^{L E}=-\frac{(1-\alpha)(1-\sigma) s_{I}}{\left(1-s_{I}\right)\left(1-\gamma_{N}\right)}$.

Equations (87) and (88) now give us a system of equations in $i$ and $c$ (treating $\lambda, k$, and $a$ as exogenous).

We also solve the system of equations above for $c, i, n, \dot{k}$, and $\dot{\lambda}$, assuming as given the state variable $\lambda$ and $k$, and the exogenous variable $a$ :

$$
\begin{aligned}
& c=\tau_{c, k}^{L E} k+\tau_{c, \lambda}^{L E} \lambda+\tau_{c, a}^{L E} a \\
& i=\tau_{i, k}^{L E} k+\tau_{i, \lambda}^{L E} \lambda+\tau_{i, a}^{L E} a \\
& n=\tau_{n, k}^{L E} k+\tau_{n, \lambda}^{L E} \lambda+\tau_{n, a}^{L E} a \\
& \dot{k}=\Gamma_{k, k}^{L E} k+\Gamma_{k, \lambda}^{L E} \lambda+b_{k, a} a \\
& \dot{\lambda}=\Gamma_{\lambda, k}^{L E} k+\Gamma_{\lambda, \lambda}^{L E} \lambda+b_{\lambda, a}^{L E} a
\end{aligned}
$$


where,

$$
\begin{array}{ll}
\tau_{c, k}^{L E}=\frac{\partial c}{\partial k}=\frac{\gamma_{I, L E}^{L E} \alpha+\psi_{I}\left(\alpha-\phi_{I, L E}^{L E}\right)}{\phi_{I, L E}^{L E} \sigma+\gamma_{I, L E}^{L E}+\psi_{I}\left(1-\phi_{I, L E}^{L E}\right)} & \Gamma_{k, k}^{L E}=\frac{\partial \dot{k}}{\partial k}=\frac{-\delta\left((1-\alpha) \gamma_{I, L E}^{L E}+\phi_{I, L E}^{L E} \sigma-\alpha \sigma\right)}{\phi_{I, L E}^{L E} \sigma+\gamma_{I, L E}^{L E}+\psi_{I}\left(1-\phi_{I, L E}^{L E}\right)} \\
\tau_{c, \lambda}^{L E}=\frac{\partial c}{\partial \lambda}=\frac{-\phi_{I, L E}^{L E}}{\phi_{I, L E}^{L E} \sigma+\gamma_{I, L E}^{L E}+\psi_{I}\left(1-\phi_{I, L E}^{L E}\right)} & \Gamma_{k, \lambda}^{L E}=\frac{\partial \dot{k}}{\partial \lambda}=\frac{\delta\left(1-\phi_{I, L E}^{L E}\right)}{\phi_{I, L E}^{L E} \sigma+\gamma_{I, L E}^{L E}+\psi_{I}\left(1-\phi_{I, L E}^{L E}\right)} \\
\tau_{c, a}^{L E}=\frac{\partial c}{\partial a}=\frac{\left(\gamma_{I, L E}^{L E}+\psi_{I}\right)(1-\alpha)}{\phi_{I, L E}^{L E} \sigma+\gamma_{I, L E}^{L E}+\psi_{I}\left(1-\phi_{I, L E}^{L E}\right)} & \Gamma_{\lambda, k}^{L E}=\frac{\partial \dot{\lambda}}{\partial k}=\frac{(\rho+\delta)\left((1-\alpha) \gamma_{I, L E}^{L E}+\phi_{I, L E}^{L E} \sigma-\alpha \sigma+\alpha \sigma\left(1-s_{I}\right)\right)}{\phi_{I, L E}^{L E} \sigma+\gamma_{I, L E}^{L E}+\psi_{I}\left(1-\phi_{I, L E}^{L E}\right)} \\
\tau_{i, k}^{L E}=\frac{\partial i}{\partial k}=\frac{\alpha\left(\gamma_{I, L E}^{L E}+\sigma\right)+\psi_{I}\left(1-\phi_{I, L E}^{L E}\right)}{\phi_{I, L E}^{L E} \sigma+\gamma_{I, L E}^{L E}+\psi_{I}\left(1-\phi_{I, L E}^{L E}\right)} & +\frac{\psi_{I}^{L E}\left[(\rho+\delta)\left(1-S_{I}\right)(1-\alpha)-\rho\left((1-\alpha) \gamma_{I}^{L E}+\left(\phi_{I}^{L E}-\alpha\right) \sigma\right)\right]}{\phi_{I}^{L E} \sigma+\gamma_{I}^{L E}+\psi_{I}\left(1-\phi_{I}^{L E}\right)} \\
\tau_{i, \lambda}^{L E}=\frac{\partial i}{\partial \lambda}=\frac{\Gamma_{\lambda, \lambda}^{L E}}{\phi_{I}^{L E} \sigma+\gamma_{I}^{L E}+\psi_{I}\left(1-\phi_{I}^{L E}\right)} & \frac{\partial \dot{\lambda}}{\partial \lambda}=\frac{(\rho+\delta)\left(\phi_{I}^{L E}-s_{I}\right)+\rho \psi_{I}\left(1-\phi_{I}^{L E}\right)}{\phi_{I}^{L E} \sigma+\gamma_{I}^{L E}+\psi_{I}\left(1-\phi_{I}^{L E}\right)} \\
\tau_{i, a}^{L E}=\frac{\partial i}{\partial a}=\frac{(1-\alpha)\left(\gamma_{I}^{L E}+\sigma\right)}{\phi_{I}^{L E} \sigma+\gamma_{I}^{L E}+\psi_{I}\left(1-\phi_{I}^{L E}\right)} & b_{k, a}^{L E}=\frac{\partial \dot{k}}{\partial a}=\frac{\delta\left(\gamma_{I}^{L E}+\sigma\right)(1-\alpha)}{\phi_{I}^{L E} \sigma+\gamma_{I}^{L E}+\psi_{I}\left(1-\phi_{I}^{L E}\right)} \\
\tau_{n, k}^{L E}=\frac{\partial n}{\partial k}=\frac{1}{1-\gamma_{N}} \frac{s_{I}\left(\alpha \sigma+\psi_{I}(1-\alpha)\right)}{\phi_{I}^{L E} \sigma+\gamma_{I}^{L E}+\psi_{I}\left(1-\phi_{I}^{L E}\right)} & b_{\lambda, a}^{L E}=\frac{\partial \dot{\lambda}}{\partial a}=\frac{-(\rho+\delta)(1-\alpha)\left(\sigma s_{I}+\gamma_{I}^{L E}\right)}{\phi_{I}^{L E} \sigma+\gamma_{I}^{L E}+\psi_{I}\left(1-\phi_{I}^{L E}\right)} \\
\tau_{n, \lambda}^{L E}=\frac{\partial n}{\partial \lambda}=\frac{1}{1-\gamma_{N}} \frac{s_{I}^{L E}}{\phi_{I}^{L E} \sigma+\gamma_{I}^{L E}+\psi_{I}\left(1-\phi_{I}^{L E}\right)} & +\frac{\psi_{I}(1-\alpha)\left[\rho\left(\sigma+\gamma_{I}^{L E}\right)-(\rho+\delta)\left(1-S_{I}\right)\right]}{\phi_{I}^{L E} \sigma+\gamma_{I}^{L E}+\psi_{I}\left(1-\phi_{I}^{L E}\right)} \\
\tau_{n, a}^{L E}=\frac{\partial n}{\partial a}=\frac{1}{1-\gamma_{N}} \frac{s_{I}(1-\alpha)\left(\sigma-\psi_{I}\right)}{\phi_{I}^{L E} \sigma+\gamma_{I}^{L E}+\psi_{I}\left(1-\phi_{I}^{L E}\right)} &
\end{array}
$$

Recall: $s_{I}=\frac{\alpha \delta}{\rho+\delta}, \phi_{I}^{L E}=\left(1-\frac{1-\alpha}{1-\gamma_{N}}\right) s_{I}$, and $\gamma_{I}^{L E}=-\frac{(1-\alpha)(1-\sigma) s_{I}}{\left(1-s_{I}\right)\left(1-\gamma_{N}\right)}$

\section{B.2.4 The Dynamic System}

The general solution to the dynamic system remains the same as before, but now with different coefficient values for $\tau_{x, x}^{L E}$ 's, $\Gamma_{x, x}^{L E}$ 's and $b_{x, x}$ 's. The new values for $\tau_{x, x}^{L E}$ 's, $\Gamma_{x, x}^{L E}$ 's and $b_{x, x}$ 's are given above. The solution to the dynamic system is:

$$
\lambda(0)=-\left[\frac{\Gamma_{k, \lambda}^{L E} b_{\lambda, a}^{L E}+\left(\mu_{2}^{L E}-\Gamma_{\lambda, \lambda}^{L E}\right) b_{k, a}^{L E}}{\Gamma_{k, \lambda}^{L E} \mu_{2}^{L E}}\right] e^{-\mu_{2}^{L E} T}
$$




$$
\begin{gathered}
k(t)=\left\{\begin{array}{cc}
\frac{\Gamma_{k, \lambda}^{L E} \lambda(0)}{\mu_{1}^{L E}-\mu_{2}^{L E}} e^{\mu_{1}^{L E} t}+\frac{\Gamma_{k, \lambda}^{L E} \lambda(0)}{\mu_{2}^{L E}-\mu_{1}^{L E}} e^{\mu_{2}^{L E} t} & \text { for } t \in[0, T) \\
\frac{\Gamma_{k, \lambda}^{L E} \lambda(0)}{\mu_{1}^{L E} \mu_{2}^{L E}} e^{\mu_{1}^{L E} t}+\frac{\Gamma_{k, \lambda}^{L E} b_{\lambda, a}^{L E}-\Gamma_{\lambda, \lambda}^{L E} \mu_{k, a}^{L E}}{\mu_{1}^{L E} \mu_{2}^{L E}}+\frac{\Gamma_{k, \lambda}^{L E} b_{\lambda, a}^{L E}+\left(\mu_{1}^{L E}-\Gamma_{\lambda, \lambda}^{L E}\right) b_{k, a}^{L E}}{\mu_{1}^{L E}\left(\mu_{1}^{L E}-\mu_{2}^{L E}\right)} e^{\mu_{1}^{L E}(t-T)} & t \geq T
\end{array}\right. \\
\lambda(t)=\left\{\begin{array}{cc}
(93) \\
\frac{\left(\mu_{1}^{L E}-\Gamma_{k, k}^{L E}\right) \lambda(0)}{\mu_{1}^{L E}-\mu_{2}^{L E}} e^{\mu_{1}^{L E} t}+\frac{\left(\mu_{2}^{L E}-\Gamma_{k, k}^{L E}\right) \lambda(0)}{\mu_{2}^{L E}-\mu_{1}^{L E}} e^{\mu_{2}^{L E} t} & \text { for } t \in[0, T) \\
\frac{\left(\mu_{1}^{L E}-\Gamma_{k, k}^{L E}\right) \lambda(0)}{\mu_{1}^{L E}-\mu_{2}^{L E}} e^{\mu_{1}^{L E} t}+\frac{\Gamma_{\lambda, k}^{L E} b_{k, a}^{L E}-\Gamma_{k, k}^{L E} b_{\lambda, a}^{L E}}{\mu_{1}^{L E} \mu_{2}^{L E}}+\frac{\Gamma_{\lambda, k}^{L E} b_{k, a}^{L E}+\left(\mu_{1}^{L E}-\Gamma_{k, k}^{L E}\right) b_{\lambda, a}^{L E}}{\mu_{1}^{L E}\left(\mu_{1}^{L E}-\mu_{2}^{L E}\right)} e^{\mu_{1}^{L E}(t-T)} & t \geq T
\end{array}\right.
\end{gathered}
$$

Equations (89), (90), (91), (93), and (94), along with equation (92) give us a stable solution to our model economy for a $1 \%$ technology shock that occurs in period $T$.

\section{B.2.5 Proofs \& Expressions}

In this section we will sign the various expressions needed for Lemma 7-9 and Theorem 2.

First, let us recall the proof for Lemma 6. For consumption, investment and hours to comove at time zero (on impact of the news) we required $\gamma_{N}>\alpha$ which resulted in $\phi_{I}^{L E}<0$ and thus a positively sloped $L_{1}$ line. Also, by lemma 8 we know that for a stable solution we need $\psi_{I}>-\frac{\gamma_{I}^{L E}+\phi_{I}^{L E} \sigma}{1-\phi_{I}^{L E}}$. For this section we will assume both that $\gamma_{N}>\alpha$ and $\psi_{I}>-\frac{\gamma_{I}^{L E}+\phi_{I}^{L E} \sigma}{1-\phi_{I}^{L E}}$

Assumption: $\gamma_{N}>=\gamma_{N}^{*}=\alpha$ and $\psi_{I}>\psi_{I}^{+}=-\frac{\gamma_{I}^{L E}+\phi_{I}^{L E} \sigma}{1-\phi_{I}^{L E}}$

Lemma B.8: $\phi_{I, L E}^{L E} \sigma+\gamma_{I, L E}^{L E}+\psi_{I}\left(1-\phi_{I, L E}^{L E}\right)>0$

Proof. Follows trivially from our assumption that $\psi_{I}>-\frac{\gamma_{I}^{L E}+\phi_{I}^{L E} \sigma}{1-\phi_{I}^{L E}}$

Lemma B.9: $\tau_{c, k}^{L E}, \tau_{c, \lambda}^{L E}, \tau_{i, k}^{L E}, \tau_{i, \lambda}^{L E}, \tau_{n, k}^{L E}$, and $\tau_{n, \lambda}^{L E}$ are all positive 
Proof. This result follows trivially lemma B.8 and our assumption, $\gamma_{N}>\alpha$, which ensures $\phi_{I}^{L E}<0$.

Lemma B.10: $\Gamma_{k, \lambda}^{L E}$ and $\Gamma_{\lambda, \lambda}^{L E}$ are both positive.

Proof. $\Gamma_{k, \lambda}^{L E}>0$ follows trivially from lemma B.8 and our assumption, $\gamma_{N}>\alpha \Rightarrow \phi_{I}^{L E}<0$.

On the other hand $\Gamma_{\lambda, \lambda}^{L E}>0$ follows trivially from lemma B.8, and our assumptions, $\gamma_{N}>$ $\alpha \Rightarrow \phi_{I}^{L E}<0$ and $\psi_{I}>-\frac{\gamma_{I}^{L E}+\phi_{I}^{L E} \sigma}{1-\phi_{I}^{L E}}$.

\section{B.3 A Model with Forward Compatible Investment}

\section{B.3.1 The Model Economy}

A social planner has the following preferences

$$
U=(1-\sigma)^{-1} \int_{0}^{\infty} e^{-\rho t}[C(t) \exp (-N(t))]^{1-\sigma} d t
$$

over time paths for consumption $C$ and hours worked $N$. We assume this functional form for the utility to preserve balanced growth. Also, $\rho=1 / \beta-1>0$ and $\sigma \geq 0$.

The planner is subject to the following constraints:

$$
\begin{gathered}
F(C(t), I(t))=K(t)^{\alpha} N(t)^{1-\alpha} \\
\dot{K}(t)=Q(t) I(t)-\delta K(t)+\left(K(t)-e^{-\delta T} \bar{K}\right) P(\tilde{Q}, t, T, \epsilon)
\end{gathered}
$$

Here $K, I$ and $q$ represent capital, investment and the level of technology embodied in the capital created at a point in time. The path of technology and the initial capital stock are exogenous. The depreciation rate, $\delta$, and the elasticity of output with respect to capital, $\alpha$, both lie between zero and one. For $\epsilon \rightarrow 0, P(\tilde{Q}, t, T, \epsilon)$ represents the level of forward compatibility. We talk about this process in detail later. In short, this represents the idea that capital might embody technology that does not become useful until a future date forward compatibility of capital with future technology. 
Further, we assume:

$$
F(C, I) \equiv\left[\theta C^{v}+(1-\theta) I^{v}\right]^{1 / v}
$$

where $\theta \in(0,1)$ and $v \geq 1$. When $v=1$, the equation collapses to the standard neo-classical case, which has infinite substitutability between the two goods. As $v$ increases, the complementarity between the production of the two goods increases. If $v=\infty$, the production frontier takes a Leontief form.

Next, let us define the exogenous processes - the level of capital embodied technology and the process that defines forward compatibility. We will consider two types of capital embodied technology shocks that occur at time zero: contemporaneous improvements, i.e. a current shock, and news of future improvements, i.e. a news shock. For both types of shocks, suppose the capital stock is at an initial steady state consistent with a particular fixed and unchanged level of technology $\bar{q}$. In the case of the future shock, the planner again has perfect foresight, with

$$
Q(t)=\left\{\begin{array}{cc}
\bar{Q} & \text { for } t \in[0, T) \\
\tilde{Q}=1.01 \times \bar{Q} & t \geq T
\end{array}\right.
$$

For the contemporaneous improvements case $T=0$ in the above specification

The process that defines the forward compatibility is as follows:

$$
P(\tilde{Q}, t, T, \varepsilon)=\left\{\begin{array}{cc}
0 & \text { for } t \in[0, T) \cup(T+\epsilon, \infty) \\
\frac{\tau^{F C} \tilde{Q}}{\left(1-e^{-\epsilon}\right) \bar{Q}} & t \in[T, T+\epsilon]
\end{array}\right.
$$

$\tau^{F C} \in[0,1]$ here represents the degree of forward compatibility of the capital accumulated between time 0 and the current period for $\epsilon \rightarrow 0$. When $\tau^{F C}=0$, this capital embodies none of the technology that will be useful this period onwards. While when $\tau^{F C}=1$, this capital embodies all of the technology that will become useful from this period onwards. At time T, all future investment becomes more productive in augmenting the capital stock. This can be equivalently thought of as a fall in the price of investment. 


\section{B.3.2 The Model Economy's First Order Conditions}

The social planner chooses $C, I, K$, and $N$ to maximize $U$ subject to (95) and (96) taking as given the initial condition $K(0)$ and time path of technology and shocks to the capital stock. We can express the problem as a current value Hamiltonian:

$$
H=C^{1-\sigma} \exp [-(1-\sigma) N]+\Lambda\left(Q I-\delta K+P_{\varepsilon} \mathcal{K}\right)+\Phi\left(K^{\alpha} N^{1-\alpha}-F(C, I)\right)
$$

The first-order necessary conditions at an interior solution satisfy :

$$
\begin{gathered}
-\frac{U_{N}}{U_{C}}=(1-\alpha) \frac{F}{N}\left(F_{C}\right)^{-1} \\
\frac{U_{C}}{Q \Lambda}=\frac{F_{C}}{F_{I}} \\
\frac{\dot{\Lambda}}{\Lambda}-\rho=\delta-P_{\epsilon}-\alpha Q \frac{F}{K}\left(F_{I}\right)^{-1}
\end{gathered}
$$

along with our initial condition on capital and a transversality condition on $\Lambda$.

\section{B.3.3 The Model Economy Log Linearized and Simplified}

Given the first order conditions in the previous section our model economy can be described by the following five log linearized equations:

$$
\begin{gathered}
\left(1-s_{I}\right) c+s_{I} i=\alpha k+(1-\alpha) n \\
v s_{I}(i-c)=n \\
\lambda+q=(1-v)(c-i)-\sigma c-\frac{(1-\sigma)(1-\alpha)}{\left(1-s_{I}\right)} n \\
\dot{k}=\delta(q+i-k)+\left(1-e^{\delta T}\right) p_{\epsilon}
\end{gathered}
$$




$$
\dot{\lambda}=-p_{\epsilon}-(\rho+\delta)\left[v\left(1-s_{I}\right)(c-i)+q+i-k\right]
$$

Here, $s_{I}=\frac{\alpha \delta}{\rho+\delta}$.

We can substitute (101) into (100) to get:

$$
\left(1-\phi_{I}^{F C}\right) c+\phi_{I}^{F C} i=\alpha k
$$

Here, $\phi_{I}^{F C}=(1-(1-\alpha) v) s_{I}$

We can also substitute (101) into (102) to get:

$$
\gamma_{I}^{F C} i-\left(\sigma+\gamma_{I}^{F C}\right) c=\lambda+q
$$

Here, $\gamma_{I}^{F C}=(v-1)-\frac{v(1-\alpha)(1-\sigma) s_{I}}{\left(1-s_{I}\right)}$.

Equations (105) and (106) now give us a system of equations in $i$ and $c$ (treating $\lambda, k$, and $q$ as exogenous). Solving this system, we get:

$$
\begin{gathered}
c=\left(\frac{\phi_{I}^{F C}}{\phi_{I}^{F C} \sigma+\gamma_{I}^{F C}}\right)\left(\frac{\gamma_{I}^{F C} \alpha}{\phi_{I}^{F C}} k-\lambda-q\right) \\
i=\left(\frac{\gamma_{I}^{F C}+\sigma}{\phi_{I}^{F C} \sigma+\gamma_{I}^{F C}}\right) \alpha k+\left(\frac{1-\phi_{I}^{F C}}{\phi_{I}^{F C} \sigma+\gamma_{I}^{F C}}\right)(\lambda+q)
\end{gathered}
$$

Further, substituting these above equations into (101), we also get an expression for $n$ in terms of $\lambda, k$, and $q$ :

$$
n=\frac{v s_{I}}{\phi_{I}^{F C}}\left(\left(1-\frac{\gamma_{I}^{F C}}{\phi_{I}^{F C} \sigma+\gamma_{I}^{F C}}\right) \alpha k+\frac{\phi_{I}^{F C}}{\phi_{I}^{F C} \sigma+\gamma_{I}^{F C}}(\lambda+q)\right)
$$

Finally, we can also simplify our two dynamic equations (103) and (104) in terms of $\lambda, k$, and $q$, by using (107), (108), and (109): 


$$
\begin{aligned}
\dot{k}= & \frac{\delta}{\phi_{I}^{F C} \sigma+\gamma_{I}^{F C}}\left[-\left((1-\alpha) \gamma_{I}^{F C}+\phi_{I}^{F C} \sigma-\alpha \sigma\right) k+\left(1-\phi_{I}^{F C}\right) \lambda+\left(1-\phi_{I}^{F C}+\phi_{I}^{F C} \sigma+\gamma_{I}^{F C}\right) q\right] \\
& +\left(1-e^{-\delta T}\right) p_{\epsilon}
\end{aligned}
$$

$$
\begin{aligned}
\dot{\lambda}= & -p_{\epsilon}+\frac{\rho+\delta}{\phi_{I}^{F C} \sigma+\gamma_{I}^{F C}}\left[\left((1-\alpha) \gamma_{I}^{F C}+\phi_{I}^{F C} \sigma-\alpha \sigma+\alpha \sigma v\left(1-s_{I}\right)\right) k\right]+ \\
& \frac{\rho+\delta}{\phi_{I}^{F C} \sigma+\gamma_{I}^{F C}}\left[\left(\phi_{I}^{F C}-\left(1-v\left(1-s_{I}\right)\right)\right) \lambda+\left(\phi_{I}^{F C}-\left(1-v\left(1-s_{I}\right)\right)-\phi_{I}^{F C} \sigma-\gamma_{I}^{F C}\right) q\right]
\end{aligned}
$$

Equations (107) - (111) now give us a simplified system of equations that define a dynamic stochastic general equilibrium for our model economy. For ease of use in this appendix we take this one step further and rewrite these equations as follows:

$$
\begin{gathered}
c=\tau_{c, k}^{F C} k+\tau_{c, \lambda}^{F C} \lambda+\tau_{c, q}^{F C} q \\
i=\tau_{i, k}^{F C} k+\tau_{i, \lambda}^{F C} \lambda+\tau_{i, q}^{F C} q \\
n=\tau_{n, k}^{F C} k+\tau_{n, \lambda}^{F C} \lambda+\tau_{n, q}^{F C} q \\
\dot{k}=\Gamma_{k, k}^{F C} k+\Gamma_{k, \lambda}^{F C} \lambda+b_{k, q} q+b_{k, p}^{F C} p_{\epsilon} \\
\dot{\lambda}=\Gamma_{\lambda, k}^{F C} k+\Gamma_{\lambda, \lambda}^{F C} \lambda+b_{\lambda, q} q+b_{\lambda, p}^{F C} p_{\epsilon}
\end{gathered}
$$


Here,

$$
\begin{array}{ll}
\tau_{c, k}^{F C}=\frac{\partial c}{\partial k}=\frac{\gamma_{I}^{F C} \alpha}{\phi_{I}^{F C} \sigma+\gamma_{I}^{F C}} & \Gamma_{k, k}^{F C}=\frac{\partial \dot{k}}{\partial k}=\frac{-\delta\left((1-\alpha) \gamma_{I}^{F C}+\phi_{I}^{F C} \sigma-\alpha \sigma\right)}{\phi_{I}^{F C} \sigma+\gamma_{I}^{F C}} \\
\tau_{c, \lambda}^{F C}=\frac{\partial c}{\partial \lambda}=\frac{-\phi_{I}^{F C}}{\phi_{I}^{F C} \sigma+\gamma_{I}^{F C}} & \Gamma_{k, \lambda}^{F C}=\frac{\partial \dot{k}}{\partial \lambda}=\frac{\delta\left(1-\phi_{I}^{F C}\right)}{\phi_{I}^{F C} \sigma+\gamma_{I}^{F C}} \\
\tau_{c, q}^{F C}=\frac{\partial c}{\partial q}=\frac{-\phi_{I}^{F C}}{\phi_{I}^{F C} \sigma+\gamma_{I}^{F C}} & \Gamma_{\lambda, k}^{F C}=\frac{\partial \dot{\lambda}}{\partial k}=\frac{(\rho+\delta)\left((1-\alpha) \gamma_{I}^{F C}+\phi_{I}^{F C} \sigma-\alpha \sigma+\alpha \sigma v\left(1-s_{I}\right)\right)}{\phi_{I}^{F C} \sigma+\gamma_{I}^{F C}} \\
\tau_{i, k}^{F C}=\frac{\partial i}{\partial k}=\frac{\alpha\left(\gamma_{I}^{F C}+\sigma\right)}{\phi_{I}^{F C} \sigma+\gamma_{I}^{F C}} & \Gamma_{\lambda, \lambda}^{F C}=\frac{\partial \dot{\lambda}}{\partial \lambda}=\frac{(\rho+\delta)\left(\phi_{I}^{F C}-\left(1-v\left(1-s_{I}\right)\right)\right)}{\phi_{I}^{F C} \sigma+\gamma_{I}^{F C}} \\
\tau_{i, \lambda}^{F C}=\frac{\partial i}{\partial \lambda}=\frac{1-\phi_{I}^{F C}}{\phi_{I}^{F C} \sigma+\gamma_{I}^{F C}} & b_{k, q}=\frac{\partial \dot{k}}{\partial q}=\delta\left(\frac{1-\phi_{I}^{F C}}{\left.\phi_{I}^{F C} \sigma+\gamma_{I}^{F C}+1\right)}\right. \\
\tau_{i, q}^{F C}=\frac{\partial i}{\partial q}=\frac{1-\phi_{I}^{F C}}{\phi_{I}^{F C} \sigma+\gamma_{I}^{F C}} & b_{\lambda, q}=\frac{\partial \dot{\lambda}}{\partial q}=\frac{(\rho+\delta)\left(\phi_{I}^{F C}-\left(1-v\left(1-s_{I}\right)\right)-\phi_{I}^{F C} \sigma-\gamma_{I}^{F C}\right)}{\phi_{I}^{F C} \sigma+\gamma_{I}^{F C}} \\
\tau_{n, k}^{F C}=\frac{\partial n}{\partial k}=\frac{v s_{I} \alpha \sigma}{\phi_{I}^{F C} \sigma+\gamma_{I}^{F C}} & b_{k, p}^{F C}=\frac{\partial k}{\partial p_{\epsilon}}=\left(1-e^{-\delta T}\right) \\
\tau_{n, \lambda}^{F C}=\frac{\partial n}{\partial \lambda}=\frac{v s_{I}}{\phi_{I}^{F C} \sigma+\gamma_{I}^{F C}} & b_{\lambda, p}^{F C}=\frac{\partial \lambda}{\partial p_{\epsilon}}=-1 \\
\tau_{n, q}^{F C}=\frac{\partial n}{\partial q}=\frac{v s_{I}}{\phi_{I}^{F C} \sigma+\gamma_{I}^{F C}} &
\end{array}
$$

Recall: $s_{I}=\frac{\alpha \delta}{\rho+\delta}, \phi_{I}^{F C}=(1-(1-\alpha) v) s_{I}$, and $\gamma_{I}^{F C}=(v-1)-\frac{v(1-\alpha)(1-\sigma) s_{I}}{\left(1-s_{I}\right)}$

\section{B.3.4 The Dynamic System}

Let us now look at the dynamic system:

$$
\left[\begin{array}{l}
\dot{\lambda}(t) \\
\dot{k}(t)
\end{array}\right]=\left[\begin{array}{cc}
\Gamma_{\lambda, \lambda}^{F C} & \Gamma_{\lambda, k}^{F C} \\
\Gamma_{k, \lambda}^{F C} & \Gamma_{k, k}^{F C}
\end{array}\right]\left[\begin{array}{c}
\lambda(t) \\
k(t)
\end{array}\right]+\left[\begin{array}{c}
b_{\lambda, q} \\
b_{k, q}
\end{array}\right] q(t)+\left[\begin{array}{c}
b_{\lambda, p}^{F C} \\
b_{k, p}^{F C}
\end{array}\right] p_{\epsilon}
$$

Let $\mu_{1}^{F C}$ and $\mu_{2}^{F C}$ represent the two eigenvalues of $\Gamma^{F C}$. From the production complementarity model, we know that:

1. $\mu_{1}^{F C}+\mu_{2}^{F C}>0$

2. $\mu_{1}^{F C} \mu_{2}^{F C}<0$ 
Therefore, one of the eigenvalues must be negative and the other positive. Without loss of generality, we will assume henceforth that $\mu_{1}^{F C}<0$ and $\mu_{2}^{F C}>0$.

We now introduce a permanent increase in technology in period $T$. Specifically,

$$
q(t)=w(t)=\left\{\begin{array}{cc}
0 & \text { for } t \in[0, T) \\
1 & t \geq T
\end{array}\right.
$$

Further, let us define the shock to capital stock (we will take the limits later):

$$
w_{p_{\epsilon}}(t)=\left\{\begin{array}{cc}
0 & \text { for } t \in[0, T) \cup(T+\epsilon, \infty) \\
\frac{\tau^{F C}}{1-e^{-\epsilon}} & t \in[T, T+\epsilon]
\end{array}\right.
$$

To analyze the resulting system, it will be useful to introduce the Laplace transform operator.

The Laplace transform of a function $p(t)$ is:

$$
\mathcal{L}[p(t)]=\bar{P}(s)=\int_{0}^{\infty} e^{-s t} p(t) d t
$$

We will use $\bar{P}$ rather than $P$ to distinguish the Laplace transform of the log deviation of a variables from the level of said variable.

Moreover, we know from Theorem 6.3 from Boyce and Diprima (1969), that

$$
\mathcal{L}\left[p^{\prime}(t)\right]=s \mathcal{L}(p(t))-p(0)
$$

Taking the Laplace transform of the differential equations in $\left[\begin{array}{ll}\lambda & k\end{array}\right]^{\prime}$ and applying this theorem, we get:

$$
\left[\begin{array}{l}
\bar{\Lambda}(s) \\
\bar{K}(s)
\end{array}\right]=\left(s I-\Gamma^{F C}\right)^{-1}\left\{\left[\begin{array}{l}
\lambda(0) \\
k(0)
\end{array}\right]+\left[\begin{array}{c}
b_{\lambda, q} \\
b_{k, q}
\end{array}\right] W(s)+\left[\begin{array}{c}
b_{\lambda, p}^{F C} \\
b_{k, p}^{F C}
\end{array}\right] W_{p_{\epsilon}}(s)\right\}
$$

Given (116), it can be shown that

$$
\bar{W}(s)=\mathcal{L}[w(t)]=\frac{1}{s} e^{-s T}
$$


Given (117), it can be shown that

$$
\bar{W}_{p_{\epsilon}}(s)=\mathcal{L}\left[w_{\epsilon_{\kappa}}(t)\right]=\tau^{F C} \frac{e^{-s T}-e^{-s(T+\epsilon)}}{s\left(1-e^{-\epsilon}\right)}
$$

Rewriting equation (118), we get:

$\left[\begin{array}{c}\bar{\Lambda}(s) \\ \bar{K}(s)\end{array}\right]=\frac{1}{\left(s-\mu_{1}^{F C}\right)\left(s-\mu_{2}^{F C}\right)}\left[\begin{array}{cc}s-\Gamma_{k, k}^{F C} & \Gamma_{\lambda, k}^{F C} \\ \Gamma_{k, \lambda}^{F C} & s-\Gamma_{\lambda, \lambda}^{F C}\end{array}\right]\left\{\left[\begin{array}{c}\lambda(0) \\ k(0)\end{array}\right]+\left[\begin{array}{c}b_{\lambda, q} \\ b_{k, q}\end{array}\right] W(s)+\left[\begin{array}{c}b_{\lambda, p}^{F C} \\ b_{k, p}^{F C}\end{array}\right] W_{p_{\epsilon}}(s)\right\}$

Remember from the previous section, $\mu_{1}^{F C}$ and $\mu_{2}^{F C}$ are the eigenvalues of $\Gamma^{F C}$, and $\mu_{1}^{F C}<0$ and $\mu_{2}^{F C}>0$.

The lower row of (119) gives us:

$$
\begin{aligned}
\bar{K}(s)= & \frac{\Gamma_{k, \lambda}^{F C} \lambda(0)+\left(s-\Gamma_{\lambda, \lambda}^{F C}\right) k(0)}{\left(s-\mu_{1}^{F C}\right)\left(s-\mu_{2}^{F C}\right)}+\left[\frac{\Gamma_{k, \lambda}^{F C} b_{\lambda, q}+\left(s-\Gamma_{\lambda, \lambda}^{F C}\right) b_{k, q}}{s\left(s-\mu_{1}^{F C}\right)\left(s-\mu_{2}^{F C}\right)}\right] e^{-s T} \\
& +\left[\frac{\Gamma_{k, \lambda}^{F C} b_{\lambda, p}^{F C}+\left(s-\Gamma_{\lambda, \lambda}^{F C}\right) b_{k, p}^{F C}}{s\left(s-\mu_{1}^{F C}\right)\left(s-\mu_{2}^{F C}\right)}\right] \frac{\tau^{F C}\left(e^{-s T}-e^{-s(T+\epsilon)}\right)}{1-e^{-\epsilon}}
\end{aligned}
$$

Next, we take the inverse Laplace transform of $K(s)$ to recover $k$ as a function of time. After some algebra,

$$
\begin{aligned}
k(t)= & \frac{\Gamma_{k, \lambda}^{F C} \lambda(0)}{\mu_{1-}^{F C} \mu_{2}^{F C}} e^{\mu_{1}^{F C} t}+\frac{\Gamma_{k, \lambda}^{F C} \lambda(0)}{\mu_{2-}^{F C} \mu_{1}^{F C}} e^{\mu_{2}^{F C} t}+\frac{\left(\mu_{1}^{F C}-\Gamma_{\lambda, \lambda}^{F C}\right) k(0)}{\mu_{1}^{F C}-\mu_{2}^{F C}} e^{\mu_{1}^{F C} t}+\frac{\left(\mu_{2}^{F C}-\Gamma_{\lambda, \lambda}^{F C}\right) k(0)}{\mu_{2}^{F C}-\mu_{1}^{F C}} e^{\mu_{2}^{F C} t} \\
& +u_{T}(t)\left(\frac{\Gamma_{k, \lambda}^{F C} b_{\lambda, q}-\Gamma_{\lambda, \lambda}^{F C} b_{k, q}}{\mu_{1}^{F C} \mu_{2}^{F C}}\right) \\
& +u_{T}(t)\left(\frac{\Gamma_{k, \lambda}^{F C} b_{\lambda, q}+\left(\mu_{1}^{F C}-\Gamma_{\lambda, \lambda}^{F C}\right) b_{k, q}}{\mu_{1}^{F C}\left(\mu_{1}^{F C}-\mu_{2}^{F C}\right)} e^{\mu_{1}^{F C}(t-T)}\right) \\
& +u_{T}(t)\left(\frac{\Gamma_{k, \lambda}^{F C} b_{\lambda, q}+\left(\mu_{2}^{F C}-\Gamma_{\lambda, \lambda}^{F C}\right) b_{k, q}}{\mu_{2}^{F C}\left(\mu_{2}^{F C}-\mu_{1}^{F C}\right)} e^{\mu_{2}^{F C}(t-T)}\right)
\end{aligned}
$$




$$
\begin{aligned}
& +\frac{\tau^{F C}\left(u_{T}(t)-u_{T+\epsilon}(t)\right)}{1-e^{\epsilon}}\left(\frac{\Gamma_{k, \lambda}^{F C} b_{\lambda, p}^{F C}-\Gamma_{\lambda, \lambda}^{F C} b_{k, p}^{F C}}{\mu_{1}^{F C} \mu_{2}^{F C}}\right) \\
& +\frac{\tau^{F C}\left(u_{T}(t)-u_{T+\epsilon}(t) e^{-\mu_{1}^{F C} \epsilon}\right)}{1-e^{\epsilon}}\left(\frac{\Gamma_{k, \lambda}^{F C} b_{\lambda, p}^{F C}+\left(\mu_{1}^{F C}-\Gamma_{\lambda, \lambda}^{F C}\right) b_{k, p}^{F C}}{\mu_{1}^{F C}\left(\mu_{1}^{F C}-\mu_{2}^{F C}\right)} e^{\mu_{1}^{F C}(t-T)}\right) \\
& +\frac{\tau^{F C}\left(u_{T}(t)-u_{T+\epsilon}(t) e^{-\mu_{2}^{F C} \epsilon}\right)}{1-e^{\epsilon}}\left(\frac{\Gamma_{k, \lambda}^{F C} b_{\lambda, p}^{F C}+\left(\mu_{2}^{F C}-\Gamma_{\lambda, \lambda}^{F C}\right) b_{k, p}^{F C}}{\mu_{2}^{F C}\left(\mu_{2}^{F C}-\mu_{1}^{F C}\right)} e^{\mu_{2}^{F C}(t-T)}\right)
\end{aligned}
$$

where $u_{T}(t)$ is a step function that takes on a value of one for all $t \geq T$, and zero otherwise. Now taking the limit as $\epsilon \rightarrow 0$ we get:

$$
\begin{aligned}
k(t)= & \frac{\Gamma_{k, \lambda}^{F C} \lambda(0)}{\mu_{1-}^{F C} \mu_{2}^{F C}} e^{\mu_{1}^{F C} t}+\frac{\Gamma_{k, \lambda}^{F C} \lambda(0)}{\mu_{2-}^{F C} \mu_{1}^{F C}} e^{\mu_{2}^{F C} t}+\frac{\left(\mu_{1}^{F C}-\Gamma_{\lambda, \lambda}^{F C}\right) k(0)}{\mu_{1}^{F C}-\mu_{2}^{F C}} e^{\mu_{1}^{F C} t}+\frac{\left(\mu_{2}^{F C}-\Gamma_{\lambda, \lambda}^{F C}\right) k(0)}{\mu_{2}^{F C}-\mu_{1}^{F C}} e^{\mu_{2}^{F C} t} \\
& +u_{T}(t)\left(\frac{\Gamma_{k, \lambda}^{F C} b_{\lambda, q}-\Gamma_{\lambda, \lambda}^{F C} b_{k, q}}{\mu_{1}^{F C} \mu_{2}^{F C}}\right) \\
& +u_{T}(t)\left(\frac{\Gamma_{k, \lambda}^{F C}\left(b_{\lambda, q}+\tau^{F C} \mu_{1}^{F C} b_{\lambda, p}^{F C}\right)+\left(\mu_{1}^{F C}-\Gamma_{\lambda, \lambda}^{F C}\right)\left(b_{k, q}+\tau^{F C} \mu_{1}^{F C} b_{k, p}^{F C}\right)}{\mu_{1}^{F C}\left(\mu_{1}^{F C}-\mu_{2}^{F C}\right)} e^{\mu_{1}^{F C}(t-T)}\right) \\
& +u_{T}(t)\left(\frac{\Gamma_{k, \lambda}^{F C}\left(b_{\lambda, q}+\tau^{F C} \mu_{2}^{F C} b_{\lambda, p}^{F C}\right)+\left(\mu_{2}^{F C}-\Gamma_{\lambda, \lambda}^{F C}\right)\left(b_{k, q}+\tau^{F C} \mu_{2}^{F C} b_{k, p}^{F C}\right)}{\mu_{2}^{F C}\left(\mu_{2}^{F C}-\mu_{1}^{F C}\right)} e^{\mu_{2}^{F C}(t-T)}\right)
\end{aligned}
$$

Recall that we assume the initial capital stock is at the steady-state level associated with the pre-shock technology level. As such, $k(0)=0$ :

$$
\begin{aligned}
k(t)= & \frac{\Gamma_{k, \lambda}^{F C} \lambda(0)}{\mu_{1-}^{F C} \mu_{2}^{F C}} e^{\mu_{1}^{F C} t}+\frac{\Gamma_{k, \lambda}^{F C} \lambda(0)}{\mu_{2}^{F C} \mu_{1}^{F C}} e^{\mu_{2}^{F C} t}+ \\
& +u_{T}(t)\left(\frac{\Gamma_{k, \lambda}^{F C} b_{\lambda, q}-\Gamma_{\lambda, \lambda}^{F C} b_{k, q}}{\mu_{1}^{F C} \mu_{2}^{F C}}\right) \\
& +u_{T}(t)\left(\frac{\Gamma_{k, \lambda}^{F C}\left(b_{\lambda, q}+\tau^{F C} \mu_{1}^{F C} b_{\lambda, p}^{F C}\right)+\left(\mu_{1}^{F C}-\Gamma_{\lambda, \lambda}^{F C}\right)\left(b_{k, q}+\tau^{F C} \mu_{1}^{F C} b_{k, p}^{F C}\right)}{\mu_{1}^{F C}\left(\mu_{1}^{F C}-\mu_{2}^{F C}\right)} e^{\mu_{1}^{F C}(t-T)}\right) \\
& +u_{T}(t)\left(\frac{\Gamma_{k, \lambda}^{F C}\left(b_{\lambda, q}+\tau^{F C} \mu_{2}^{F C} b_{\lambda, p}^{F C}\right)+\left(\mu_{2}^{F C}-\Gamma_{\lambda, \lambda}^{F C}\right)\left(b_{k, q}+\tau^{F C} \mu_{2}^{F C} b_{k, p}^{F C}\right)}{\mu_{2}^{F C}\left(\mu_{2}^{F C}-\mu_{1}^{F C}\right)} e^{\mu_{2}^{F C}(t-T)}\right)
\end{aligned}
$$

This gives us the solution to a differential equation with one undetermined variable $\lambda(0)$. 
We now seek a path for $\left[\begin{array}{ll}\lambda & k\end{array}\right]^{\prime}$ that is not explosive. In order to achieve this, we choose $\lambda(0)$ such that the explosive root $\mu_{2}^{F C}$ is 'zeroed out' for all $t>T$. Otherwise, the path for $k(t)$ will be explosive. This restriction on $\lambda(0)$ is:

$$
\left(\frac{\Gamma_{k, \lambda}^{F C}}{\mu_{2}^{F C}-\mu_{1}^{F C}}\right) \lambda(0)=-\frac{\Gamma_{k, \lambda}^{F C}\left(b_{\lambda, q}+\tau^{F C} \mu_{2}^{F C} b_{\lambda, p}^{F C}\right)+\left(\mu_{2}^{F C}-\Gamma_{\lambda, \lambda}^{F C}\right)\left(b_{k, q}+\tau^{F C} \mu_{2}^{F C} b_{k, p}^{F C}\right)}{\mu_{2}^{F C}\left(\mu_{2}^{F C}-\mu_{1}^{F C}\right)} e^{-\mu_{2}^{F C} T}
$$

This can be re-written as:

$$
\lambda(0)=-\left[\frac{\Gamma_{k, \lambda}^{F C}\left(b_{\lambda, q}+\tau^{F C} \mu_{2}^{F C} b_{\lambda, p}^{F C}\right)+\left(\mu_{2}^{F C}-\Gamma_{\lambda, \lambda}^{F C}\right)\left(b_{k, q}+\tau^{F C} \mu_{2}^{F C} b_{k, p}^{F C}\right)}{\Gamma_{k, \lambda}^{F C} \mu_{2}^{F C}}\right] e^{-\mu_{2}^{F C} T}
$$

Let us also solve the second half of our laplace transform. This will allow us to study the path of $\lambda(t)$ over time. The first row of (119) gives us:

$$
\begin{aligned}
\bar{\Lambda}(s)= & \frac{\left(s-\Gamma_{k, k}^{F C}\right) \lambda(0)+\Gamma_{\lambda, k}^{F C} k(0)}{\left(s-\mu_{1}^{F C}\right)\left(s-\mu_{2}^{F C}\right)}+\left[\frac{\left(s-\Gamma_{k, k}^{F C}\right) b_{\lambda, q}+\Gamma_{\lambda, k}^{F C} b_{k, q}}{s\left(s-\mu_{1}^{F C}\right)\left(s-\mu_{2}^{F C}\right)}\right] e^{-s T} \\
& +\left[\frac{\left(s-\Gamma_{k, k}^{F C}\right) b_{\lambda, p}^{F C}+\Gamma_{\lambda, k}^{F C} b_{k, p}^{F C}}{s\left(s-\mu_{1}^{F C}\right)\left(s-\mu_{2}^{F C}\right)}\right] \frac{\tau^{F C}\left(-s T-e^{-s(T+\epsilon)}\right)}{1-e^{\epsilon}}
\end{aligned}
$$

Now we can take the inverse Laplace transform of $\Lambda(s)$ to recover $\lambda$ as a function of time. After some algebra, and then similar to before, taking the limit and setting $k(0)=0$ we get:

$$
\begin{aligned}
\lambda(t)= & \frac{\left(\mu_{1}^{F C}-\Gamma_{k, k}^{F C}\right) \lambda(0)}{\mu_{1-}^{F C} \mu_{2}^{F C}} e^{\mu_{1}^{F C} t}+\frac{\left(\mu_{2}^{F C}-\Gamma_{k, k}^{F C}\right) \lambda(0)}{\mu_{2-}^{F C} \mu_{1}^{F C}} e^{\mu_{2}^{F C} t} \\
& +u_{T}(t)\left(\frac{\Gamma_{\lambda, k}^{F C} b_{k, q}-\Gamma_{k, k}^{F C} b_{\lambda, q}}{\mu_{1}^{F C} \mu_{2}^{F C}}\right) \\
& +u_{T}(t)\left(\frac{\left(\mu_{1}^{F C}-\Gamma_{k, k}^{F C}\right)\left(b_{\lambda, q}+\tau^{F C} \mu_{1}^{F C} b_{\lambda, p}^{F C}\right)+\Gamma_{\lambda, k}^{F C}\left(b_{k, q}+\tau^{F C} \mu_{1}^{F C} b_{k, p}^{F C}\right)}{\mu_{1}^{F C}\left(\mu_{1}^{F C}-\mu_{2}^{F C}\right)} e^{\mu_{1}^{F C}(t-T)}\right) \\
& +u_{T}(t)\left(\frac{\left(\mu_{2}^{F C}-\Gamma_{k, k}^{F C}\right)\left(b_{\lambda, q}+\tau^{F C} \mu_{2}^{F C} b_{\lambda, p}^{F C}\right)+\Gamma_{\lambda, k}^{F C}\left(b_{k, q}+\tau^{F C} \mu_{2}^{F C} b_{k, p}^{F C}\right)}{\mu_{2}^{F C}\left(\mu_{2}^{F C}-\mu_{1}^{F C}\right)} e^{\mu_{2}^{F C}(t-T)}\right)
\end{aligned}
$$

Given that we choose a $\lambda(0)$ such that the explosive root $\mu_{2}^{F C}$ is 'zeroed out' for all $t>T$, 
we can simplify our equations for the time paths of $k(t)$ and $\lambda(t)$ to the following:

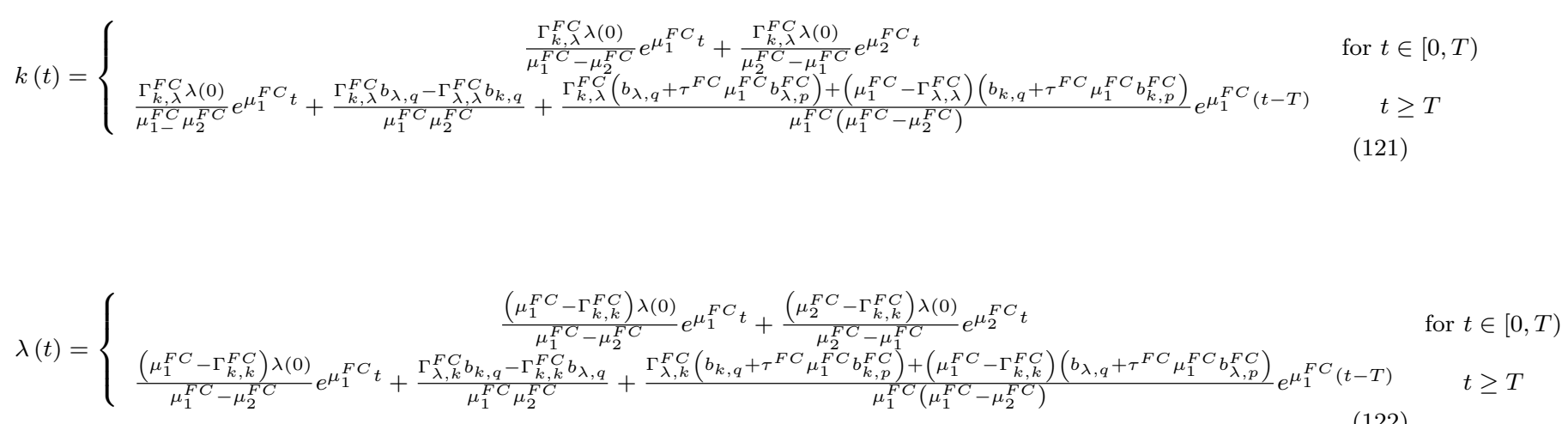

Equations (112), (113), (114), (121), and (122), along with equation (120) give us a stable solution to our model economy for a $1 \%$ technology shock that occurs in period $T$. Simultaneously in period $T$ the capital stock carried forward from period 0 (steady state) decreases by $\tau_{\kappa}^{F C}$ percent.

\section{B.4 A Model with Investment Adjustment Costs}

\section{B.4.1 The Model Economy}

A social planner has the following preferences

$$
U=(1-\sigma)^{-1} \int_{0}^{\infty} e^{-\rho t}[C(t) \exp (-N(t))]^{1-\sigma} d t
$$

over time paths for consumption $C$ and hours worked $N$. We assume this functional form for the utility to preserve balanced growth. Also, $\rho=1 / \beta-1>0$ and $\sigma \geq 0$, where $\beta$ is the subjective discount factor and $\sigma$ is the inverse of the intertemporal elasticity of substitution.

The planner is subject to the following constraints:

$$
\begin{gathered}
F[C(t), I(t)]=K(t)^{\alpha}(A(t) N(t))^{1-\alpha} \\
\dot{K}(t)=I(t)-\delta K(t)-\frac{\psi_{I}}{2}\left(1-\frac{I(t)}{\delta K(t)}\right)^{2} I(t)
\end{gathered}
$$

Here $K, I$ and $A$ represent capital, investment and the level of technology. The path of 
technology and the initial capital stock are exogenous. The depreciation rate, $\delta$, and the elasticity of output with respect to capital, $\alpha$, both lie between zero and one. $\psi_{I} \in[0, \infty)$ gives a measure of the magnitude of the convex investment adjustment costs.

Further, we assume:

$$
F(C, I) \equiv\left[\theta C^{v}+(1-\theta) I^{v}\right]^{1 / v}
$$

where $\theta \in(0,1)$ and $v \geq 1$. When $v=1$, the equation collapses to the standard neo-classical case, which has infinite substitutability between the two goods. As $v$ increases, the complementarity between the production of the two goods increases. If $v=\infty$, the production frontier takes a Leontief form.

Next, let us define the exogenous processes - the technology news shock. The planner again has perfect foresight, with

$$
A(t)=\left\{\begin{array}{cc}
\bar{A} & \text { for } t \in[0, T) \\
\tilde{A}=1.01 \times \bar{A} & t \geq T
\end{array}\right.
$$

For the contemporaneous improvements case $T=0$ in the above specification.

\section{B.4.2 The Model Economy's First Order Conditions}

The social planner chooses $C, I, K$, and $N$ to maximize $U$ subject to (123) and (124) taking as given the initial condition $K(0)$ and time path of technology. We can express the problem as a current value Hamiltonian:

$H=C^{1-\sigma} \exp [-(1-\sigma) N]+\Lambda\left(I-\delta K-\frac{\psi_{I}}{2}\left(1-\frac{I}{\delta K}\right)^{2} I\right)+\Phi\left(K^{\alpha}(A N)^{1-\alpha}-F(C, I)\right)$

The first-order necessary conditions at an interior solution satisfy :

$$
\begin{gathered}
-\frac{U_{N}}{U_{C}}=(1-\alpha) \frac{F}{N}\left(F_{C}\right)^{-1} \\
\frac{F_{I}}{F_{C}} U_{C}=\Lambda\left[1-\frac{\psi_{I}}{2}\left(1-\frac{I}{\delta K}\right)^{2}+\psi_{I}\left(1-\frac{I}{\delta K}\right) \frac{I}{\delta K}\right]
\end{gathered}
$$




$$
\frac{\dot{\Lambda}}{\Lambda}-\rho=\delta+\psi_{I}\left(1-\frac{I}{\delta K}\right)\left(\frac{I}{\delta K}\right)^{2}-\alpha \frac{F}{K} \frac{U_{C}}{\Lambda F_{C}}
$$

along with our initial condition on capital and a transversality condition on $\Lambda$.

Equation (125) is the intratemporal Euler equation between consumption and labor hours, equation (126) is the intratemporal Euler equation between consumption and investment, and equation (127) is the optimal capital accumulation equation.

\section{B.4.3 The Model Economy Log Linearized and Simplified}

Given the first order conditions in the previous section our model economy can be described by the following five log linearized equations:

$$
\begin{gathered}
\left(1-s_{I}\right) c+s_{I} i=\alpha k+(1-\alpha)(a+n) \\
v s_{I}(i-c)=n \\
\lambda=(1-v)(c-i)-\sigma c-\frac{(1-\sigma)(1-\alpha)}{\left(1-s_{I}\right)} n+\psi_{I}(i-k) \\
\dot{k}=\delta(i-k) \\
\dot{\lambda}=-(\rho+\delta)\left[v\left(1-s_{I}\right)(c-i)+i-k\right]+\rho \psi_{I}(i-k)
\end{gathered}
$$

Here, $s_{I}=\frac{\alpha \delta}{\rho+\delta}$.

We can substitute (129) into (128) to get the consumption-investment production frontier ( $L_{1}$ line):

$$
\left(1-\phi_{I}^{I N V}\right) c+\phi_{I}^{I N V} i=\alpha k+(1-\alpha) a
$$


Here, $\phi_{I}^{I N V}=(1-(1-\alpha) v) s_{I}$

We can also substitute (129) into (130) to get the consumption-investment euler equation ( $L_{2}$ line):

$$
\left(\gamma_{I}^{I N V}+\psi_{I}\right) i-\left(\sigma+\gamma_{I}^{I N V}\right) c=\lambda+\psi_{I} k
$$

Here, $\gamma_{I}^{I N V}=(v-1)-\frac{v(1-\alpha)(1-\sigma) s_{I}}{\left(1-s_{I}\right)}$.

Equations (133) and (134) now give us a system of equations in $i$ and $c$ (treating $\lambda, k$, and $a$ as exogenous).

We also solve the system of equations above for $c, i, n, \dot{k}$, and $\dot{\lambda}$, assuming as given the state variable $\lambda$ and $k$, and the exogenous variable $a$ :

$$
\begin{aligned}
& c=\tau_{c, k}^{I N V} k+\tau_{c, \lambda}^{I N V} \lambda+\tau_{c, a}^{I N V} a \\
& i=\tau_{i, k}^{I N V} k+\tau_{i, \lambda}^{I N V} \lambda+\tau_{i, a}^{I N V} a \\
& n=\tau_{n, k}^{I N V} k+\tau_{n, \lambda}^{I N V} \lambda+\tau_{n, a}^{I N V} a \\
& \dot{k}=\Gamma_{k, k}^{I N V} k+\Gamma_{k, \lambda}^{I N V} \lambda+b_{k, a}^{I N V} a \\
& \dot{\lambda}=\Gamma_{\lambda, k}^{I N V} k+\Gamma_{\lambda, \lambda}^{I N V} \lambda+b_{\lambda, a}^{I N V} a
\end{aligned}
$$


where,

$$
\begin{array}{lc}
\tau_{c, k}^{I N V}=\frac{\partial c}{\partial k}=\frac{\gamma_{I}^{I N V} \alpha+\psi_{I}\left(\alpha-\phi_{I}^{I N V}\right)}{\phi_{I}^{I N V} \sigma+\gamma_{I}^{I N V}+\psi_{I}\left(1-\phi_{I}^{I N V}\right)} & \Gamma_{k, k}^{I N V}=\frac{\partial \dot{k}}{\partial k}=\frac{-\delta\left((1-\alpha) \gamma_{I}^{I N V}+\phi_{I}^{I N V} \sigma-\alpha \sigma\right)}{\phi_{I}^{I N V} \sigma+\gamma_{I}^{I N V}+\psi_{I}\left(1-\phi_{I}^{I N V}\right)} \\
\tau_{c, \lambda}^{I N V}=\frac{\partial c}{\partial \lambda}=\frac{-\phi_{I}^{I N V}}{\phi_{I}^{I N V} \sigma+\gamma_{I}^{I N V}+\psi_{I}\left(1-\phi_{I}^{I N V}\right)} & \Gamma_{k, \lambda}^{I N V}=\frac{\partial \dot{k}}{\partial \lambda}=\frac{\delta\left(1-\phi_{I}^{I N V}\right)}{\phi_{I}^{I N V} \sigma+\gamma_{I}^{I N V}+\psi_{I}\left(1-\phi_{I}^{I N V}\right)} \\
\tau_{c, a}^{I N V}=\frac{\partial c}{\partial a}=\frac{\left(\gamma_{I}^{I N V}+\psi_{I}\right)(1-\alpha)}{\phi_{I}^{I N V} \sigma+\gamma_{I}^{I N V}+\psi_{I}\left(1-\phi_{I}^{I N V}\right)} & \Gamma_{\lambda, k}^{I N V}=\frac{\partial \dot{\lambda}}{\partial k}=\frac{(\rho+\delta)\left((1-\alpha) \gamma_{I}^{I N V}+\phi_{I}^{I N V} \sigma-\alpha \sigma+\alpha \sigma v\left(1-s_{I}\right)\right)}{\phi_{I}^{I N V} \sigma+\gamma_{I}^{I N V}+\psi_{I}\left(1-\phi_{I}^{I N V}\right)} \\
\tau_{i, k}^{I N V}=\frac{\partial i}{\partial k}=\frac{\alpha\left(\gamma_{I}^{I N V}+\sigma\right)+\psi_{I}\left(1-\phi_{I}^{I N V}\right)}{\phi_{I}^{I N V} \sigma+\gamma_{I}^{I N V}+\psi_{I}\left(1-\phi_{I}^{I N V}\right)} & +\frac{\psi_{I}\left[(\rho+\delta) v\left(1-S_{I}\right)(1-\alpha)-\rho\left((1-\alpha) \gamma_{I}^{I N V}+\left(\phi_{I}^{I N V}-\alpha\right) \sigma\right)\right]}{\phi_{I}^{I N V} \sigma+\gamma_{I}^{I N V}+\psi_{I}\left(1-\phi_{I}^{I N V}\right)} \\
\tau_{i, \lambda}^{I N V}=\frac{\partial i}{\partial \lambda}=\frac{1-\phi_{I}^{I N V}}{\phi_{I}^{I N V} \sigma+\gamma_{I}^{I N V}+\psi_{I}\left(1-\phi_{I}^{I N V}\right)} & \Gamma_{\lambda, \lambda}^{I N V}=\frac{\partial \dot{\lambda}}{\partial \lambda}=\frac{(\rho+\delta)\left(\phi_{I}^{I N V}-\left(1-v\left(1-s_{I}\right)\right)\right)+\rho \psi_{I}\left(1-\phi_{I}^{I N V}\right)}{\phi_{I}^{I N V} \sigma+\gamma_{I}^{I N V}+\psi_{I}\left(1-\phi_{I}^{I N V}\right)} \\
\tau_{i, a}^{I N V}=\frac{\partial i}{\partial a}=\frac{(1-\alpha)\left(\gamma_{I}^{I N V}+\sigma\right)}{\phi_{I}^{I N V} \sigma+\gamma_{I}^{I N V}+\psi_{I}\left(1-\phi_{I}^{I N V}\right)} & b_{k, a}^{I N V}=\frac{\partial \dot{k}}{\partial a}=\frac{\delta\left(\gamma_{I}^{I N V}+\sigma\right)(1-\alpha)}{\phi_{I}^{I N V} \sigma+\gamma_{I}^{I N V}+\psi_{I}\left(1-\phi_{I}^{I N V}\right)} \\
\tau_{n, k}^{I N V}=\frac{\partial n}{\partial k}=\frac{v s_{I}\left(\alpha \sigma+\psi_{I}(1-\alpha)\right)}{\phi_{I}^{I N V} \sigma+\gamma_{I}^{I N V}+\psi_{I}\left(1-\phi_{I}^{I N V}\right)} & b_{\lambda, a}^{I N V}=\frac{\partial \dot{\lambda}}{\partial a}=\frac{(\rho+\delta)(1-\alpha)\left(\sigma\left(v\left(1-s_{I}\right)-1\right)-\gamma_{I}^{I N V}\right)}{\phi_{I}^{I N V} \sigma+\gamma_{I}^{I N V}+\psi_{I}\left(1-\phi_{I}^{I N V}\right)} \\
\tau_{n, \lambda}^{I N V}=\frac{\partial n}{\partial \lambda}=\frac{v s_{I}}{\phi_{I}^{I N V} \sigma+\gamma_{I}^{I N V}+\psi_{I}\left(1-\phi_{I}^{I N V}\right)} & +\frac{\psi_{I}(1-\alpha)\left[\rho\left(\sigma+\gamma_{I}^{I N V}\right)-(\rho+\delta) v\left(1-S_{I}\right)\right]}{\phi_{I}^{I N V} \sigma+\gamma_{I}^{I N V}+\psi_{I}\left(1-\phi_{I}^{I N V}\right)} \\
\frac{v s_{I}(1-\alpha)\left(\sigma-\psi_{I}\right)}{\phi_{I}^{I N V} \sigma+\gamma_{I}^{I N V}+\psi_{I}\left(1-\phi_{I}^{I N V}\right)} &
\end{array}
$$

Recall: $s_{I}=\frac{\alpha \delta}{\rho+\delta}, \phi_{I}^{I N V}=(1-(1-\alpha) v) s_{I}$, and $\gamma_{I}^{I N V}=(v-1)-\frac{v(1-\alpha)(1-\sigma) s_{I}}{\left(1-s_{I}\right)}$

\section{B.4.4 The Dynamic System}

The general solution to the dynamic system remains the same as before, but now with different coefficient values for $\tau_{x, x}^{I N V}$ 's, $\Gamma_{x, x}^{I N V}$ 's and $b_{x, x}$ 's. The new values for $\tau_{x, x}^{I N V}$ 's, $\Gamma_{x, x}^{I N V}$ 's and $b_{x, x}$ 's are given on the previous page. The solution to the dynamic system is:

$$
\lambda(0)=-\left[\frac{\Gamma_{k, \lambda}^{I N V} b_{\lambda, a}^{I N V}+\left(\mu_{2}^{I N V}-\Gamma_{\lambda, \lambda}^{I N V}\right) b_{k, a}^{I N V}}{\Gamma_{k, \lambda}^{I N V} \mu_{2}^{I N V}}\right] e^{-\mu_{2}^{I N V} T}
$$




$$
k(t)=\left\{\begin{array}{cc}
\frac{\Gamma_{k, \lambda}^{I N V} \lambda(0)}{\mu_{1}^{I N V}-\mu_{2}^{I N V}} e^{\mu_{1}^{I N V} t}+\frac{\Gamma_{k, \lambda}^{I N V} \lambda(0)}{\mu_{2}^{I N V}-\mu_{1}^{I N V}} e^{\mu_{2}^{I N V} t} & \text { for } t \in[0, T) \\
\frac{\Gamma_{k, \lambda}^{I N V} \lambda(0)}{\mu_{1-}^{I N V} \mu_{2}^{I N V}} e^{\mu_{1}^{I N V} t}+\frac{\Gamma_{k, \lambda}^{I N V} b_{\lambda, a}^{I N V}-\Gamma_{\lambda, \lambda}^{I N V} b_{k, a}^{I N V}}{\mu_{1}^{I N V} \mu_{2}^{I N V}}+\frac{\Gamma_{k, \lambda}^{I N V} b_{\lambda, a}^{I N V}+\left(\mu_{1}^{I N V}-\Gamma_{\lambda, \lambda}^{I N V}\right) b_{k, a}^{I N V}}{\mu_{1}^{I N V}\left(\mu_{1}^{I N V}-\mu_{2}^{I N V}\right)} e^{\mu_{1}^{I N V}(t-T)} & t \geq T
\end{array}\right.
$$

$$
\lambda(t)=\left\{\begin{array}{c}
\frac{\left(\mu_{1}^{I N V}-\Gamma_{k, k}^{I N V}\right) \lambda(0)}{\mu_{1}^{I N V}-\mu_{2}^{I N V}} e^{\mu_{1}^{I N V} t}+\frac{\left(\mu_{2}^{I N V}-\Gamma_{k, k}^{I N V}\right) \lambda(0)}{\mu_{2}^{I N V}-\mu_{1}^{I N V}} e^{\mu_{2}^{I N V} t} \\
\frac{\left(\mu_{1}^{I N V}-\Gamma_{k, k}^{I N V}\right) \lambda(0)}{\mu_{1}^{I N V}-\mu_{2}^{I N V}} e^{\mu_{1}^{I N V} t}+\frac{\Gamma_{\lambda, k}^{I N V} b_{k, a}^{I N V}-\Gamma_{k, k}^{I N V} b_{\lambda, a}^{I N V}}{\mu_{1}^{I N V} \mu_{2}^{I N V}}+\frac{\Gamma_{\lambda, k}^{I N V} b_{k, a}^{I N V}+\left(\mu_{1}^{I N V}-\Gamma_{k, k}^{I N V}\right) b_{\lambda, a}^{I N V}}{\mu_{1}^{I N V}\left(\mu_{1}^{I N V}-\mu_{2}^{I N V}\right)} e^{\mu_{1}^{I N V}(t-T)}
\end{array}\right.
$$$$
t \geq T
$$

Equations (135), (136), (137), (139), and (140), along with equation (138) give us a stable solution to our model economy for a $1 \%$ technology shock that occurs in period $T$.

\section{B.4.5 Proofs \& Expressions}

In this section for each lemma 1 - 5 and theorem 1 for the basic model with production complementarities we will either prove that the lemma or theorem for the basic model with production complementarities also holds for a model with investment adjustment costs, or we will present and prove an analagous lemma or theorem for a model with investment adjustment costs.

Lemma B.11: Lemma 1 from our analysis of the basic model also holds for a model with investment adjustment costs.

Proof. The consumption-investment production frontier given by equation (133) is identical to its counterpart in the basic model. The proof of lemma 1 depends purely on the consumption-investment production frontier equation. As a result Lemma 1 from the original model holds.

Lemma B.12: Lemma 2 from our analysis of the basic model also holds for a model with investment adjustment costs.

Proof. Given that the consumption-investment production frontier is identical to the basic model, to prove that lemma 2 holds for the investment adjustment cost model we only need 
to show that $c(0)>0$ if $\lambda(0)>0$.

Substituting (133) into (134) and solving for $c$ at time 0 leads to:

$$
c(0)=\frac{-\phi_{I}^{I N V}}{\gamma_{I}^{I N V}+\phi_{I}^{I N V} \sigma+\psi_{I}\left(1-\phi_{I}^{I N V}\right)} \lambda(0)
$$

If $v(1-\alpha)>1$ then $-\phi_{I}^{I N V}>0$ and $\gamma_{I}^{I N V}+\phi_{I}^{I N V} \sigma>0{ }^{44}$. Also, by assumption $\psi_{I}>0$. As a result from equation (141) if $\lambda(0)>0$ then $c(0)$ will increase.

Lemma B.13: Lemma 3 from our analysis of the basic model also holds for a model with investment adjustment costs.

Proof. To prove lemma 3 still holds we need to prove that the new $\tau_{c, k}^{I N V}, \tau_{c, \lambda}^{I N V} \tau_{i, k}^{I N V}, \tau_{i, \lambda}^{I N V}, \tau_{n, k}^{I N V}$, and $\tau_{n, \lambda}^{I N V}$ for the investment adjustment cost model are all positive. This follows trivially from the fact that if $v(1-\alpha)>1$ then $-\phi_{I}^{I N V}>0, \gamma_{I}^{I N V}>0$, and $\gamma_{I}^{I N V}+\phi_{I}^{I N V} \sigma>0{ }^{45}$, and by assumption $\psi_{I}>0$.

Lemma B.14: Lemma 4 from our analysis of the basic model also holds for a model with investment adjustment costs.

Proof. Identical to the basic model the dynamic system for a model with investment adjustment costs can be written as equation (17), albeit with different expressions for $\Gamma_{x, x}^{I N V}$ 's and $b_{x, x}$ 's. As a result to prove lemma 4 still holds it suffices to show that the new $\Gamma_{k, \lambda}^{I N V}$ and $\Gamma_{\lambda, \lambda}^{I N V}$ are still positive. $\Gamma_{k, \lambda}^{I N V}>0$ and $\Gamma_{\lambda, \lambda}^{I N V}>0$ for a model with investment adjustment costs follows trivially from the fact that if $v(1-\alpha)>1$ then $-\phi_{I}^{I N V}>0, \gamma_{I}^{I N V}>0$, $\left(\phi_{I}^{I N V}-\left(1-v\left(1-S_{I}\right)\right)\right)>0$ and $\gamma_{I}^{I N V}+\phi_{I}^{I N V} \sigma>0$, and by assumption $\psi_{I}>0$.

Lemma 5 and theorem 1 now change to reflect how movements in $\psi_{I}$ cause $\lambda(0)$ to change.

Lemma B.15: Suppose the economy experiences a positive technology news shock. Also, assume that $v>v_{*}=(1-\alpha)^{-1} \cdot \lambda(0)>0$ if and only if $\psi_{I}>\psi_{I}^{I N V *}$ where $\psi_{I}^{I N V *}$ is given by the equality $\Gamma_{k, \lambda}^{I N V} b_{\lambda, a}+\left(\mu_{2}^{I N V}-\Gamma_{\lambda, \lambda}^{I N V}\right) b_{k, a}^{I N V}=0$.

\footnotetext{
${ }^{44}$ For the proof see Lemma B.3.

${ }^{45}$ For the proof see Lemma B.1 \& B.3.
} 
Proof. Recall $\mu_{2}^{I N V}>0$ and $\Gamma_{k, \lambda}^{I N V}>0$, with:

$$
\lambda_{0}=-\left[\frac{\Gamma_{k, \lambda}^{I N V} b_{\lambda, a}^{I N V}+\left(\mu_{2}^{I N V}-\Gamma_{\lambda, \lambda}^{I N V}\right) b_{k, a}^{I N V}}{\Gamma_{k, \lambda}^{I N V} \mu_{2}^{I N V}}\right] e^{-\mu_{2}^{I N V} T}
$$

As a result $\lambda(0)>0$ if and only if $\Gamma_{k, \lambda}^{I N V} b_{\lambda, a}+\left(\mu_{2}^{I N V}-\Gamma_{\lambda, \lambda}^{I N V}\right) b_{k, a}^{I N V}<0$.

Theorem B.1: The investment adjusment cost model exhbits procyclical technology news shocks if and only if $v>v_{*}$ and $\psi_{I}>\psi_{I}^{I N V *}$.

Proof of Theorem 1. $\Leftarrow$. If $v(1-\alpha)>1$ and $\Gamma_{k, \lambda}^{I N V} b_{\lambda, a}+\left(\mu_{2}^{I N V}-\Gamma_{\lambda, \lambda}^{I N V}\right) b_{k, a}^{I N V}<0$, then a technology news shock is procyclical. Lemmas B.12 and B.15 prove the procyclical comovement at $t=0$, while Lemmas B.13, B.14 and B.15 establish the procyclical comovement for $0<t<T$.

$\Rightarrow$. If $v(1-\alpha)<1$ or $\Gamma_{k, \lambda}^{I N V} b_{\lambda, a}+\left(\mu_{2}^{I N V}-\Gamma_{\lambda, \lambda}^{I N V}\right) b_{k, a}^{I N V}>0$, then a technology news shock is not procyclical. This follow trivially from Lemma B.12, as the procyclical comovement will not occur at time $t=0$ if either of the above conditions are not met.

Lemma B.16: One of the eigenvalues of the $\Gamma^{I N V}$ matrix is positive and other negative.

Proof. The product of the eigenvalues is given by the determinant of $\Gamma^{I N V}$. The determinant of $\Gamma^{I N V}$ matrix can be shown to be equal to

$$
\operatorname{det}\left(\Gamma^{I N V}\right)=\frac{-\delta(\rho+\delta)}{\left(\phi_{I}^{I N V} \sigma+\gamma_{I}^{I N V}+\psi_{I}\left(1-\phi_{I}^{I N V}\right)\right)}\left[v\left(1-s_{I}\right)(1-\alpha)\right]<0
$$

As the product of the eigenvalues is negative it follows that the eigenvalues have opposite signs. Further, it can be shown that $\operatorname{tr}\left(\Gamma^{I N V}\right)=\rho$.

\section{B.5 A Model with Greenwood-Hercowitz-Huffman Preferences}

\section{B.5.1 The Model Economy}

A social planner has the following preferences

$$
V=(1-\omega)^{-1} \int_{0}^{\infty} e^{-\rho t}\left[C-\xi \frac{N^{1+\psi}}{1+\psi}\right]^{1-\omega} d t
$$


over time paths for consumption $C$ and hours worked $N$. We assume this functional form for the utility to preserve balanced growth. Also, $\rho=1 / \beta-1>0, \omega \geq 0, \psi \geq 0$, and $\xi>0$, where $\beta$ is the subjective discount factor, $\omega$ is the inverse of the intertemporal elasticity of substitution, $\psi$ is the inverse of the Frisch elasticity of labor, and $\xi$ is a scale parameter on the disutility of labor.

The planner is subject to the following constraints:

$$
\begin{aligned}
F[C(t), I(t)] & =K(t)^{\alpha}(A(t) N(t))^{1-\alpha} \\
\dot{K}(t) & =I(t)-\delta K(t)
\end{aligned}
$$

Here $K, I$ and $A$ represent capital, investment and the level of technology. The path of technology and the initial capital stock are exogenous. The depreciation rate, $\delta$, and the elasticity of output with respect to capital, $\alpha$, both lie between zero and one.

Further, we assume:

$$
F(C, I) \equiv\left[\theta C^{v}+(1-\theta) I^{v}\right]^{1 / v}
$$

where $\theta \in(0,1)$ and $v \geq 1$. When $v=1$, the equation collapses to the standard neo-classical case, which has infinite substitutability between the two goods. As $v$ increases, the complementarity between the production of the two goods increases. If $v=\infty$, the production frontier takes a Leontief form.

Next, let us define the exogenous processes - the technology news shock. The planner again has perfect foresight, with

$$
A(t)=\left\{\begin{array}{ccrl}
\bar{A} & \text { for } t & \in[0, T) \\
\tilde{A}=1.01 \times \bar{A} & t & \geq T
\end{array}\right.
$$

For the contemporaneous improvements case $T=0$ in the above specification. 


\section{B.5.2 The Model Economy's First Order Conditions}

The social planner chooses $C, I, K$, and $N$ to maximize $V$ subject to (143) and (144) taking as given the initial condition $K(0)$ and time path of technology. We can express the problem as a current value Hamiltonian:

$$
H=(1-\omega)^{-1}\left[C-\xi \frac{N^{1+\psi}}{1+\psi}\right]^{1-\omega}+\Lambda(I-\delta K)+\Phi\left(K^{\alpha}(A N)^{1-\alpha}-F(C, I)\right)
$$

The first-order necessary conditions at an interior solution satisfy :

$$
\begin{gathered}
-\frac{V_{N}}{V_{C}}=(1-\alpha) \frac{F}{N}\left(F_{C}\right)^{-1} \\
\frac{F_{I}}{F_{C}} V_{C}=\Lambda \\
\frac{\dot{\Lambda}}{\Lambda}-\rho=\delta-\alpha \frac{F}{K} \frac{V_{C}}{\Lambda F_{C}}
\end{gathered}
$$

along with our initial condition on capital and a transversality condition on $\Lambda$.

Equation (145) is the intratemporal Euler equation between consumption and labor hours, equation (146) is the intratemporal Euler equation between consumption and investment, and equation (147) is the optimal capital accumulation equation.

\section{B.5.3 The Model Economy Log Linearized and Simplified}

Given the first order conditions in the previous section our model economy can be described by the following five log linearized equations:

$$
\begin{gathered}
\left(1-s_{I}\right) c+s_{I} i=\alpha k+(1-\alpha)(a+n) \\
(1+\psi) n=v s_{I}(i-c)+c \\
\lambda=(v-1)(i-c)+\frac{1}{1-\frac{s_{n}}{1+\psi}}\left[-\omega c+\omega s_{n} n\right]
\end{gathered}
$$




$$
\begin{gathered}
\dot{k}=\delta(i-k) \\
\dot{\lambda}=-(\rho+\delta)\left[v\left(1-s_{I}\right)(c-i)+i-k\right]
\end{gathered}
$$

Here, $s_{I}=\frac{\alpha \delta}{\rho+\delta}$ and $s_{n}=\frac{1-\alpha}{1-S_{I}}$.

We can substitute (149) into (148) to get the consumption-investment production frontier ( $L_{1}$ line):

$$
\left(1-\frac{1-\alpha}{1+\psi}-\phi_{I}^{G H H}\right) c+\phi_{I}^{G H H} i=\alpha k+(1-\alpha) a
$$

Here, $\phi_{I}^{G H H}=\left(1-\frac{1-\alpha}{1+\psi} v\right) s_{I}$

We can also substitute (149) into (150) to get the consumption-investment euler equation ( $L_{2}$ line):

$$
\gamma_{I}^{G H H} i-\left(\omega+\gamma_{I}^{G H H}\right) c=\lambda
$$

Here, $\gamma_{I}^{G H H}=(v-1)+\frac{\omega v s_{I}}{\frac{1+v}{s_{n}}-1}$.

Equations (153) and (154) now give us a system of equations in $i$ and $c$ (treating $\lambda, k$, and $a$ as exogenous).

We also solve the system of equations above for $c, i, n, \dot{k}$, and $\dot{\lambda}$, assuming as given the state variable $\lambda$ and $k$, and the exogenous variable $a$ :

$$
\begin{aligned}
& c=\tau_{c, k}^{G H H} k+\tau_{c, \lambda}^{G H H} \lambda+\tau_{c, a}^{G H H} a \\
& i=\tau_{i, k}^{G H H} k+\tau_{i, \lambda}^{G H H} \lambda+\tau_{i, a}^{G H H} a \\
& n=\tau_{n, k}^{G H H} k+\tau_{n, \lambda}^{G H H} \lambda+\tau_{n, a}^{G H H} a \\
& \dot{k}=\Gamma_{k, k}^{G H H} k+\Gamma_{k, \lambda}^{G H H} \lambda+b_{k, a}^{G H H} a
\end{aligned}
$$




$$
\dot{\lambda}=\Gamma_{\lambda, k}^{G H H} k+\Gamma_{\lambda, \lambda}^{G H H} \lambda+b_{\lambda, a}^{G H H} a
$$

where,

$$
\begin{aligned}
\tau_{c, k}^{G H H}=\frac{\partial c}{\partial k}=\frac{\alpha \gamma_{I}^{G H H}}{\phi_{I}^{G H H} \omega+\left(1-\frac{1-\alpha}{1+\psi}\right) \gamma_{I}^{G H H}} & \Gamma_{k, k}^{G H H}=\frac{\partial \dot{k}}{\partial k}=\frac{-\delta\left(\left(1-\alpha-\frac{1-\alpha}{1+\psi}\right) \gamma_{I}^{G H H}+\phi_{I}^{G H H} \omega-\alpha \omega\right)}{\phi_{I}^{G H H} \omega+\left(1-\frac{1-\alpha}{1+\psi}\right) \gamma_{I}^{G H H}} \\
\tau_{c, \lambda}^{G H H}=\frac{\partial c}{\partial k}=-\frac{\phi_{I}^{G H H}}{\phi_{I}^{G H H} \omega+\left(1-\frac{1-\alpha}{1+\psi}\right) \gamma_{I}^{G H H}} & \Gamma_{k, \lambda}^{G H H}=\frac{\partial \dot{k}}{\partial \lambda}=\frac{\delta\left(1-\frac{1-\alpha}{1+\psi}-\phi_{I}^{G H H}\right)}{\phi_{I}^{G H H} \omega+\left(1-\frac{1-\alpha}{1+\psi}\right) \gamma_{I}^{G H H}} \\
\tau_{c, a}^{G H H}=\frac{\partial c}{\partial a}=\frac{(1-\alpha) \gamma_{I}^{G H H}}{\phi_{I}^{G H H} \omega+\left(1-\frac{1-\alpha}{1+\psi}\right) \gamma_{I}^{G H H}} & \Gamma_{\lambda, k}^{G H H}=\frac{\partial \dot{\lambda}}{\partial k}=\frac{(\rho+\delta)\left(\left(1-\alpha-\frac{1-\alpha}{1+\psi}\right) \gamma_{I}^{G H H}+\phi_{I}^{G H H} \omega-\alpha \omega+\alpha \sigma v\left(1-s_{I}\right)\right)}{\phi_{I}^{G H H} \omega+\left(1-\frac{1-\alpha}{1+\psi}\right) \gamma_{I}^{G H H}} \\
\tau_{i, k}^{G H H}=\frac{\partial i}{\partial k}=\frac{\alpha\left(\gamma_{I}^{G H H}+\omega\right)}{\phi_{I}^{G H H} \omega+\left(1-\frac{1-\alpha}{1+\psi}\right) \gamma_{I}^{G H H}} & \Gamma_{\lambda, \lambda}^{G H H}=\frac{\partial \dot{\lambda}}{\partial \lambda}=\frac{(\rho+\delta)\left(\phi_{I}^{G H H}-\left(1-\frac{1-\alpha}{1+\psi}\right)\left(1-v\left(1-s_{I}\right)\right)\right)}{\phi_{I}^{G H H} \omega+\left(1-\frac{1-\alpha}{1+\psi}\right) \gamma_{I}^{G H H}} \\
\tau_{i, \lambda}^{G H H}=\frac{\partial i}{\partial \lambda}=\frac{\left(1-\frac{1-\alpha}{1+\psi}\right)-\phi_{I}^{G H H}}{\phi_{I}^{G H H} \omega+\left(1-\frac{1-\alpha}{1+\psi}\right) \gamma_{I}^{G H H}} & b_{k, a}^{G H H}=\frac{\partial \dot{k}}{\partial a}=\frac{\delta(1-\alpha)\left(\omega+\gamma_{I}^{G H H}\right)}{\phi_{I}^{G H H} \omega+\left(1-\frac{1-\alpha}{1+\psi}\right) \gamma_{I}^{G H H}} \\
\tau_{i, a}^{G H H}=\frac{\partial i}{\partial a}=\frac{(1-\alpha)\left(\omega+\gamma_{I}^{G H H}\right)}{\phi_{I}^{G H H} \omega+\left(1-\frac{1-\alpha}{1+\psi}\right) \gamma_{I}^{G H H}} & b_{\lambda, a}^{G H H}=\frac{\partial \dot{\lambda}}{\partial a}=\frac{(\rho+\delta)(1-\alpha)\left(\omega\left(v\left(1-s_{I}\right)-1\right)-\gamma_{I}^{G H H}\right)}{\phi_{I}^{G H H} \omega+\left(1-\frac{1-\alpha}{1+\psi}\right) \gamma_{I}^{G H H}} \\
\tau_{n, k}^{G H H}=\frac{\partial n}{\partial k}=\frac{\alpha\left(\gamma_{I}^{G H H}+\nu s_{I} \omega\right)}{(1+\psi)\left(\phi_{I}^{G H H} \omega+\left(1-\frac{1-\alpha}{1+\psi}\right) \gamma_{I}^{G H H}\right)} & \\
\tau_{n, \lambda}^{G H H}=\frac{\partial n}{\partial \lambda}=\frac{v s_{I}\left(1-\frac{1-\alpha}{1+\psi}\right)-\phi_{I}^{G H H}}{(1+\psi)\left(\phi_{I}^{G H H} \omega+\left(1-\frac{1-\alpha}{1+\psi}\right) \gamma_{I}^{G H H}\right)} & \\
\tau_{n, a}^{G H H}=\frac{\partial n}{\partial a}=\frac{(1-\alpha)\left(\gamma_{I}^{G H H}+\nu s_{I} \omega\right)}{(1+\psi)\left(\phi_{I}^{G H H} \omega+\left(1-\frac{1-\alpha}{1+\psi}\right) \gamma_{I}^{G H H}\right)} &
\end{aligned}
$$

Recall: $s_{I}=\frac{\alpha \delta}{\rho+\delta}, s_{n}=\frac{1-\alpha}{1-S_{I}}, \phi_{I}^{G H H}=\left(1-\frac{1-\alpha}{1+\psi} v\right) s_{I}$, and $\gamma_{I}^{G H H}=(v-1)+\frac{\omega v s_{I}}{\frac{1+\psi}{s_{n}}-1}$

\section{B.5.4 The Dynamic System}

The general solution to the dynamic system remains the same as before, but now with different coefficient values for $\tau_{x, x}^{G H H}$ 's, $\Gamma_{x, x}^{G H H}$ 's and $b_{x, x}^{G H H}$ 's. The new values for $\tau_{x, x}^{G H H}$ 's, $\Gamma_{x, x}^{G H H}$ 's and $b_{x, x}^{G H H}$ 's are given on the previous page. The solution to the dynamic system is:

$$
\lambda(0)=-\left[\frac{\Gamma_{k, \lambda}^{G H H} b_{\lambda, a}^{G H H}+\left(\mu_{2}^{G H H}-\Gamma_{\lambda, \lambda}^{G H H}\right) b_{k, a}^{G H H}}{\Gamma_{k, \lambda}^{G H H} \mu_{2}^{G H H}}\right] e^{-\mu_{2}^{G H H} T}
$$




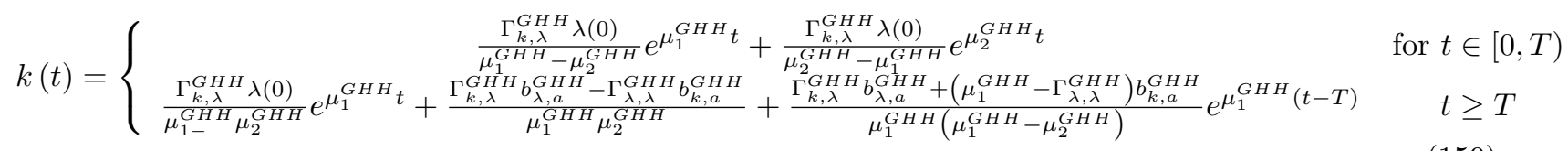

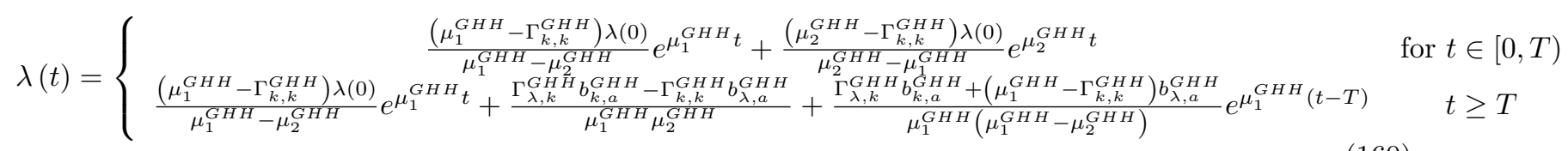

Equations (155), (156), (157), (159), and (160), along with equation (158) give us a stable solution to our model economy for a $1 \%$ technology shock that occurs in period $T$.

\section{B.5.5 Proofs \& Expressions}

In this section for each lemma 1 - 5 and theorem 1 for the basic model with production complementarities we will either prove that the lemma or theorem for the basic model with production complementarities also holds for a model with GHH preferences, or we will present and prove an analagous lemma or theorem for a model with $\mathrm{GHH}$ for $\psi \rightarrow 0$.

Lemma B.17: Lemma 1 from our analysis of the basic model also holds for a model with GHH Preferences.

Proof. The consumption-investment production frontier is given by equation (153). For $\psi \rightarrow$ $0 \phi_{I}^{P C}=\phi_{I}^{G H H}$, and thus for $v(1-\alpha)>1$ we have $\phi_{I}^{G H H}<0$ and $\left(\left(1-\frac{1-\alpha}{1+\psi}\right)-\phi_{I}^{G H H}\right)>0$ ${ }^{46}$.As a result Lemma 1 from the original model holds and the proof follows from the proof of Lemma 1.

Lemma B.18: Lemma 2 from our analysis of the basic model also holds for a model with GHH Preferences with the added condition that $\omega<\frac{1}{\delta}$.

$$
46\left(1-\frac{1-\alpha}{1+\psi}\right)=\frac{\psi+\alpha}{1+\psi}>0
$$


Proof. Given that the consumption-investment production frontier is upward sloping to prove that lemma 2 holds for the GHH model we only need to show that $c(0)>0$ if $\lambda(0)>0$.

Substituting (153) into (154) and solving for $c$ at time 0 leads to:

$$
c(0)=\frac{-\phi_{I}^{G H H}}{\left(1-\frac{1-\alpha}{1+\psi}\right) \gamma_{I}^{G H H}+\phi_{I}^{G H H} \omega} \lambda(0)
$$

If $v(1-\alpha)>1$ then $-\phi_{I}^{G H H}>0$ and for $\omega<\frac{1}{\delta}$ we also have $\left(1-\frac{1-\alpha}{1+\psi}\right) \gamma_{I}^{G H H}+\phi_{I}^{G H H} \omega>0$. As a result from equation (161) if $\lambda(0)>0$ then $c(0)$ will increase.

Lemma B.19: Lemma 3 from our analysis of the basic model also holds for a model with GHH Preferences.

Proof. To prove lemma 3 still holds we need to prove that the new $\tau_{c, k}^{G H H}, \tau_{c, \lambda}^{G H H} \tau_{i, k}^{G H H}, \tau_{i, \lambda}^{G H H}, \tau_{n, k}^{G H H}$, and $\tau_{n, \lambda}^{G H H}$ for the GHH preferences model are all positive. This follows trivially from the fact that $\left(1-\frac{1-\alpha}{1+\psi}\right)>0$, if $v(1-\alpha)>1$ then $-\phi_{I}^{G H H}>0, \gamma_{I}^{G H H}>0$, additionally for $\omega<\frac{1}{\delta}$ we also have $\left(1-\frac{1-\alpha}{1+\psi}\right) \gamma_{I}^{G H H}+\phi_{I}^{G H H} \omega>0$.

Lemma B.20: Lemma 4 from our analysis of the basic model also holds for a model with GHH Preferences.

Proof. Identical to the basic model the dynamic system for a model with GHH preferences can be written as equation (17), albeit with different expressions for $\Gamma_{x, x}^{G H H}$ 's and $b_{x, x}^{G H H}$ 's. As a result to prove lemma 4 still holds it suffices to show that the new $\Gamma_{k, \lambda}^{G H H}$ and $\Gamma_{\lambda, \lambda}^{G H H}$ are still positive. $\Gamma_{k, \lambda}^{G H H}>0$ and $\Gamma_{\lambda, \lambda}^{G H H}>0$ for a model with GHH prefences follows trivially from the fact that $\left(1-\frac{1-\alpha}{1+\psi}\right)>0$, if $v(1-\alpha)>1$ then $-\phi_{I}^{G H H}>0, \gamma_{I}^{G H H}>0$, and $\left(\phi_{I}^{G H H}-\left(1-\frac{1-\alpha}{1+\psi}\right)\left(1-v\left(1-S_{I}\right)\right)\right)>0$.

Lemma 5 and theorem 1 now change to reflect how movements in $\omega$ cause $\lambda(0)$ to change.

Lemma B.21: Suppose the economy experiences a positive technology news shock. Also, assume that $v>v_{*}=(1-\alpha)^{-1}$. $\lambda(0)>0$ if and only if $\omega>\omega^{*}$ where $\omega^{*}$ is given by the equality $\Gamma_{k, \lambda}^{G H H} b_{\lambda, a}^{G H H}+\left(\mu_{2}^{G H H}-\Gamma_{\lambda, \lambda}^{G H H}\right) b_{k, a}^{G H H}=0$. 
Proof. Recall $\mu_{2}^{G H H}>0$ and $\Gamma_{k, \lambda}^{G H H}>0$, with:

$$
\lambda_{0}=-\left[\frac{\Gamma_{k, \lambda}^{G H H} b_{\lambda, a}^{G H H}+\left(\mu_{2}^{G H H}-\Gamma_{\lambda, \lambda}^{G H H}\right) b_{k, a}^{G H H}}{\Gamma_{k, \lambda}^{G H H} \mu_{2}^{G H H}}\right] e^{-\mu_{2}^{G H H} T}
$$

As a result $\lambda(0)>0$ if and only if $\Gamma_{k, \lambda}^{G H H} b_{\lambda, a}+\left(\mu_{2}^{G H H}-\Gamma_{\lambda, \lambda}^{G H H}\right) b_{k, a}^{G H H}<0$.

Theorem B.2: The GHH Preference model exhbits procyclical technology news shocks if and only if $v>v_{*}$ and $\omega>\omega^{*}$.

Proof of Theorem 1. $\Leftarrow$. If $v(1-\alpha)>1$ and $\Gamma_{k, \lambda}^{G H H} b_{\lambda, a}+\left(\mu_{2}^{G H H}-\Gamma_{\lambda, \lambda}^{G H H}\right) b_{k, a}^{G H H}<0$, then a technology news shock is procyclical. Lemmas B.17 and B.18 prove the procyclical comovement at $t=0$, while Lemmas B.19-B.21 establish the procyclical comovement for $0<t<T$.

$\Rightarrow$. If $v(1-\alpha)<1$ or $\Gamma_{k, \lambda}^{G H H} b_{\lambda, a}+\left(\mu_{2}^{G H H}-\Gamma_{\lambda, \lambda}^{G H H}\right) b_{k, a}^{G H H}>0$, then a technology news shock is not procyclical. This follow trivially from Lemma B.17, as the procyclical comovement will not occur at time $t=0$ if either of the above conditions are not met.

Lemma B.22: One of the eigenvalues of the $\Gamma^{G H H}$ matrix is positive and other negative.

Proof. The product of the eigenvalues is given by the determinant of $\Gamma^{G H H}$. The determinant of $\Gamma^{G H H}$ matrix can be shown to be equal to

$$
\operatorname{det}\left(\Gamma^{G H H}\right)=\frac{-\delta(\rho+\delta)}{(1+\psi)\left(\phi_{I}^{G H H} \omega+\left(1-\frac{1-\alpha}{1+\psi}\right) \gamma_{I}^{G H H}\right)}\left[\psi v\left(1-s_{I}\right)(1-\alpha)\right]<0
$$

As the product of the eigenvalues is negative it follows that the eigenvalues have opposite signs. Further, it can be shown that $\operatorname{tr}\left(\Gamma^{G H H}\right)=\rho$. 